TITLE:

\title{
Theoretical study of the hydrogen adsorption on AlB nanowire
}

\section{$\operatorname{AUTHOR}(\mathrm{S})$ :}

Fukushima, Akinori; Doi, Kentaro; Senami, Masato; Tachibana, Akitomo

\section{CITATION:}

Fukushima, Akinori ...[et al]. Theoretical study of the hydrogen

adsorption on AlB nanowire. Journal of Power Sources 2008, 184(1): 6076

\section{ISSUE DATE:}

2008-09-15

URL:

http://hdl.handle.net/2433/89658

\section{RIGHT:}

Copyright (c) 2008 Elsevier B.V.; This is not the published version. Please cite only the published version.; この論文は出版社版でありません。引 用の際には出版社版をご確認ご利用ください。 


\title{
Theoretical study of the hydrogen adsorption
}

\section{on AlB nanowire}

Akinori Fukushima, Kentaro Doi, Masato Senami and Akitomo Tachibana*

Department of Micro Engineering, Kyoto University, Kyoto 606-8501, Japan

${ }^{*}$ Corresponding author, phone \& fax: $+81-75-753-5184$

*Electronic address: akitomo@scl.kyoto-u.ac.jp

\begin{abstract}
We studied AlB nanowires as hydrogen storage materials based on density functional theory and Rigged QED theory. In this paper, we focused on the adsorption energy and the electronic stucture of models. AlB nanowire models are compared with an $\mathrm{Al}$ nanowire model and $\mathrm{AlB}_{2}$ crystal structure in terms of density of states, electron density, kinetic energy density, tension density and stress tensor density. These results revealed AlB nanowires do not have the conductivity, while the $\mathrm{Al}$ nanowire and $\mathrm{AlB}_{2}$ bulk have it. It was also shown that the stabilization energies of AlB nanowires for the hydrogen adsorption are larger than that of $\mathrm{Al}$ nanowire. Adsorped hydrogens are more stable in the AlB nanowires than the $\mathrm{Al}$ nanowire. Key words: First-principle calculation;Hydrogen adsorption;Aluminum nanowire; Quantum energy density
\end{abstract}




\section{Introduction}

Nowadays, hydrogen energy is focused on as a clean energy resource, taking the place of fossil fuel. However, hydrogen is gas in room temperature and 1 atm, and thus hydrogen is difficult to store safely. One of methods of hydrogen storage is using hydrogen adsorption. For the hydrogen adsorption, higher weight percent storage is required for hydrogen to take rank with fossil fuel. However, high-weight-percent hydrogen storage has not been achieved for most of hydrogen storage materials. To overcome the difficulty, nanostructures are focused on. Nanostructures have larger surfaces than usual bulk structures. In this point, they have a possibility to store much hydrogen. Thus many studies are reported about hydrogen storage nanostructures[1-3].

In nano materials, we particularly focused on one-dimensional structures such as a carbon nanotube $(\mathrm{CNT})$ and $\mathrm{Al}$ nanowire[1,4-5]. Makita et al.[4] calculated optimized geometries of aluminum nanowires based on gold nanowires using first-principles electronic structure calculations. Kawakami et al.[5] showed that hydrogen is adsorbed on a pentagonal $\mathrm{Al}$ nanowire model by the separation of a $\mathrm{H}_{2}$ molecule into two $\mathrm{H}$ atoms. Nakano et al.[1] suggested to wrap $\mathrm{Al}$ species in carbon materials to enhance the hydrogen adsorption on CNT. Note that Suzuki et al.[6] reported that Al whiskers are actually fabricated by glancing angle deposition on a high temperature (HT-GLAD) substrate. For a hydrogen molecule adsorption on these hydrogen storage materials, the low adsorption and dissociation energy under $1.0 \mathrm{eV}$ is for the usage as a 
fuel cell. However, these energy are generically larger than $1.0 \mathrm{eV}$ for these nanostructures. As a solution strategy to overcome the high adsorption and dissorption energy, we suggest the use of the electronic and magnetic force. Thus in this paper, we focus on hydrogen adsorption abilities and the electronic structures of the $\mathrm{AlB}$ and $\mathrm{Al}$ nanowires. AlB nanowires are expected to be a better candidate for hydrogen storage because of light weight of $\mathrm{B}$ comparing to Al. We carry out first-principles calculations to optimized geometries, analyze electronic structures and clarify the influence of substitution of $\mathrm{Al}$ with $\mathrm{B}$ for the electronic structures. In addition, we analyze details of the electronic structures in terms of the quantum mechanical energy densities based on the regional density functional theory[7-13].

\section{Computational Methods}

\subsection{Calculation model and method}

Two AlB nanowire models for our calculation are shown in Figs. 1 and 2 . Figure 1(a) shows $\mathrm{AlB}_{2}$ bulk models[14]. Figures 1(b) and 1(c) shows the hexagonal nanowire models based on $\mathrm{AlB}_{2}$ bulk models. Figure 2(1) and 1(e) are $\mathrm{AlB}_{2}$ bulk surface models, which are called as the surface model 1 and model 2 respectively. These models are also based on $\mathrm{AlB}_{2}$ bulk models. In this paper, the small nanowire of Fig. 1(c) is considered to be a hydrogen storage material. Other models are used to compare with nanowire models. 
In Fig. 2, another $\mathrm{AlB}$ nanowire model and $\mathrm{Al}$ nanowire are shown. This $\mathrm{AlB}$ nanowire model is composed of pentagonal $\mathrm{B}$ rings and an $\mathrm{Al}$ monoatomic wire. Figure 2(a) shows the $\mathrm{Al}$ nanowire whose geometry was calculated by Makita et $a .^{2}$ consisting of pentagonal rings and monoatomic wire. Pentagonal $\mathrm{B}$ rings are placed with rotating by 36 degrees for each ring. The AlB pentagonal nanowire shown in Fig. 2(b) is based on this Al nanowire. This AlB nanowire is also cinsidered to be a hydrogen storage material.

The boundary condition of these models is imposed as periodic one. In our calculation, two kinds of supercell are used. $8.36 \AA \times 8.36 \AA \times 4 \mathrm{c} \AA$ supercell is considered for AlB nanowires and $10.6 \AA \times 10.6 \AA \times 8 \mathrm{c} \AA$ supercell is considered for $\mathrm{Al}$ nanowire. The parameter $c$ is the distance from atoms on the axis to the pentagonal or hexagonal ring. If the smaller unit cell is used for $\mathrm{Al}$ nanowire, the interaction with next unit cell is not negligible. The supercells for $\mathrm{AlB}_{2}$ surface models shown in Fig. $1(\mathrm{~d})$ and $1(\mathrm{e})$ are $5.19 \AA \times 6.54 \AA \times$ $10.4 \AA$ and $5.99 \AA \times 6.54 \AA \times 10.4 \AA$, respectively.

To investigate the electronic structures of the systems, variational calculations are carried out based on density functional theory, which is implemented by FHI98md program package[15]. Wave functions of valence electrons are expanded by Bloch functions, while atomic cores and inner-shell electrons are replaced by Hamman's norm conserving pseudopotentials[16]. The generalizedgradient-approximation method is employed for exchange-correlation interaction formulated by Pardew and Wang (PW91)[17]. The values of cutoff energy for wave functions with plane-wave expansion are chosen as $1088 \mathrm{eV}$ for the 
$\mathrm{AlB}$ nanowires and $408 \mathrm{eV}$ for the $\mathrm{Al}$ nanowire. The higher cutoff energy, 1088 $\mathrm{eV}$, is required to derive an accurate result for $\mathrm{B}$ atoms. The cutoff energy for bulk surface model is the same as the lower cutoff energy. This is because the vacuum region is only one direction. Thus the lower cutoff energy is sufficient. The density of states (DOS) are calculated using a density functional method with ADF program package[18]. Electron wave functions are expanded with linear combinations of atomic orbital basis set. In our calculation, hybridization of Slater type double-zeta basis and numerical one are employed.

The minimum point in a potential energy surface corresponds optimized structure. In the optimization calculation, two geometric parameters, the radius of nanowire $r_{1}$ and the length of the unit $c$, are determined. The geometries of our models are optimized with keeping their symmetries.

\subsection{Quantum energy density}

Quantum energy density[7-10] provides a number of information about electronic properties. First, we can derive kinetic energy density $n_{T}(\vec{r})$ from electron wave functions as follows,

$$
n_{T}(\vec{r})=-\frac{\hbar^{2}}{4 m} \sum_{i} \nu_{i}\left[\psi_{i}^{*}(\vec{r}) \triangle \psi_{i}(\vec{r})+\triangle \psi_{i}^{*}(\vec{r}) \psi_{i}(\vec{r})\right],
$$

where $\nu_{i}$ is the occupation number of the $i$ th state $\psi_{i}$. This energy density represents whether electrons can behave as classical particle. Electrons can 
freely move as obeying classical mechanics in positive kinetic energy density region, which is called as electronic drop region $R_{D}$. On the other hand, in the negative kinetic energy density region, which is called as electronic atmosphere region $R_{A}$, electrons cannot move freely and exist by only quantum tunneling effects. The boundary of these two regions is called electronic interface $S$. Stress tensor density $\overleftrightarrow{\tau}^{S}(\vec{r})$ is denoted as a $3 \times 3$ matrix whose element $\tau^{S k l}(\vec{r})$ is given by

$$
\begin{aligned}
\tau^{S k l}(\vec{r})=\frac{\hbar^{2}}{4 m} \sum_{i} & \nu_{i}\left[\psi_{i}^{*}(\vec{r}) \frac{\partial^{2} \psi_{i}(\vec{r})}{\partial x^{k} \partial x^{l}}-\frac{\partial \psi_{i}^{*}(\vec{r})}{\partial x^{k}} \frac{\partial \psi_{i}(\vec{r})}{\partial x^{l}}\right. \\
+ & \left.\frac{\partial^{2} \psi_{i}^{*}(\vec{r})}{\partial x^{k} \partial x^{l}} \psi_{i}(\vec{r})-\frac{\partial \psi_{i}^{*}(\vec{r})}{\partial x^{l}} \frac{\partial \psi_{i}(\vec{r})}{\partial x^{k}}\right] .
\end{aligned}
$$

The positive and negative eigenvalues of $\stackrel{\leftrightarrow}{\tau}^{S}(\vec{r})$ represent the tensile and compressive stress, respectively. This tensor clarifies properties of chemical bonds, i.e., covalent bonds and metallic ones are distinguished. In this paper, we show the largest eigenvalues and their eigenvectors as the principal stress.

Tension density $\vec{\tau}^{S}(\vec{r})=\left(\tau^{S 1}(\vec{r}), \tau^{S 2}(\vec{r}), \tau^{S 3}(\vec{r})\right)$ is given as the divergence of the stress tensor density, $\stackrel{\leftrightarrow}{\tau}^{S}(\vec{r})$,

$$
\begin{array}{r}
\tau^{S k}(\vec{r})=\frac{\hbar^{2}}{4 m} \sum_{i} \nu_{i}\left[\psi_{i}^{*}(\vec{r}) \frac{\partial \triangle \psi_{i}(\vec{r})}{\partial x^{k}}-\frac{\partial \psi_{i}^{*}(\vec{r})}{\partial x^{k}} \Delta \psi_{i}(\vec{r})\right. \\
\left.+\frac{\partial \triangle \psi_{i}^{*}(\vec{r})}{\partial x^{k}} \psi_{i}(\vec{r})-\triangle \psi_{i}^{*}(\vec{r}) \frac{\partial \psi_{i}(\vec{r})}{\partial x^{k}}\right]
\end{array}
$$




\section{Results and Discussion}

\subsection{Geometry of AlB nanowires}

Figure 3 shows the potential energy curves of both AlB nanowires for hydrogen storage. The energy curves are shown depending on the parameter $r_{1}$ and c. The optimized parameter corresponds to the minimum energy. The calculation is done in detail around the stable geometry. In the case of the pentagonal AlB nanowire, $r_{1}$ and $c$ are determined at $1.31 \AA$ and $1.80 \AA$, respectively. In this structure, the bond length between $\mathrm{B}$ atoms on the same ring is $1.54 \AA$ and that between $\mathrm{B}$ and $\mathrm{Al}$ atoms is $2.23 \AA$. In the hexagonal $\mathrm{AlB}$ nanowire, $r_{1}$ and $c$ are $1.54 \AA$ and $1.68 \AA$, respectively, and the length between B (B and $\mathrm{Al})$ atoms is $1.54 \AA(2.28 \AA)$. The optimized lengths of $\mathrm{B}-\mathrm{B}$ bond are the same for both models, while other parameters are different from each other.

On the other hand, in larger hexagonal nanowire models, the B-B bond length is $1.67 \AA$ and the $\mathrm{B}-\mathrm{Al}$ length is $2.36 \AA$. Moreover the interatomic distances in $\mathrm{AlB}_{2}$ crystal are known as $1.73 \AA$ for $\mathrm{B}-\mathrm{B}$ and $2.38 \AA$ for $\mathrm{B}-\mathrm{Al}$, these bond lengths are shorter in nanowire structures. In $\mathrm{Al}$ nanowire, the wire radius $r_{1}$ and the length of the unit $c$ are $2.48 \AA$ and $1.34 \AA$ respectively.

Figure 4 shows $\triangle E / \triangle c$ as a function of $\triangle c$, where $\triangle c$ is the difference between $c$ and that of the stable geometry, and $\triangle E$ is the difference in the potential energy. In both simple AlB nanowire models, the line can be approximately considered as straight. The gradients of the lines mean the stability 
of nanowires. This figure shows that larger energy is needed for larger $\triangle c$. Therefore, the gradients are regarded as Young's moduls. Thus these figures indicate the stabilization of simple AlB nanowires in the axial direction. But the geometry which is calculated with keeping symmetry may be a meta-stable one. The stability of nanowire model is investigated by the deformation along the normal vibrational modes, where the norm of the eigenvectors is set to 0.1 $\AA$. The normal vibrationl modes are calculated with Gaussian 03 in the molecular system[19]. Atoms of models are moved to the direction of the imaginary normal vibrational modes and the total energy are calculated with this models in the periodic system. As a result, these nanowires are stable enough.

\subsubsection{Electronic structure of simple nanowires}

First, we discuss the electronic structure of nanowire models. The DOS and band structure of simple nanowire models are shown in Fig. 5. The Fermi levels are shifted to the origin in the horizontal axes. In addition, the DOS of the $\mathrm{AlB}_{2}$ bulk, surface and larger nanowire models are shown in Fig. 6. Moreover the band structure of larger surface models are shown in Fig. 6. The Fermi levels are also shifted as figures of simple nanowire models. In the pentagonal AlB nanowire, the band gap on the Fermi level is seen in Fig. 5(a). Hence this indicates that AlB pentagonal nanowire has different conductive property from $\mathrm{AlB}_{2}$ bulk structure. On the other hands, DOS and band structure of AlB hexagonal nanowire in Fig. 5(b) is metallic. Moreover the DOS and 
band structure of $\mathrm{AlB}_{2}$ surface and larger hexagonal nanowire are shown in Figs. 6(a),(b),(c). In Al pentagonal nanowire model, DOS and band structure is metallic.

Next, to study electronic state of the simple nanowire models in detail, the electron density, the kinetic energy density, tension density and stress tensor density are calculated. The electron density on the $x z$-plane and $x y$-plane are shown in Fig. 7. Figures 7(a),(b),(d),(e),(g), and (h) are the sum of the occupied states and (c),(f) and (i) are the highest occupied state. Figures 7(a),(d), and $(\mathrm{g})$ show electron density on $x y$-plane and the other figures show that of $x z$-plane. In Fig. 8, the kinetic energy density and the tension density is shown. Figures $8(\mathrm{a}),(\mathrm{b}),(\mathrm{d}),(\mathrm{e}),(\mathrm{g})$, and $(\mathrm{h})$ are the sum of the occupied states and (c),(f) and (i) are the highest occupied state. The tension density is normalized. Figures $8(\mathrm{a}),(\mathrm{d})$, and $(\mathrm{g})$ show the kinetic energy density and tension density on $x y$-plane and the other figures show those of $x z$-plane. Figure 9 shows the largest eigenvalue and eigenvector of stress tensor density. The eigenvector is normalized. Figures 9(a),(c), and (e) show electron density on $x y$-plane and the other figures show that of $x z$-plane.

First, we discuss the pentagonal AlB nanowire. In Figs. 7(a) and (b), the electron density between B atoms is high. It shows that B atoms are bonded each other. It is seen from Fig. 7(c) that the electron density of the highest occupied state concentrates on $\mathrm{B}$ atoms rather than on $\mathrm{Al}$ atoms. The highest occupied state $\mathrm{B}(2 s 2 p)$ appears on the direction perpendicular to the wire axis like dangling bonds. Hence, it is seen that it makes bonds not along the 
wire axis but between $\mathrm{B}$ atoms on the five-membered ring. Therefore, in the pentagonal AlB nanowire, electrons cannot move along the nanowire axis like free electrons. Moreover, it can be speculated that this concentrated electron density affects $\mathrm{H}$ atom adsorption. This tendency is also seen in the kinetic energy density and tension density. In Figs. 8(a) and (b), the kinetic energy density concentrate between B atoms and in Fig. 8(c) the kinetic energy density of the highest occupied state concentrate on the direction perpendicular to the wire axis like dangling bonds. In Figs. 9(a) and (b), the largest eigenvalue and the eigenvector are shown. The red (blue) region means the positive (negative) eigenvalue of the stress tensor density. The positive stress is seen between B atoms and spindle-structure is seen in the same region. This means $\mathrm{B}-\mathrm{B}$ bond has the covalent property.

Properties of the hexagonal AlB nanowire are similar to those of the pentagonal one as seen in Figs. 7, 8 and 9. However, in the hexagonal one, the highest occupied state $\mathrm{B}(2 s 2 p)$ appears not only on the direction perpendicular to the wire axis. It appears around $\mathrm{B}$ atoms, which is in contrast with that of the pentagonal one. The interaction of these atomic orbitals is weak, and hence narrow band appears near the Fermi level as seen in Fig. 5(b). However, electrons on the narrow band are not conductive, since electrons concentrates on $\mathrm{B}(2 s 2 p)$ hybridized orbitals and heavy effective masses are understood from the DOS and band structure. Thus, the motion of electrons is also constrained only on the atoms, and hence this nanowire is expected not to have the conductivity as the pentagonal one. 
Next, we consider the pentagonal Al nanowire. The band structure shows metallic properties as shown in Fig. 5(c). In the $\mathrm{Al}$ nanowire, the electron density and the positive kinetic energy density region is observed in the whole $\mathrm{Al}$ nanowire (Figs. 7(g)-(i) and 8(g)-(i)). Hence, it is expected that electrons can be accelerated by the external fields and escape from the constraint of atomic nuclei. Therefore, it can be concluded that $\mathrm{Al}$ nanowire has conductivity. The largest eigenvalue is almost negative on the $x z$-plane and $x y$-plane. This shows metallic bond between $\mathrm{Al}$ atoms. In $\mathrm{Al}$ nanowire models, electrons of HOMO state are not localized. Thus it can be speculated that AlB nanowire has different hydrogen adsorption property.

\subsection{Hydrogen adsorption on AlB nanowires}

\subsubsection{Hydrogen adsorption site and energy}

In this section, hydrogen adsorption on the nanowires is discussed. Total energy is calculated to find out the stable adsorption point of a $\mathrm{H}$ atom on the nanowires. Three adsorption points, site 1, 2, and 3, as shown in Fig. 10 are considered. First, the geometries of the nanowires are fixed for the calculation of total energy. The stabilization energy $\triangle E$ is defined as

$$
\triangle E=E(\text { nanowire }+\mathrm{H})-\{E(\text { nanowire })+E(\mathrm{H})\} .
$$


The results of $\triangle E$ are shown in Table 1. In these results, the stabilization energy is the lowest on site 1 for all models. Therefore, a $\mathrm{H}$ atom is adsorbed on the top of $\mathrm{B}(\mathrm{Al})$ atoms. In the following, we only consider the hydrogen adsorption on the top of $\mathrm{Al}$ or $\mathrm{B}$ of the ring layer.

Next we consider the adsorption of $\mathrm{H}_{2}$ molecules. The number of $\mathrm{H}$ atoms is even, and therefore the following definition of the adsorption energy is used,

$$
\triangle E=\frac{E\left(\text { nanowire }+N \times \mathrm{H}_{2}\right)-\left\{E(\text { nanowire })+N \times E\left(\mathrm{H}_{2}\right)\right\}}{N},
$$

where $N$ is the number of $\mathrm{H}_{2}$ in the system. The results are shown in Table 2. $\triangle E$ is calculated on the condition that hydrogen is adsorbed on all adsorption sites as shown in Fig. 11. The radius of $\mathrm{B}$ ring, $r_{1}$, and the distance between $\mathrm{B}$ and $\mathrm{H}, r_{2}$, are investigated as geometric parameters with keeping their symmetry of nanowires (Fig. 11). In the case of the pentagonal AlB nanowire and $\mathrm{H}$ atoms, $r_{1}$ and $r_{2}$ are determined at $1.47 \AA$ and $1.20 \AA$, respectively. In this structure, the bond length between $\mathrm{B}$ atoms on the same ring is 1.73 $\AA$ and that between $\mathrm{B}$ and $\mathrm{Al}$ atoms is $2.50 \AA$. The stabilization energy $\triangle E$ is $-2.14 \mathrm{eV}$. In the hexagonal AlB nanowire, $r_{1}\left(r_{2}\right)$ are $1.68(1.20) \AA$, the length between $\mathrm{B}$ ( $\mathrm{B}$ and $\mathrm{Al}$ ) atoms is $1.68(2.34) \AA$, and the stabilization energy $\triangle E$ is $-2.10 \mathrm{eV}$. In both models, the bond lengths between $\mathrm{B}$ atoms on the same ring are longer than those of $\mathrm{AlB}$ nanowire without $\mathrm{H}$ atoms. The stabilization energy is below $-2.0 \mathrm{eV}$. On the other hand, in the case of $\mathrm{Al}$ nanowire, the bond length between $\mathrm{Al}$ atoms on the same ring is $2.76 \AA$, 
which is shorter than $\mathrm{Al}$ nanowire without $\mathrm{H}$ atoms. The stabilization energy is $-0.5 \mathrm{eV}$. Therefore, $\mathrm{AlB}$ nanowires are also more stable than $\mathrm{Al}$ nanowire for hydrogen adsorption. This difference of the hydrogen adsorption energy comes from the effect of the stabilization of the localized electron in HOMO. Since the adsorption energy of a $\mathrm{H}_{2}$ molecule adsorption is $-1.83 \mathrm{eV}$ in the $\mathrm{AlB}_{2}$ bulk surface model 1 and $-1.12 \mathrm{eV}$ in the surface model 2 in the case of $\mathrm{AlB}_{2}$, these results mean that $\mathrm{AlB}$ nanowires are more stable than $\mathrm{AlB}_{2}$ bulk surface for hydrogen adsorption.

\subsubsection{Electronic structure}

The DOS and band structure of $\mathrm{AlB}$ and $\mathrm{Al}$ simple nanowires after $\mathrm{H}$ adsorption are shown in Fig. 12. The DOS of $\mathrm{AlB}_{2}$ bulk surface models after hydrogen adsorption are shown in Fig. 13. The DOS and band structure of all models are metallic. In both AlB simple nanowires, the state on the Fermi level consists of narrow band. Thus the conductivity of AlB simple nanowire is different from the $\mathrm{AlB}_{2}$ bulk and surface.

The electron density on the $x z$-plane and $x y$-plane are shown in Fig. 14 . Figures 14(a),(b),(d),(e),(g), and (h) are the sum of the occupied states and (c),(f) and (i) are the highest occupied state. Figures 14(a),(d), and (g) show electron density on $x y$-plane and the other figures show that of $x z$-plane. In Fig. 15, the kinetic energy density and the tension density is shown. Figures $15(\mathrm{a}),(\mathrm{b}),(\mathrm{d}),(\mathrm{e}),(\mathrm{g})$, and $(\mathrm{h})$ are the sum of the occupied states and (c),(f) 
and (i) are the highest occupied state. The tension density is normalized. Figures 15(a),(d),(g) show the kinetic energy density and tension density on $x y$-plane and the other figures show those of $x z$-plane. Figure 16 shows the largest eigenvalue and eigenvector of stress tensor density. The eigenvector is normalized. Figures 16(a),(d), and (g) show electron density on $x y$-plane and the other figures show those of $x z$-plane.

First, we discuss the pentagonal AlB nanowire. After the hydrogen adsorption on the pentagonal AlB nanowire, the sum of the electron density in Figs. 14(a) and (b) appear between B and H. In the highest occupied state (Fig. 14(c)), the electron density also appears around $\mathrm{H}$ atoms. In the kinetic energy density (Figs. 15(a),(b) and (c) ), the sum of the all occupied states shows the same tendency in the electron density. The kinetic energy density regions with negative kinetic energy density for the highest occupied state appear at the center of the five-membered ring. This means that the highest occupied state consists of H states. Thus the peak around the Fermi level in the DOS figure is the electron related with $\mathrm{B}-\mathrm{H}$ bonds. In Figs. 16(a) and (b), the positive region and the spindle structure are seen between $\mathrm{B}$ and $\mathrm{H}$ and between $\mathrm{B}$ atoms on the same ring. This means that the both bonds are the covalent bond. The AlB hexagonal nanowire similar properties to the pentagonal model as seen in Figs. 12, 14, 15 and 16.

In $\mathrm{Al}$ nanowire, it is seen in Fig. 12 the DOS of $\mathrm{Al}$ nanowire on the Fermi level is changed by the hydrogen adsorption. The electron density of the sum of the all occupied state is high around $\mathrm{H}$ atoms. In the highest occupied state, the 
electron density and the kinetic energy density are localized between $\mathrm{Al}$ and $\mathrm{H}$ and between atoms on the axis. This is different from the electron density before the hydrogen adsorption. In Figs. 16(e) and (f), the positive region and the spindle structure are seen between $\mathrm{Al}$ and $\mathrm{H}$ and the negative region is seen between $\mathrm{Al}$ atoms. This means that the $\mathrm{Al}-\mathrm{H}$ bond is the covalent bond and $\mathrm{Al}-\mathrm{Al}$ bond is metallic.

\section{Conclusion}

We performed first-principles calculations for AlB nanowires to study the transitions of their electronic structures caused by $\mathrm{H}$ atoms adsorption. The difference between $\mathrm{AlB}$ nanowires and $\mathrm{Al}$ nanowire is clearly seen in the electron density and energy density. The electron of the highest occupied state appears on the direction perpendicular to the wire axis like dangling bonds. Therefore, it can be concluded that the AlB nanowires do not have the conductivity, while $\mathrm{AlB}_{2}$ bulk has it. It can be also seen that $\mathrm{AlB}$ nanowires are more stable in the hydrogen adsorption. After the hydrogen adsorption occurred, $\mathrm{H}$ atoms make the covalent bond with B and Al. There is no significant difference of the electron density between the hexagonal AlB nanowire and the pentagonal within this work. The DOS of the Al nanowire on the Fermi level is decreased by the $\mathrm{H}$ adsorption. We have confirmed that conductivity of AlB nanowires are unchanged with hydrogen adsorption as seen from the tension density. 
The hydrogen adsorption energy of AlB nanowire models is larger than that of $\mathrm{Al}$ nanowire. In this point, $\mathrm{AlB}$ nanowire is more stable in the hydrogen adsorption.

\section{Acknowledgements}

A part of the computations was supported by Center for Computational Science, Okazaki, Japan. This work is supported in part by the Center of Excellence for Research and Education on Complex Functional Mechanical Systems (COE Program of the Ministry of Education, Culture, Sports, Science and Technology, Japan). 


\section{References}

[1] H. Nakano, H. Ohta, A. Yokoe, K. Doi, and A. Tachibana, J. Pow. Sour. $163(2006) 125$.

[2] A. Goldberg and I. Yarovsky, Phys. Rev. B 75 (2007) 195403.

[3] H. Cheng, A. C. Cooper, and G. P. Pez, J. Am. Chem. Soc. 123 (2001) 5845; L. Chen, A. C. Cooper, G. P. Pez, and H. Cheng, J. Phys. Chem. C, in press.

[4]T. Makita, K. Doi, K. Nakamura, and A. Tachibana, J. Chem. Phys. 119 (2003) 538.

[5]Y. Kawakami, Y. Nojima, K. Doi, K. Nakamura, and A. Tachibana, Electrochim. Acta 50 (2004) 739.

[6]M. Suzuki, K. Nagai, S. Kinoshita, K. Nakajima, K. Kimura, T. Okano, and K. Sasakawa, Appl. Phys. Lett. 89 (2006) 133103.

[7]A. Tachibana, J. Chem. Phys. 115 (2001) 3497.

[8]A. Tachibana, in Stress Induced Phenomena in Metallization, edited by S.

P. Baker (American Institute of Physics, New York, 2002) pp.105-116.

[9]A. Tachibana, in Fundamental Perspectives in Quantum Chemistry: A Tribute to the Memory of Per-Olov Löwdin, edited by E. Brändas, and E. Kryachko (Kluwer Academic, Dordrecht, 2003), Vol. II, pp.211-239.

[10]A. Tachibana, Int. J. Quantum Chem. Symp. 21 (1987) 181.

[11]A. Tachibana and R. G. Parr, Int. J. Quantum Chem. 41 (1992) 527.

[12]A. Tachibana, Int. J. Quantum Chem. 57 (1996) 423. 
[13]A. Tachibana, Theor. Chem. Acc. 102 (1999) 188.

[14]I. Loa, K. Kunc, and K. Syassen, Phys. Rev. B 66 (2002) 134101.

[15]Periodic Regional DFT program package, ver.2, (Tachibana Lab., Kyoto Univ., Kyoto, 2002).

[16]D. R. Hamann, Phys. Rev. B 40 (1989) 2980.

[17]J. P. Perdew and Y. Wang, Phys. Rev. B 45 (1992) 13244.

[18]M. J. Frisch et al., Gaussian 03, Revision B.05, Gaussian, Inc., Pittsburgh PA 2003.

[19]G. te Velde, F. M. Bickelhaupt, S. J. A. van Gisbergen, C. Fonseca Guerra, E. J. Baerends, J. G. Snijders, and T. Ziegler, J. Comput. Chem. 22 (2001) 931. 
Table 1

The geometry parameters of nanowiremodels and $\mathrm{AlB}_{2}$ bulk

\begin{tabular}{lccc}
\hline & Site 1 & Site 2 & Site 3 \\
\hline AlB Pentagonal & $-5.53[\mathrm{eV}]$ & $-3.54[\mathrm{eV}]$ & - \\
AlB Hexagonal & $-5.65[\mathrm{eV}]$ & $-4.53[\mathrm{eV}]$ & - \\
Al pentagonal & $-3.59[\mathrm{eV}]$ & $-3.34[\mathrm{eV}]$ & $-3.37[\mathrm{eV}]$ \\
\hline
\end{tabular}

Table 2

Geometry parameters and the stabilization energy of $\mathrm{H}_{2}$ molecule adsorption

\begin{tabular}{lccc} 
& $r_{1}(\AA)$ & $r_{2}(\AA)$ & $\Delta \mathrm{E}(\mathrm{eV})$ \\
\hline AlB Pentagonal & 1.47 & 1.20 & -2.14 \\
AlB Hexagonal & 1.68 & 1.20 & -2.10 \\
Al Pentagonal & 2.35 & 1.57 & -0.50 \\
AlB Surface 1 & - & - & -1.84 \\
AlB Surface 2 & - & - & -1.12
\end{tabular}




\section{Figure captions}

Figure 1. (a) $\mathrm{AlB}_{2}$ bulk model, (b) AlB larger hexagonal nanowire model, (c) AlB hexagonal nanowire model, (d) $\mathrm{AlB}_{2}$ bulk surface model 1, (e) $\mathrm{AlB}_{2}$ bulk surface model 2.

Figure 2. (a) Al pentagonal nanowire model, (b) AlB larger hexagonal nanowire model.

Figure 3. Total energy curves. (a) AlB pentagonal nanowire model as a function of $r_{1}$, (b) AlB pentagonal nanowire model as a function of $c$. (c) AlB hexagonal nanowire model as a function of $r_{1}$. (d) AlB hexagonal nanowire model as a function of $c$.

Figure 4. Total $\triangle E / \triangle c$ as a function of $\triangle c$. (a) AlB pentagonal nanowire model, (b) AlB hexagonal nanowire model.

Figure 5. DOS and band structure of AlB nanowire model. (a) AlB pentagonal nanowire model, (b) AlB hexagonal nanowire model, (c) Al pentagonal nanowire model. 
Figure 6. DOS and band structure of AlB lager hexagonal nanowire model and DOS of $\mathrm{AlB}_{2}$ bulk and bulk surface. (a) AlB larger hexagonal nanowire model, (b) $\mathrm{AlB}_{2}$ surface model 1, (c) $\mathrm{AlB}_{2}$ surface model 2, (d) $\mathrm{AlB}_{2}$ bulk model.

Figure 7. Electron density of nanowire models. The region in the circle is pseudopotential. Electron density of the sum of all occupied states in (a) and (b). AlB pentagonal, (d) and (e) AlB hexagonal, and (g) and (h) Al pentagonal nanowire models. Electron density of the highest occupied states (c) AlB pentagonal, (f) AlB hexagonal and (i) Al pentagonal nanowire models.

Figure 8. Kinetic energy density and tension density of nanowire models. The region in the circle is pseudopotential. The tension density is showed only on the region where the kinetic energy is positive. Kinetic energy density and tension density of the sum of all occupied states in (a) and (b) AlB pentagonal, (d) and (e) AlB hexagonal, and (g) and (h) Al pentagonal nanowire models. Light (dark) gray region means positive (negative) kinetic energy density. Kinetic energy density and tension density of the highest occupied states (c) AlB pentagonal, (f) AlB hexagonal, and (i) Al pentagonal nanowire models.

Figure 9. The largest eigenvalues of stress-tensor density. Red (blue) region 
means positive (negative) stress density. The eigenvectors are normalized. The largest eigenvalues and eigenvectors of stress-tensor density in (a) and (b) AlB pentagonal, (c) and (d) AlB hexagonal, and (e) and (f) Al pentagonal nanowire models.

Figure 10. $\mathrm{H}$ atom adsorption site. (Site 1) The top of $\mathrm{B}(\mathrm{Al})$ ring. (Site 2) The same distance from $\mathrm{B}(\mathrm{Al})$ atoms of the same ring. (Site 3) The same distance from $\mathrm{B}(\mathrm{Al})$ atoms of the different rings.

Figure 11. $\mathrm{H}$ absorbed simple nanowire models and $\mathrm{AlB}_{2}$ surfaces. (a) $\mathrm{AlB}$ pentagonal nanowire model. (b) AlB hexagonal nanowire model. (c) Al pentagonal nanowire model. (d) $\mathrm{AlB}_{2}$ surface model 1. (e) $\mathrm{AlB}_{2}$ surface model 2.

Figure 12. Total DOS and band structure of nanowire models after H adsorption. (a) AlB pentagonal nanowire model. (b) AlB hexagonal nanowire model. (c) Al pentagonal nanowire model.

Figure 13. Total DOS of surface models after $\mathrm{H}$ adsorption. (d) $\mathrm{AlB}_{2}$ surface model 1. (e) $\mathrm{AlB}_{2}$ surface model 2. 
Figure 14. Electron density of nanowire models after $\mathrm{H}$ adsorption. The region in the circle is pseudopotential. Electron density of the sum of all occupied states in (a) and (b) AlB pentagonal, (d) and (e) AlB hexagonal, and (g) and (h) Al pentagonal nanowire models. Electron density of the highest occupied states (c) AlB pentagonal, (f) AlB hexagonal and (i) Al pentagonal nanowire models.

Figure 15. Kinetic energy density and tension density of nanowire models after $\mathrm{H}$ adsorption. The region in the circle is pseudopotential. The tension density is showed only on the region where the kinetic energy is positive. Kinetic energy density and tension density of the sum of all occupied states in (a) and (b) AlB pentagonal, (d) and (e) AlB hexagonal, and (g) and (h) Al pentagonal nanowire models. Light (dark) gray region means positive (negative) kinetic energy density. Kinetic energy density and tension density of the highest occupied states (c) AlB pentagonal, (f) AlB hexagonal, and (i) Al pentagonal nanowire models.

Figure 16. The largest eigenvalues of stress-tensor density. Red (blue) region means positive (negative) stress density. The eigenvectors are normalized. The largest eigenvalues and eigenvectors of stress-tensor density in (a) and (b) AlB pentagonal, (c) and (d) AlB hexagonal, and (e) and (f) Al pentagonal nanowire models. 
KURENAI 仜

- $-909-900000000$ - 0 - 0 - 0 - 0 - 0 -

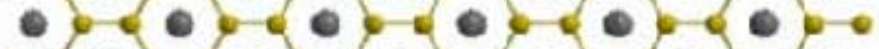

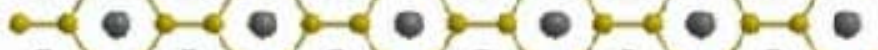

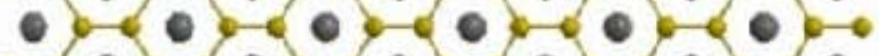

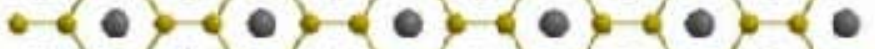

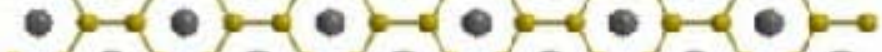
00 - 0 - 0 - 0 - 0 - 0

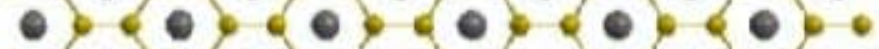
$\because 000-000000$

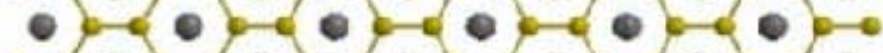
- 0 - 0 - 0 - 0 - 00 - 0

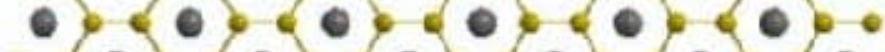

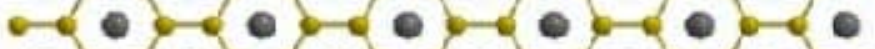

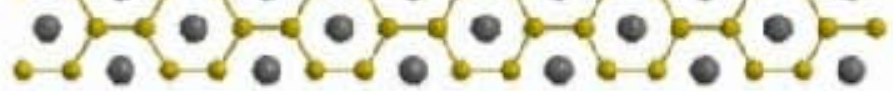

- $-10 \%$

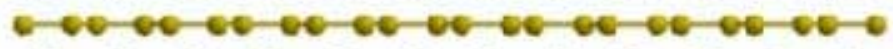
- - - -101

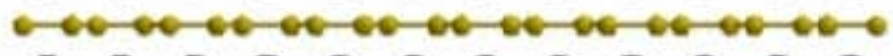

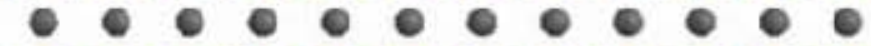

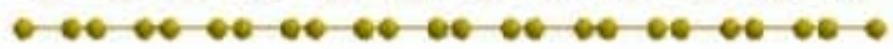

a
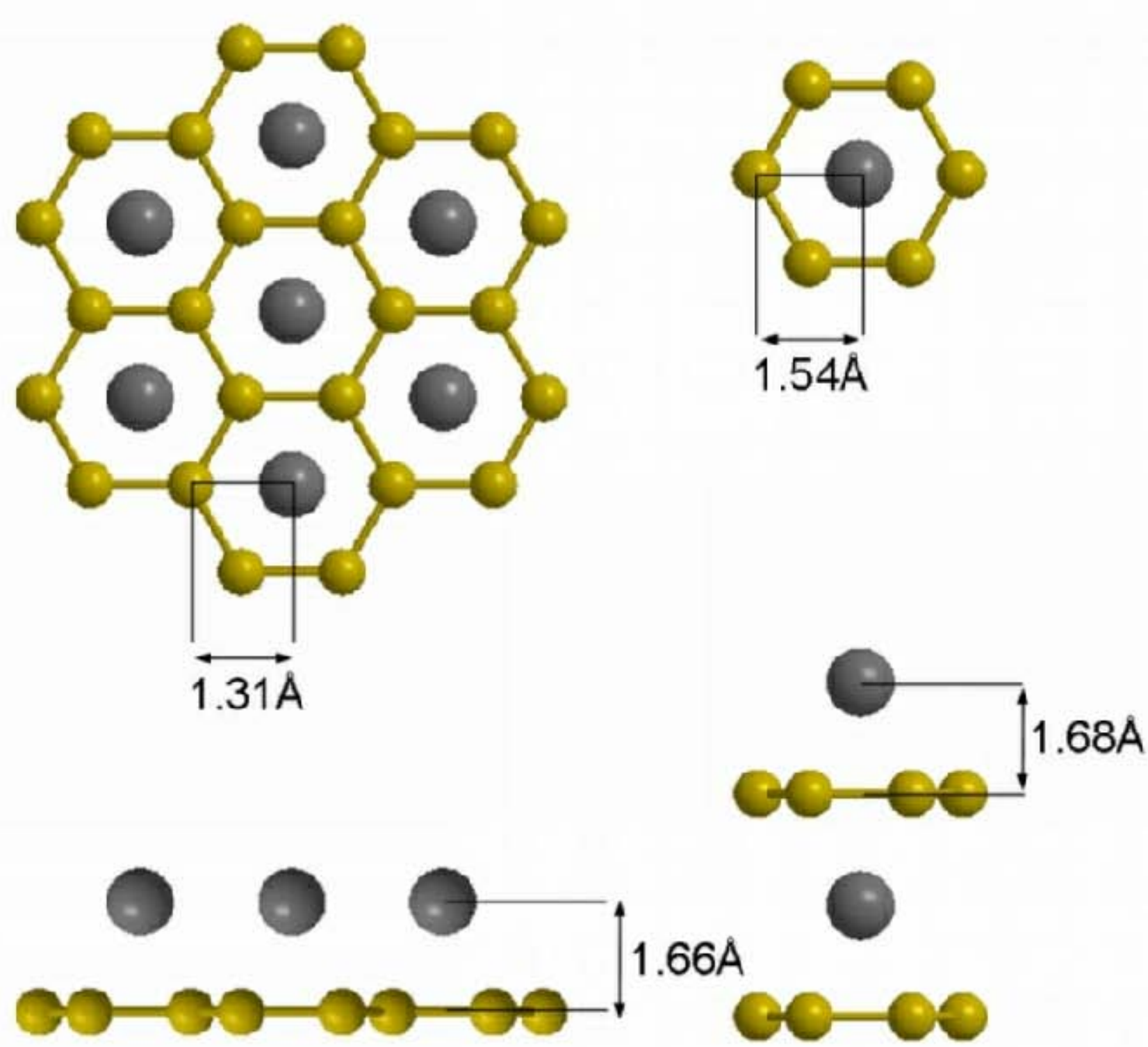

b

C 

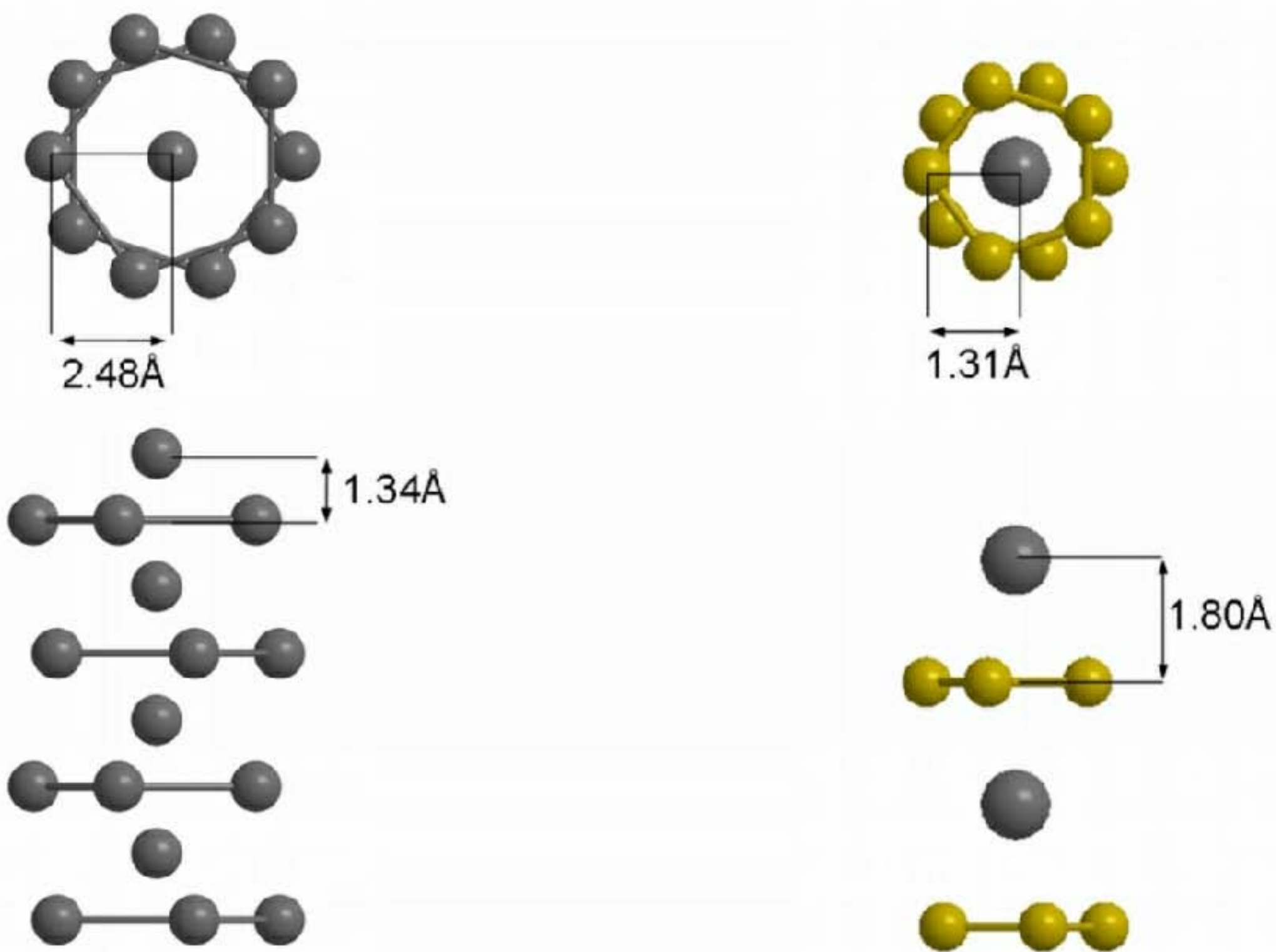

a

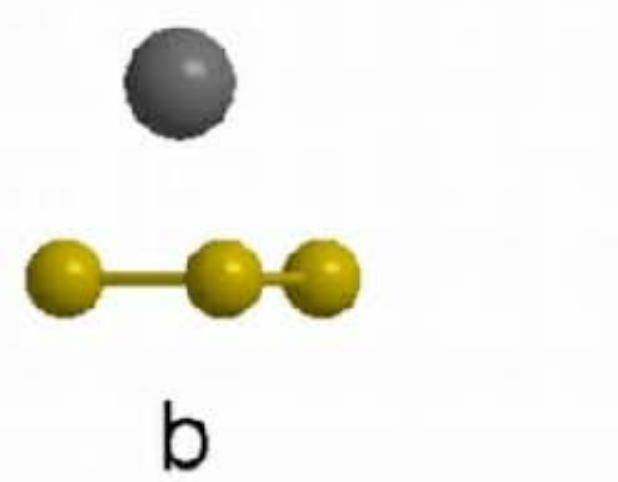




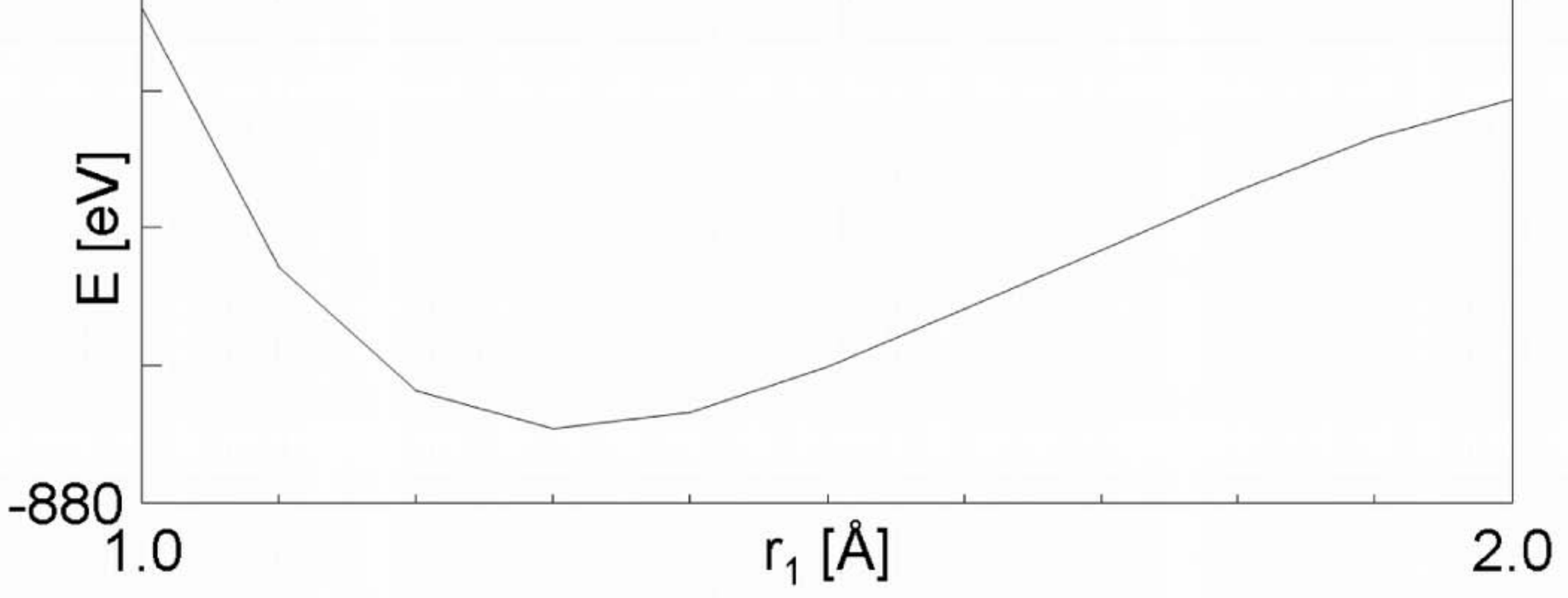




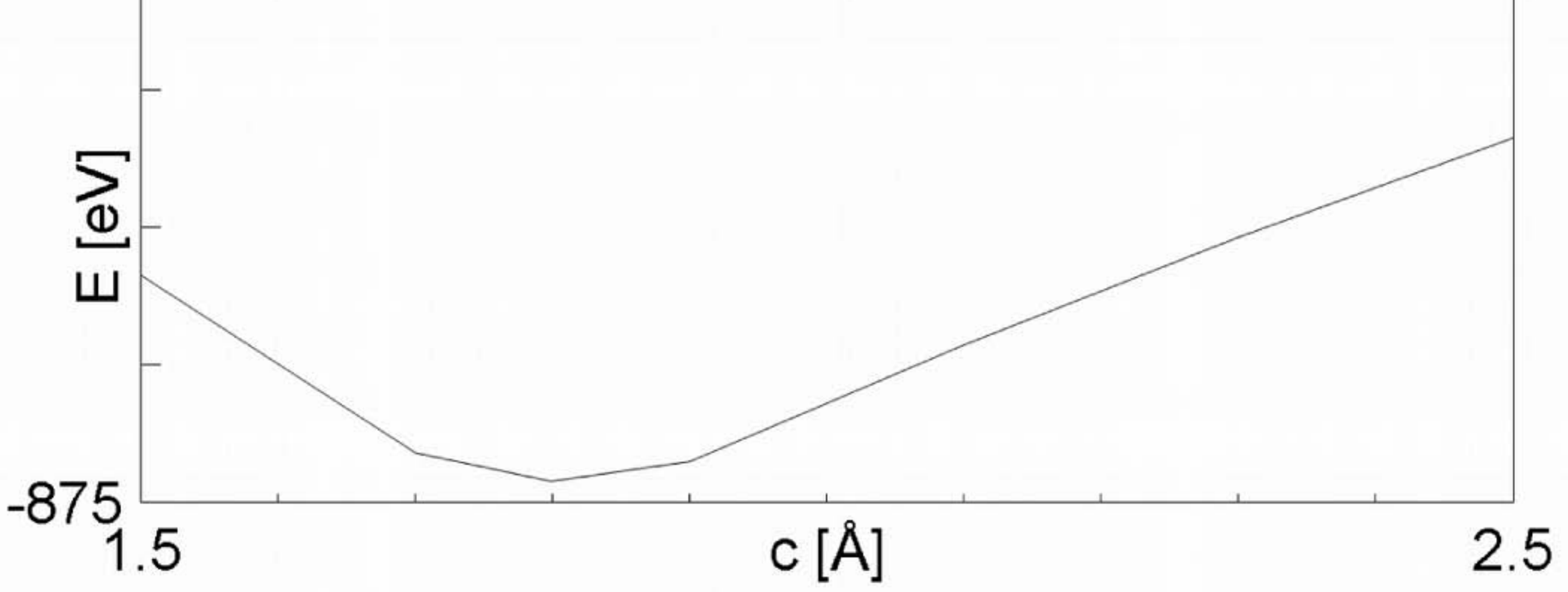




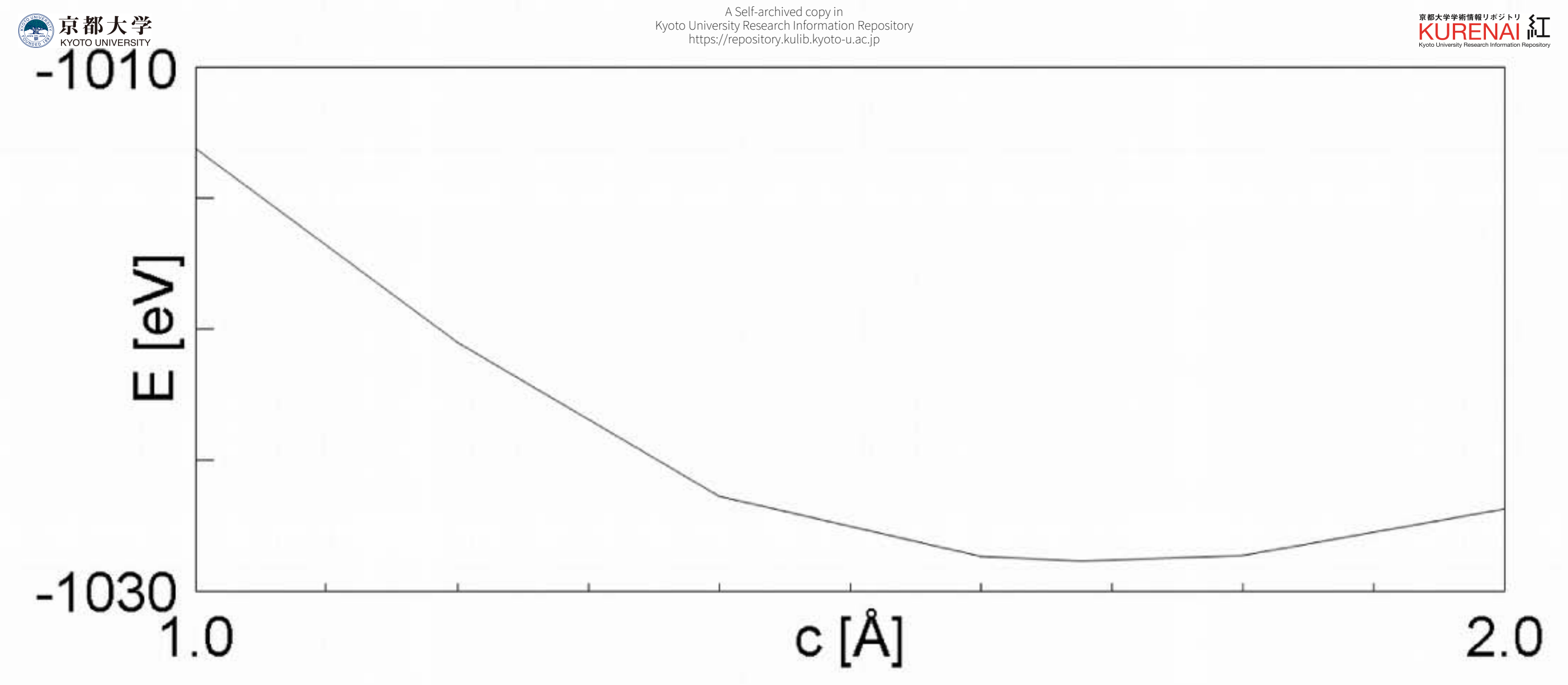




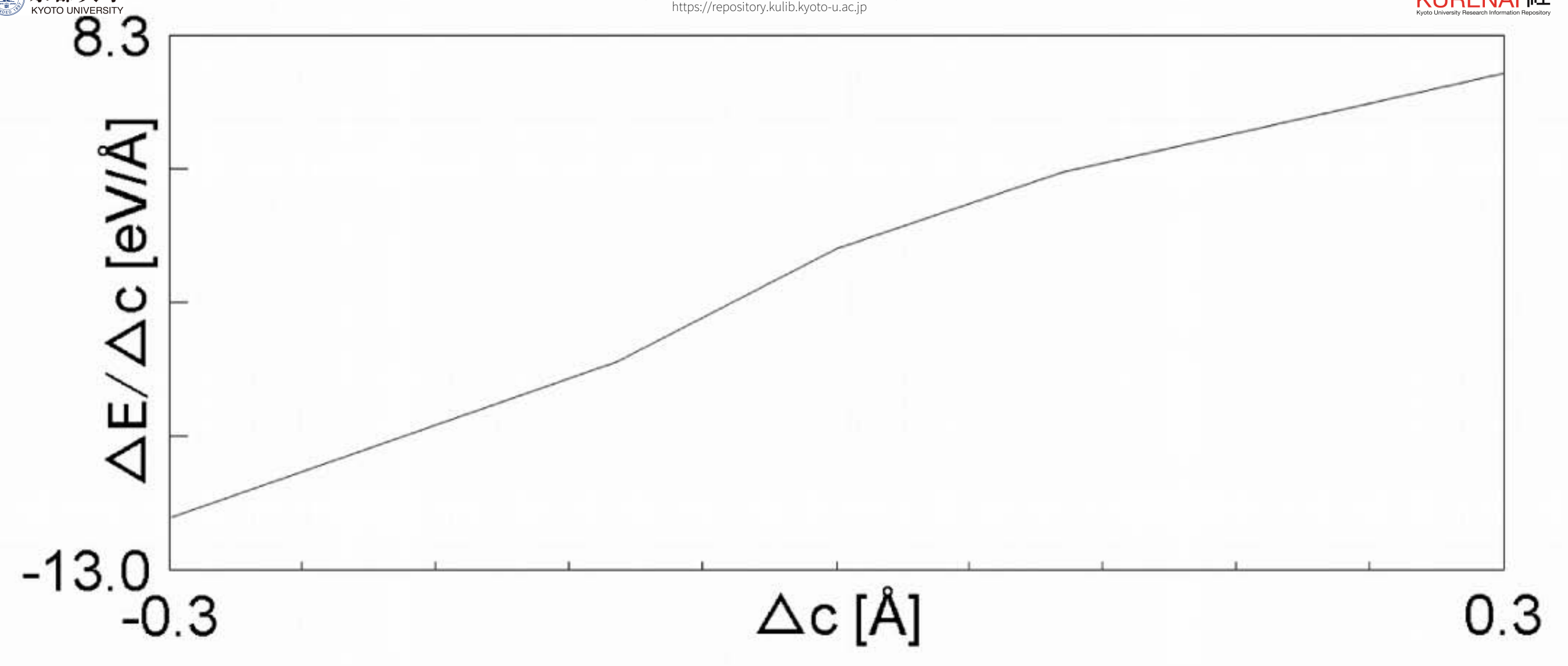


6.30

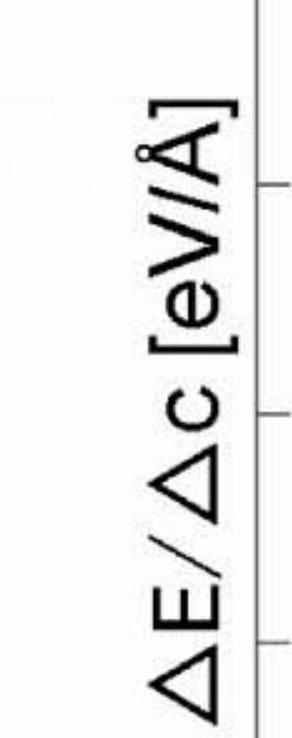

$-9.01$ $-0.3$ 


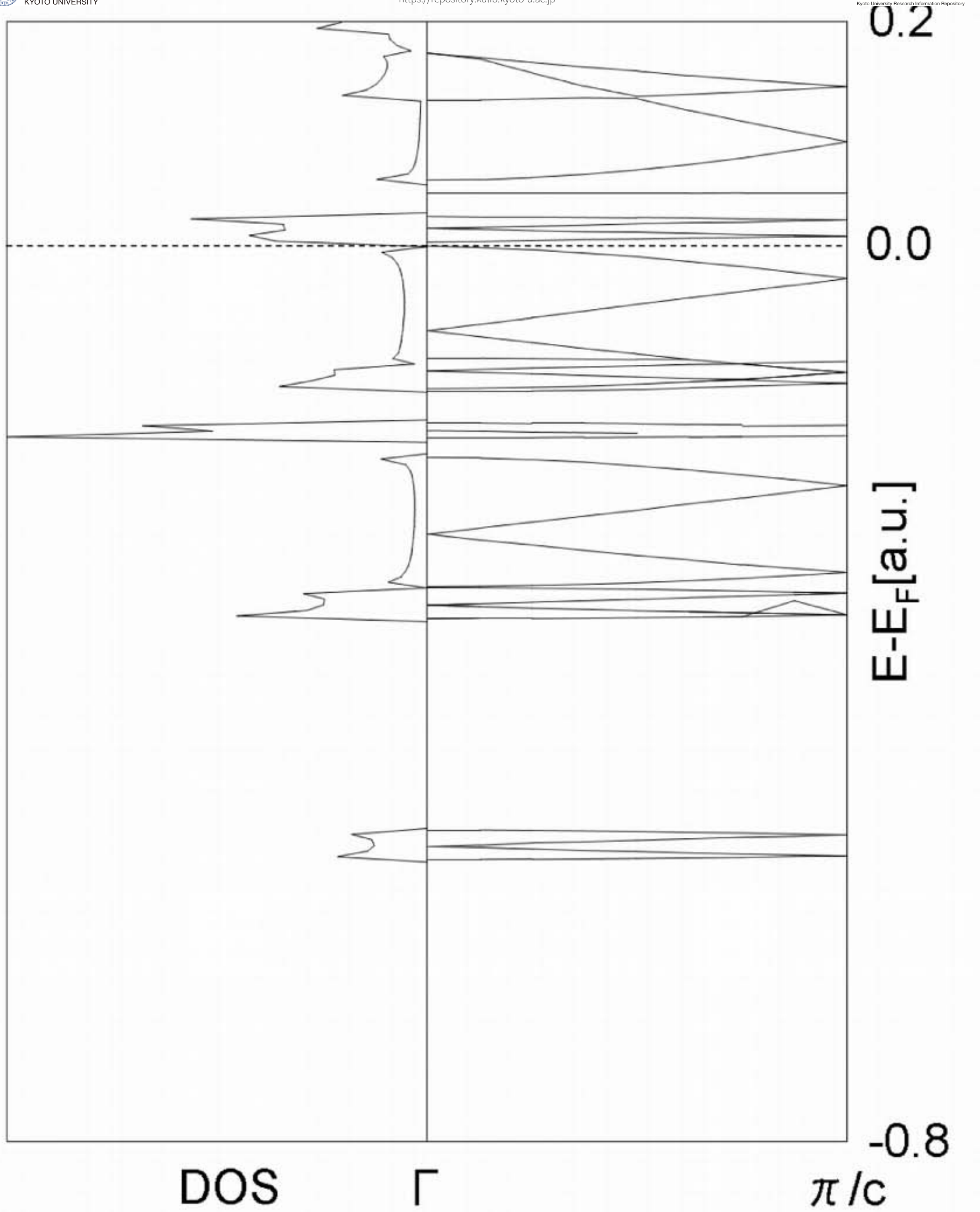




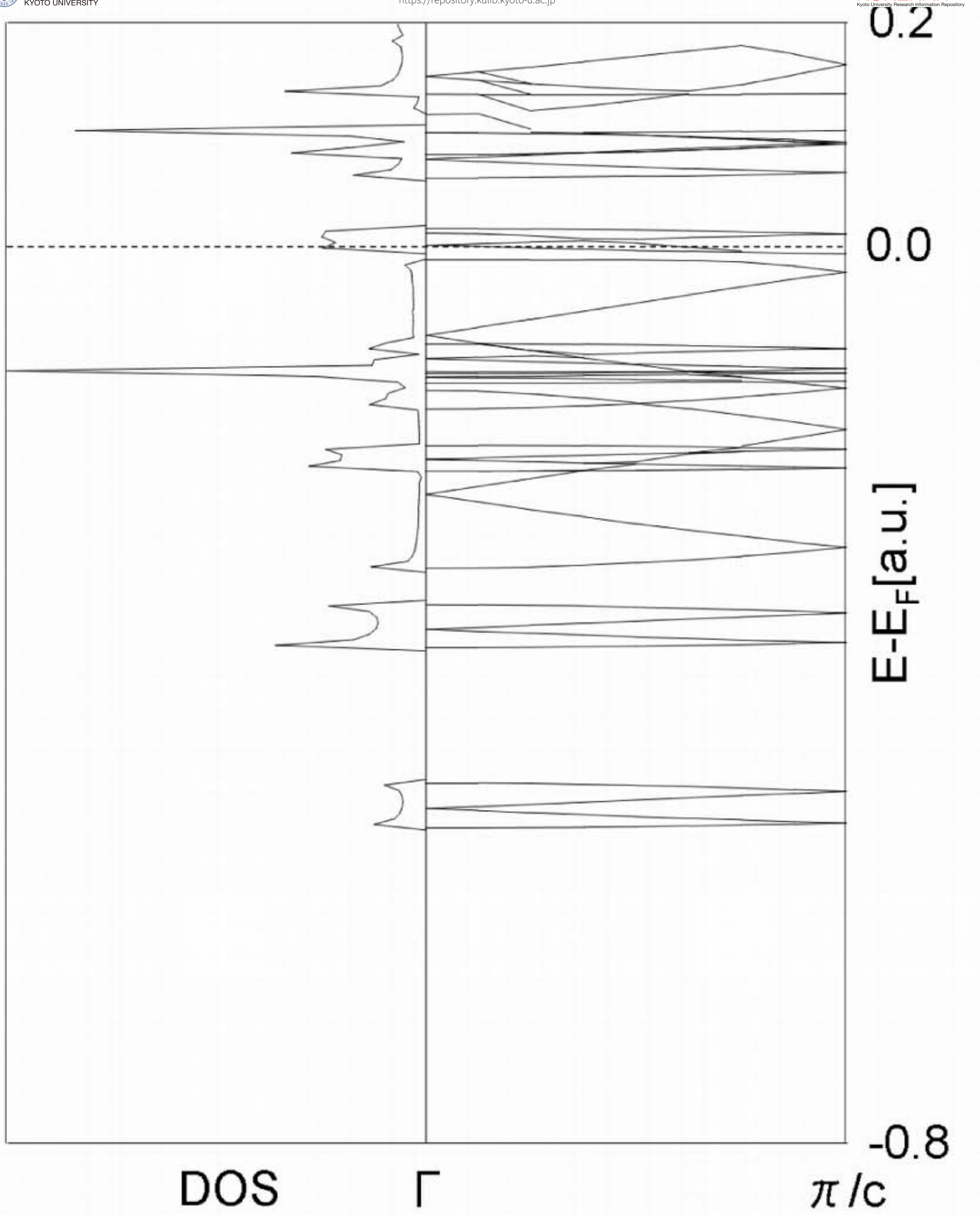




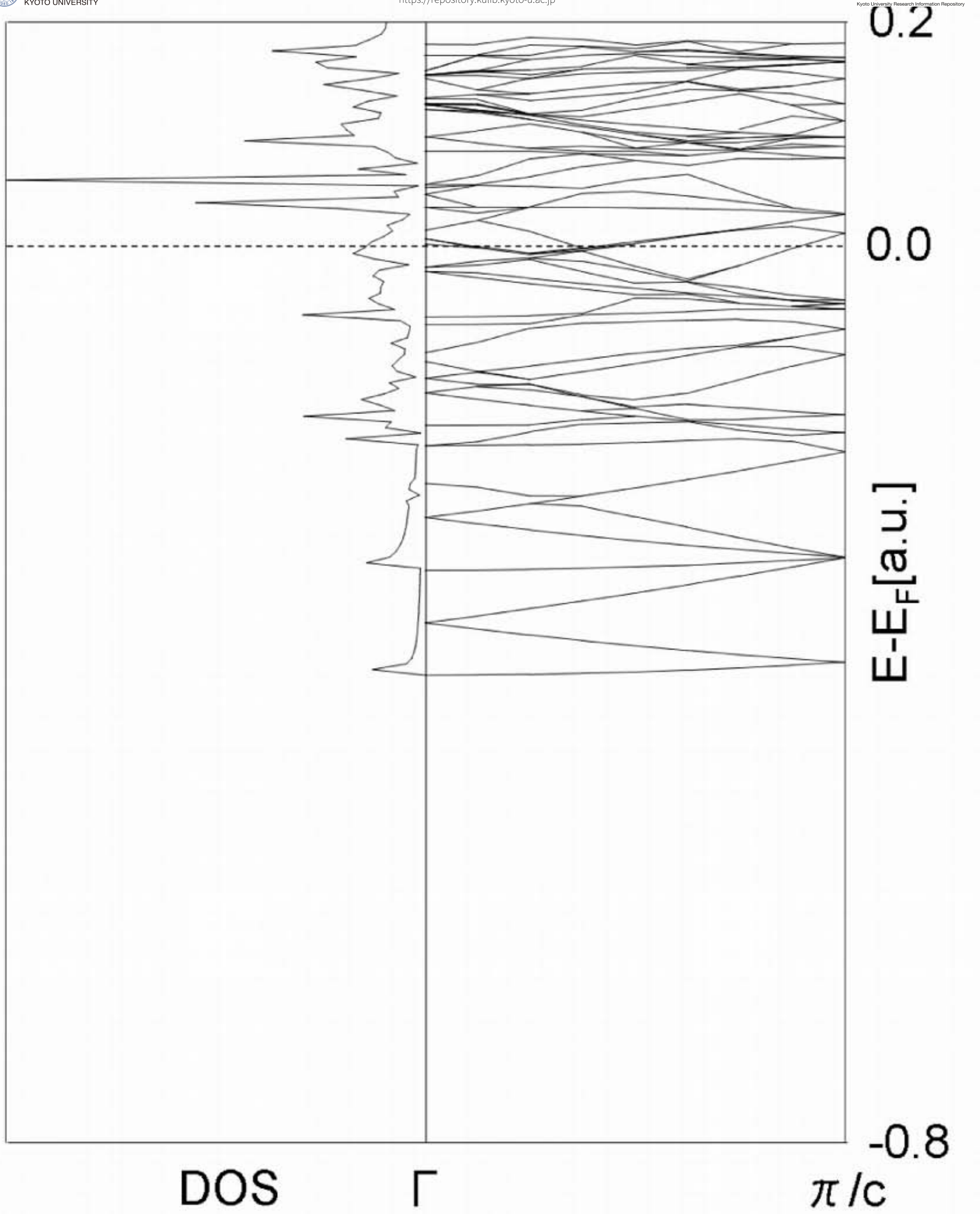




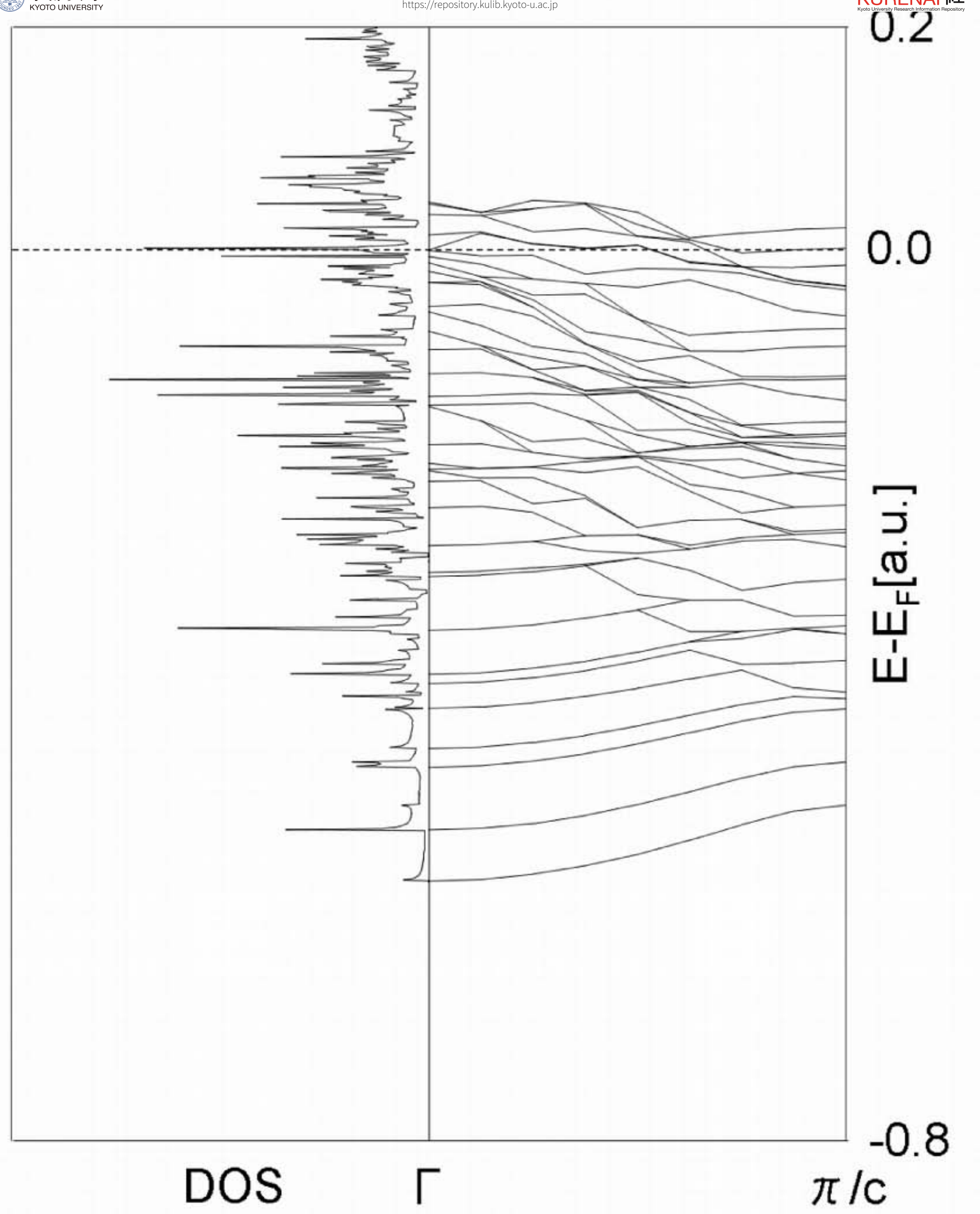

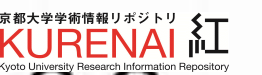




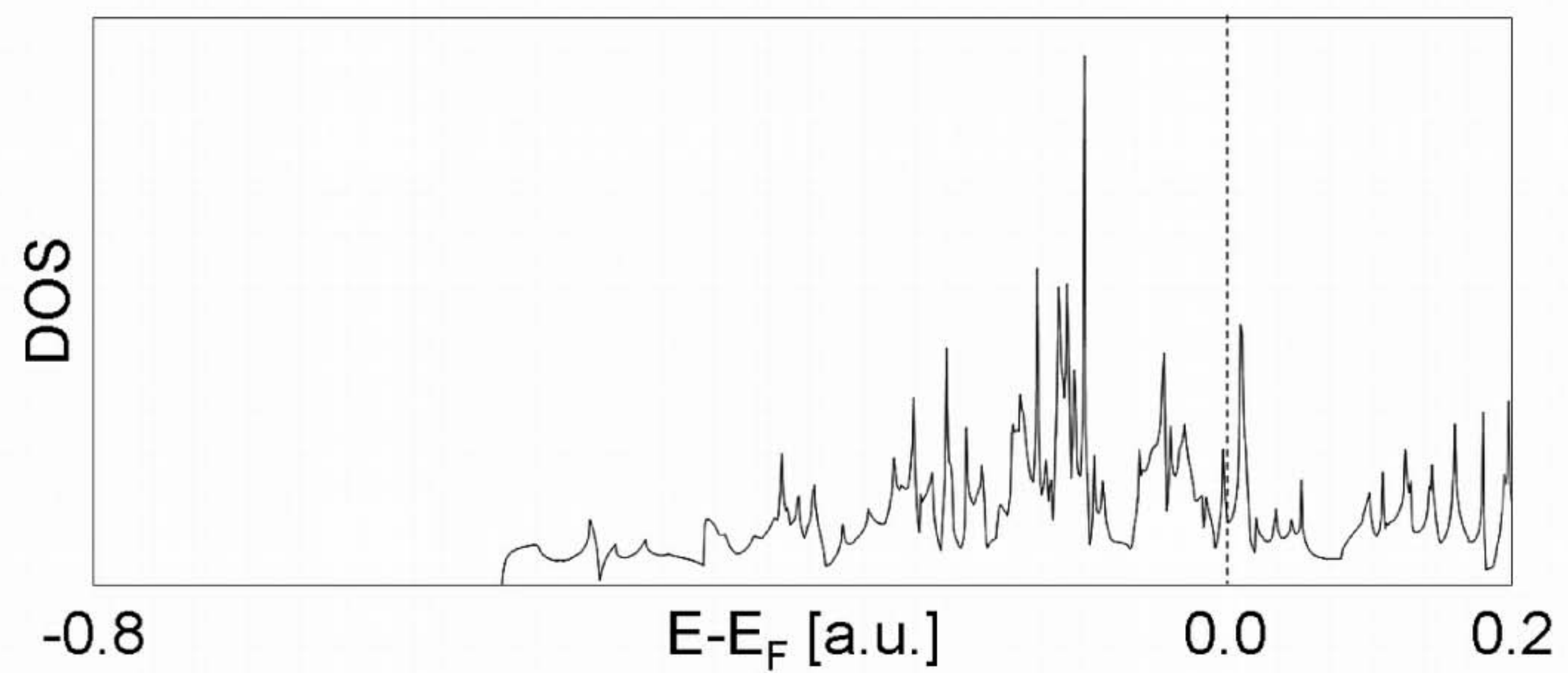




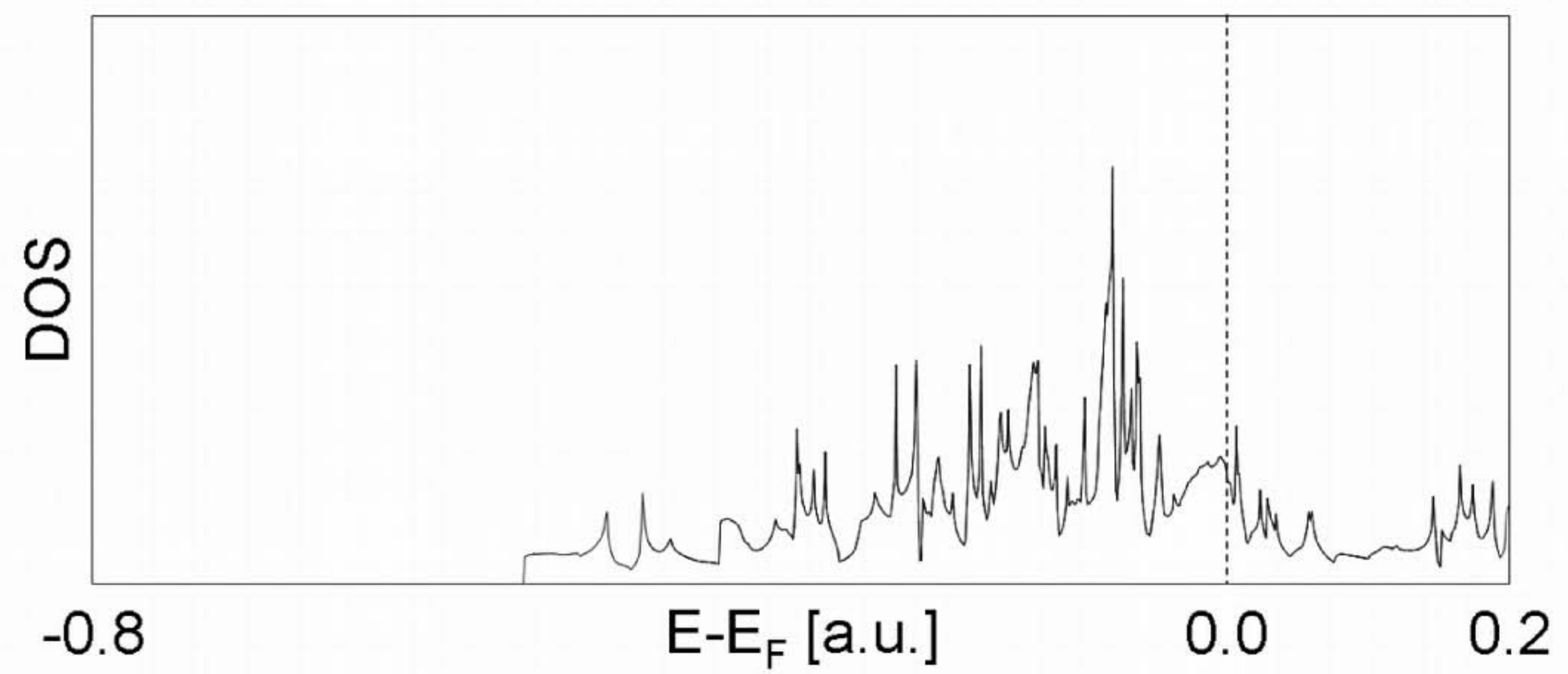




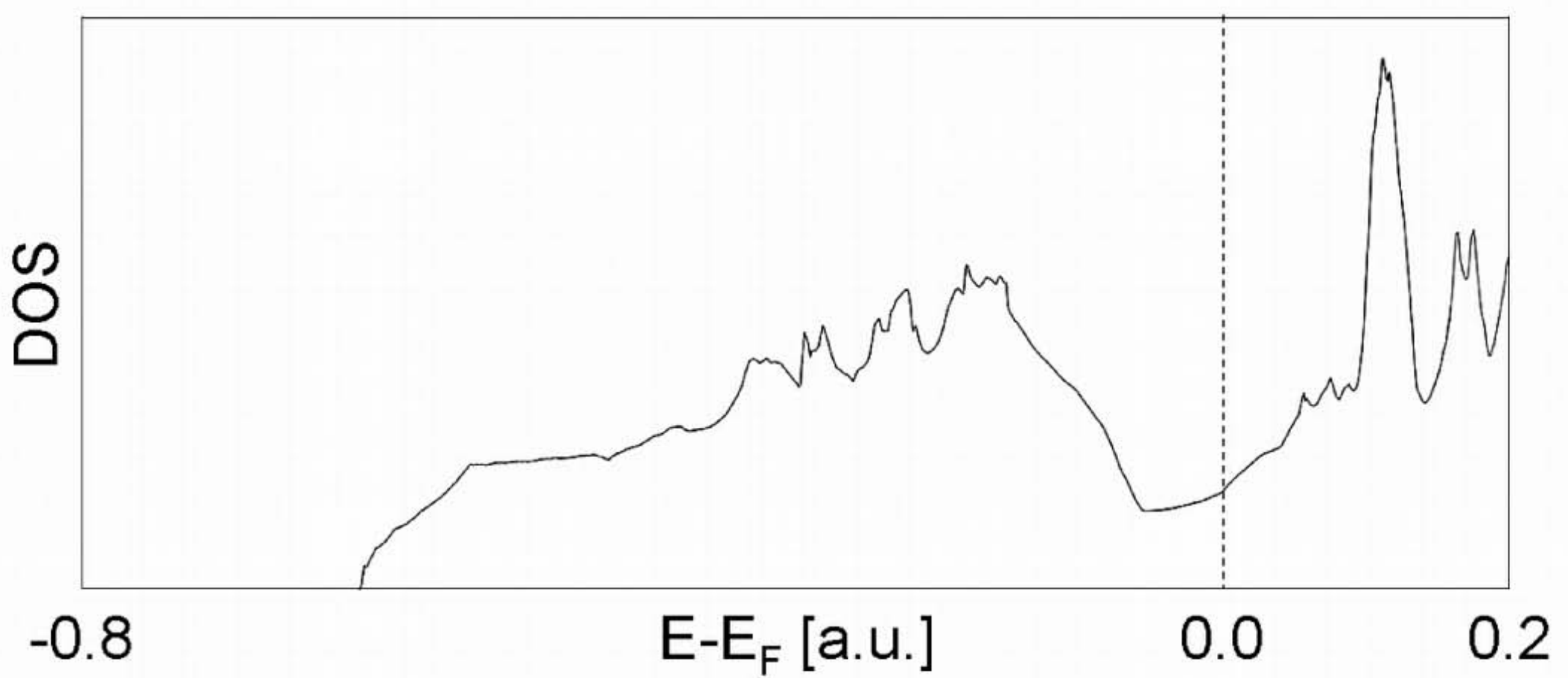




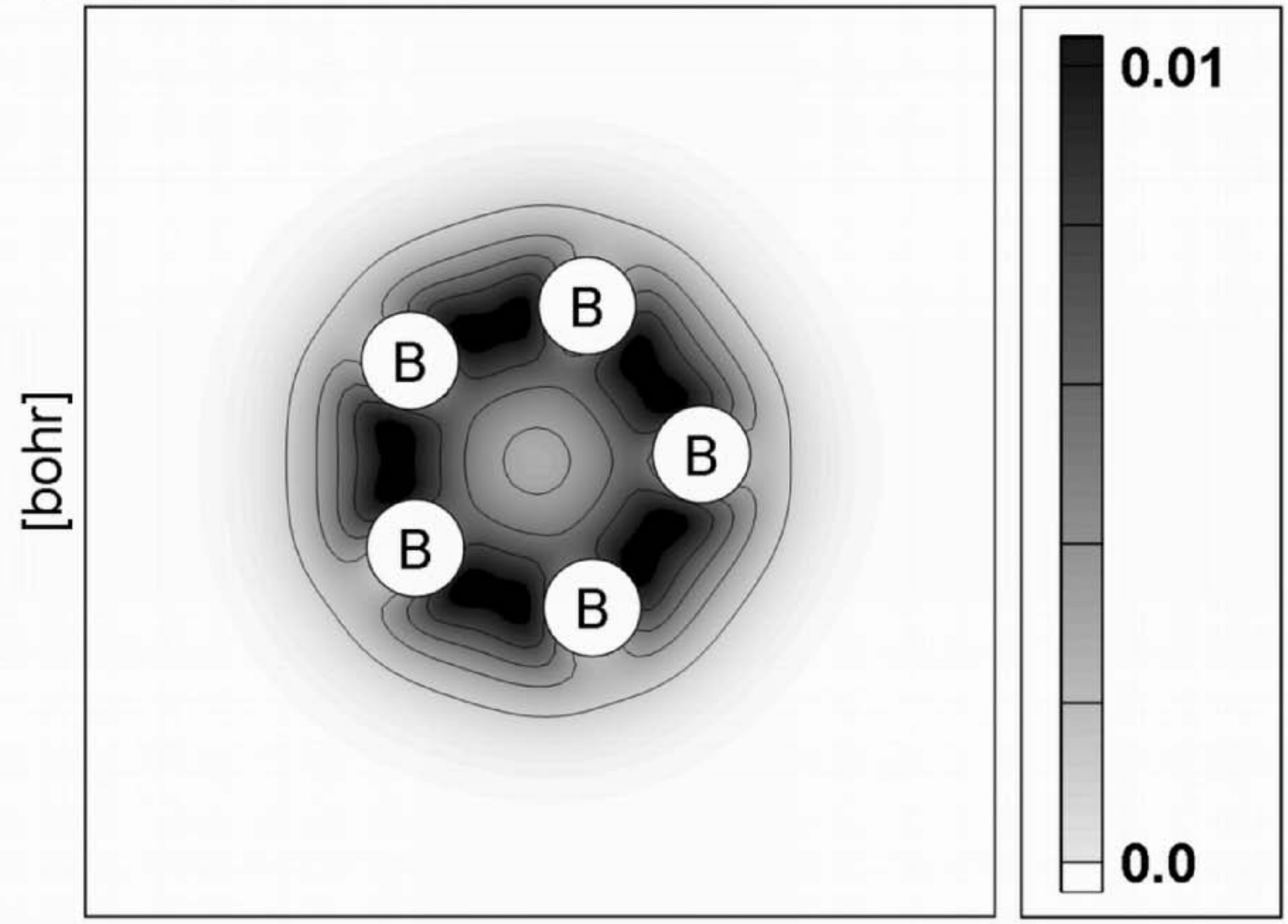




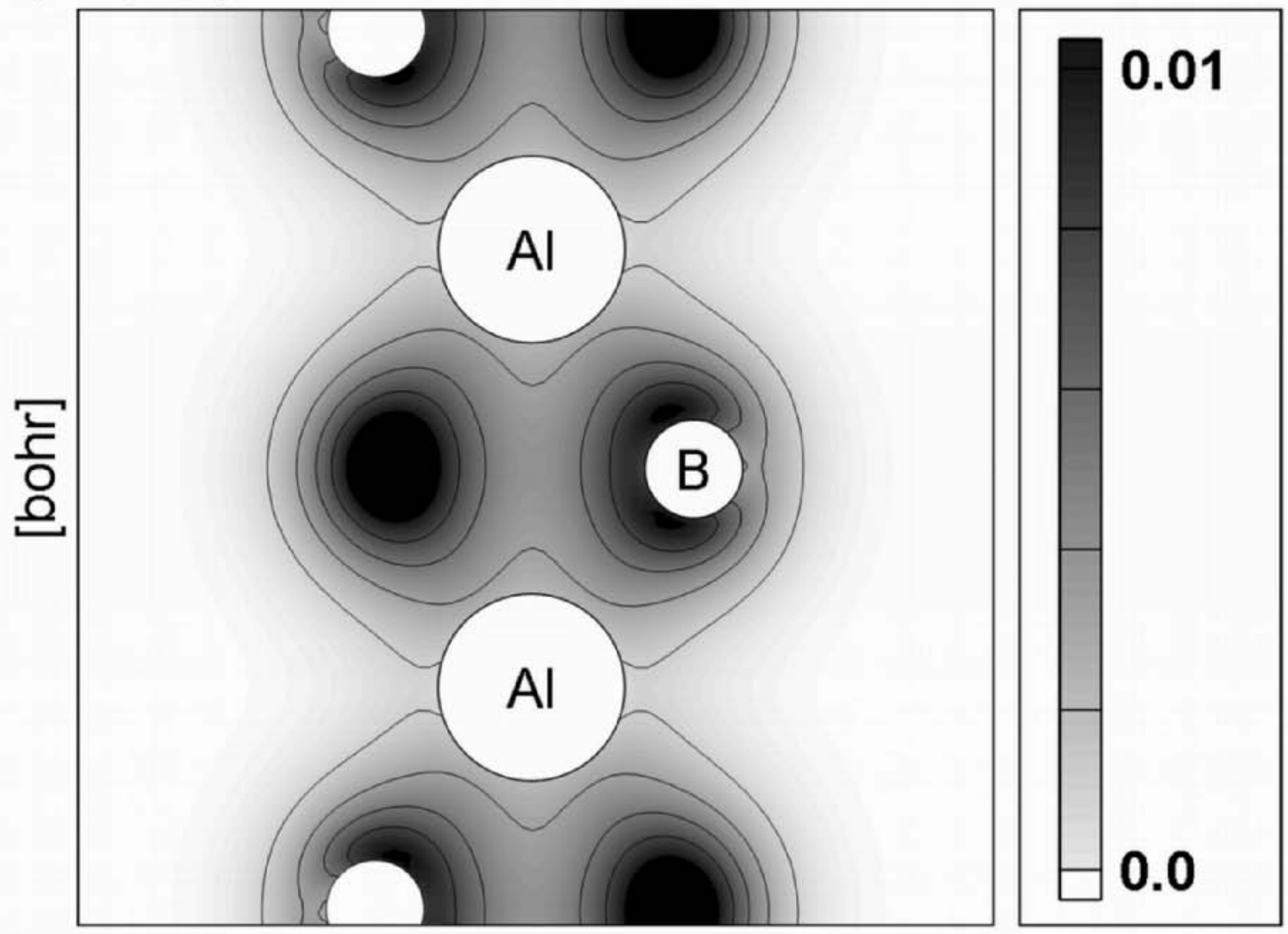




\section{$(-7.00,0.00,7.00)$}

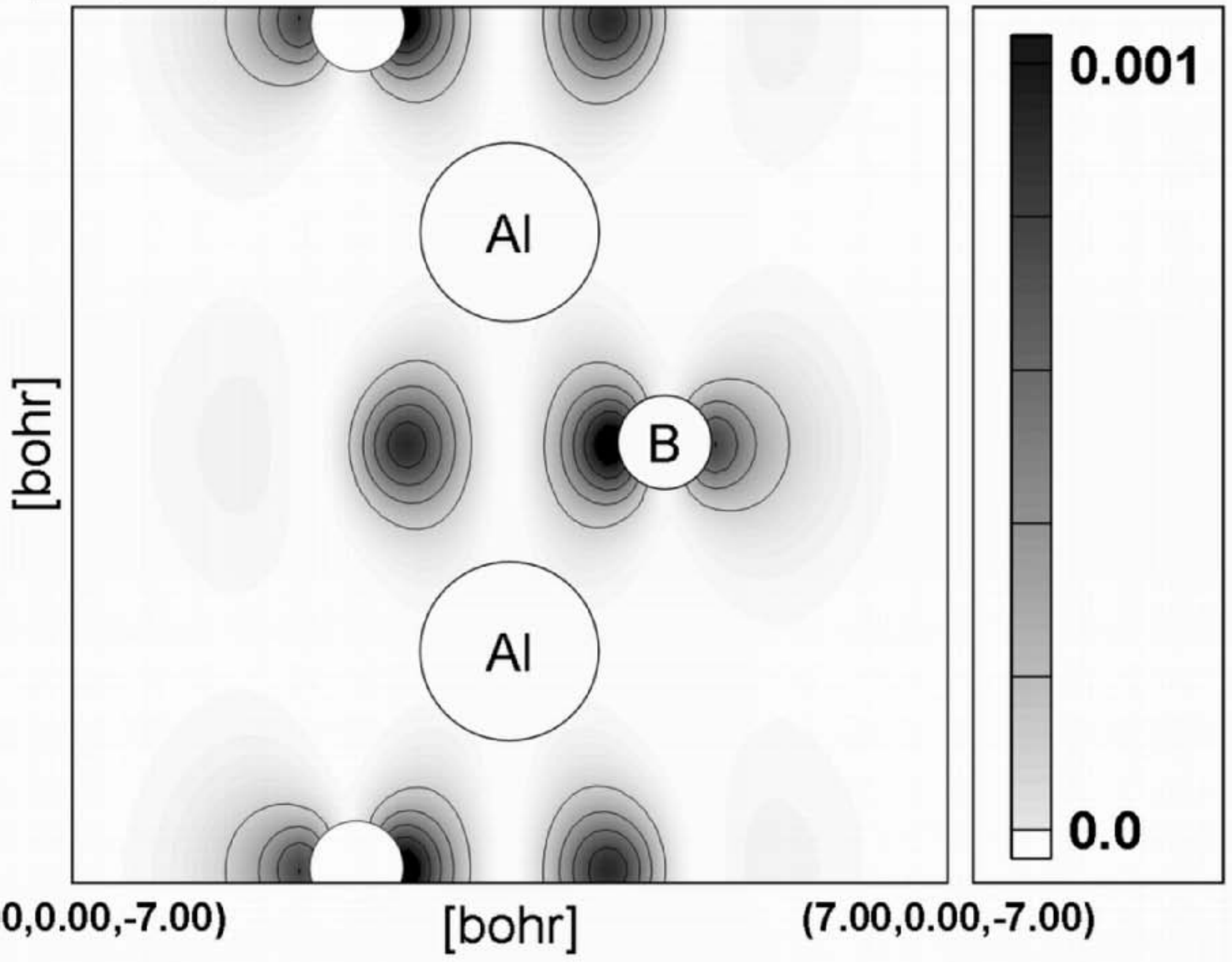

$(-7.00,0.00,-7.00)$

[bohr]

$(7.00,0.00,-7.00)$ 


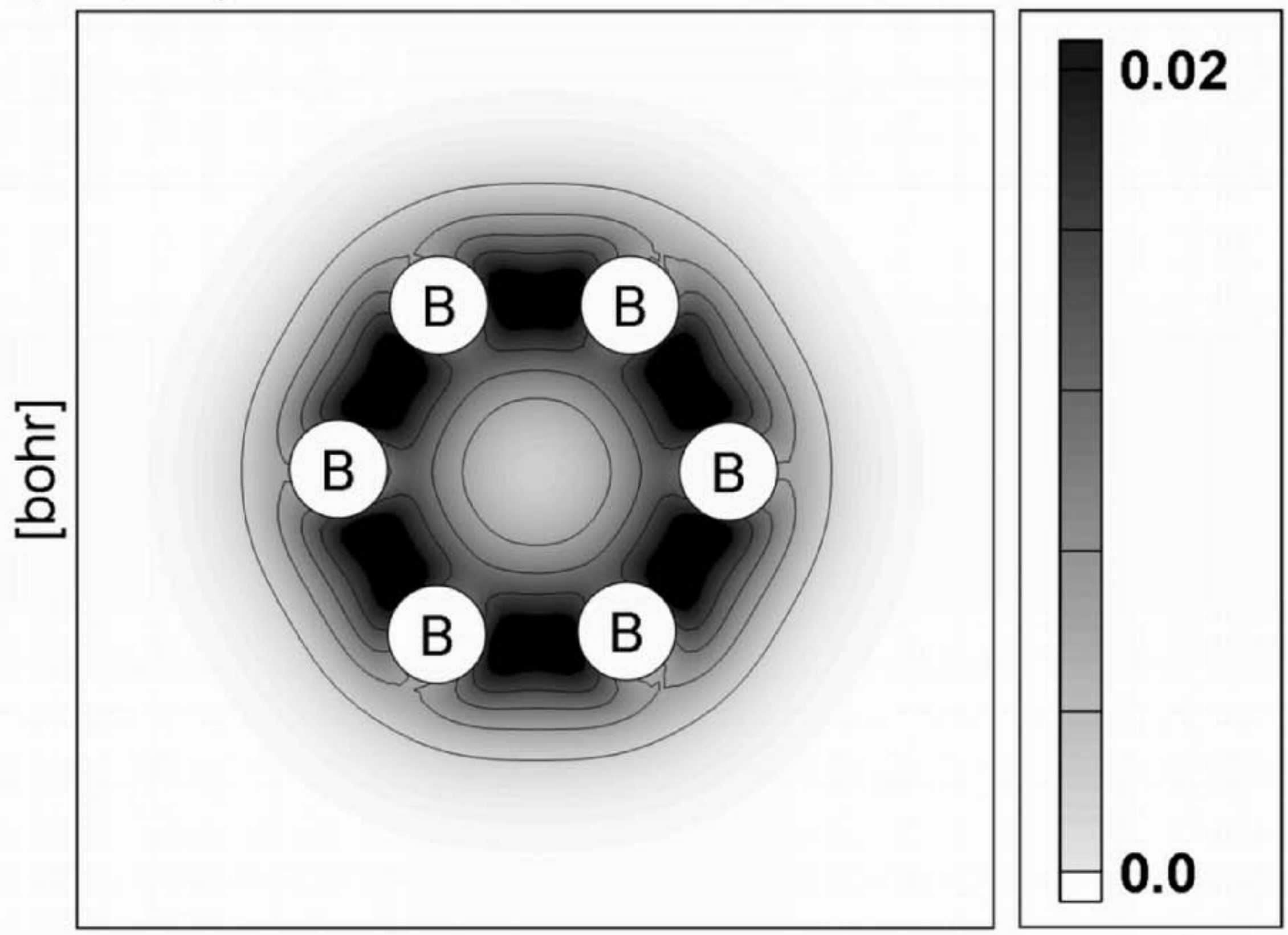




\section{$(-7.00,0.00,7.00)$}

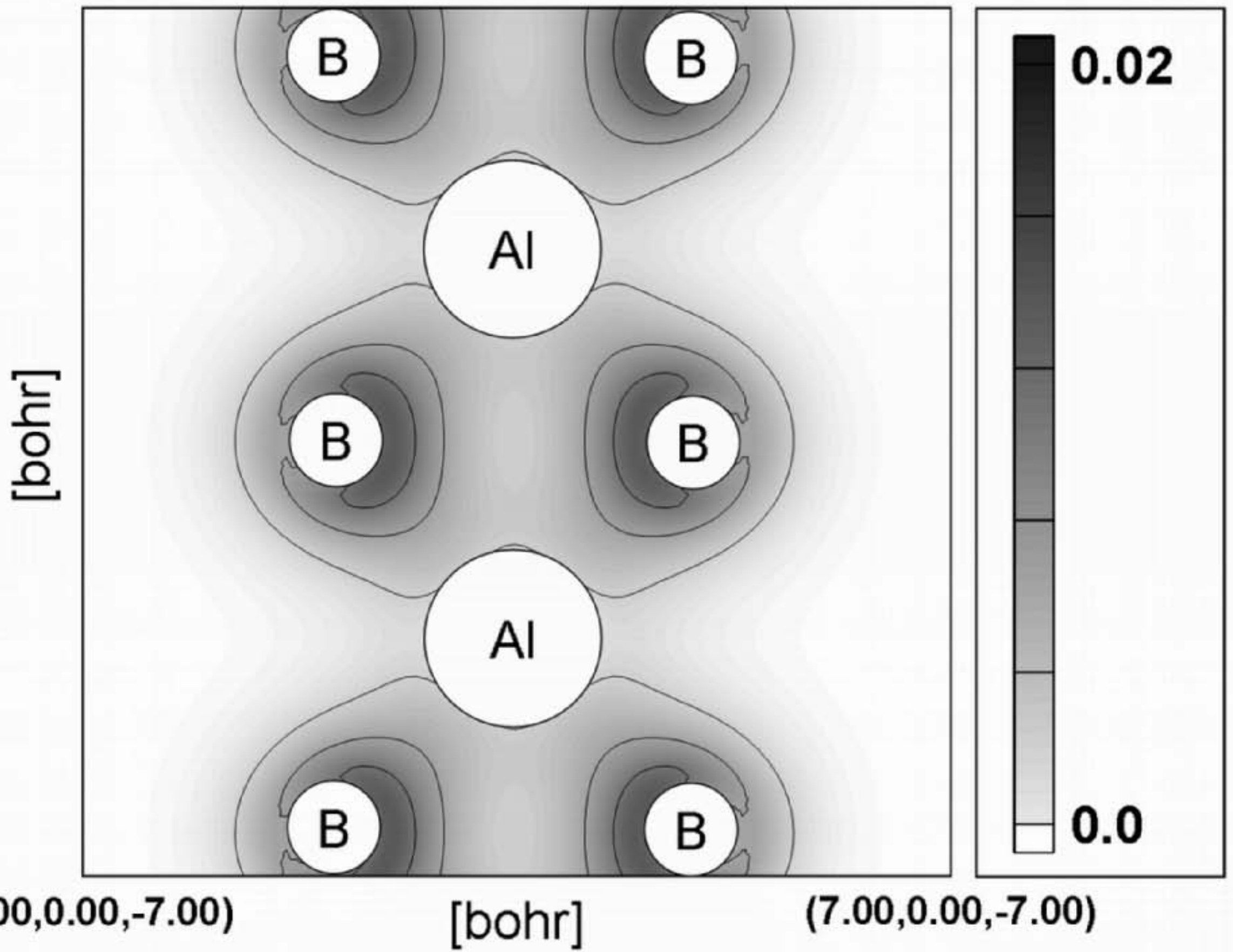

$(-7.00,0.00,-7.00)$

[bohr]

$(7.00,0.00,-7.00)$ 


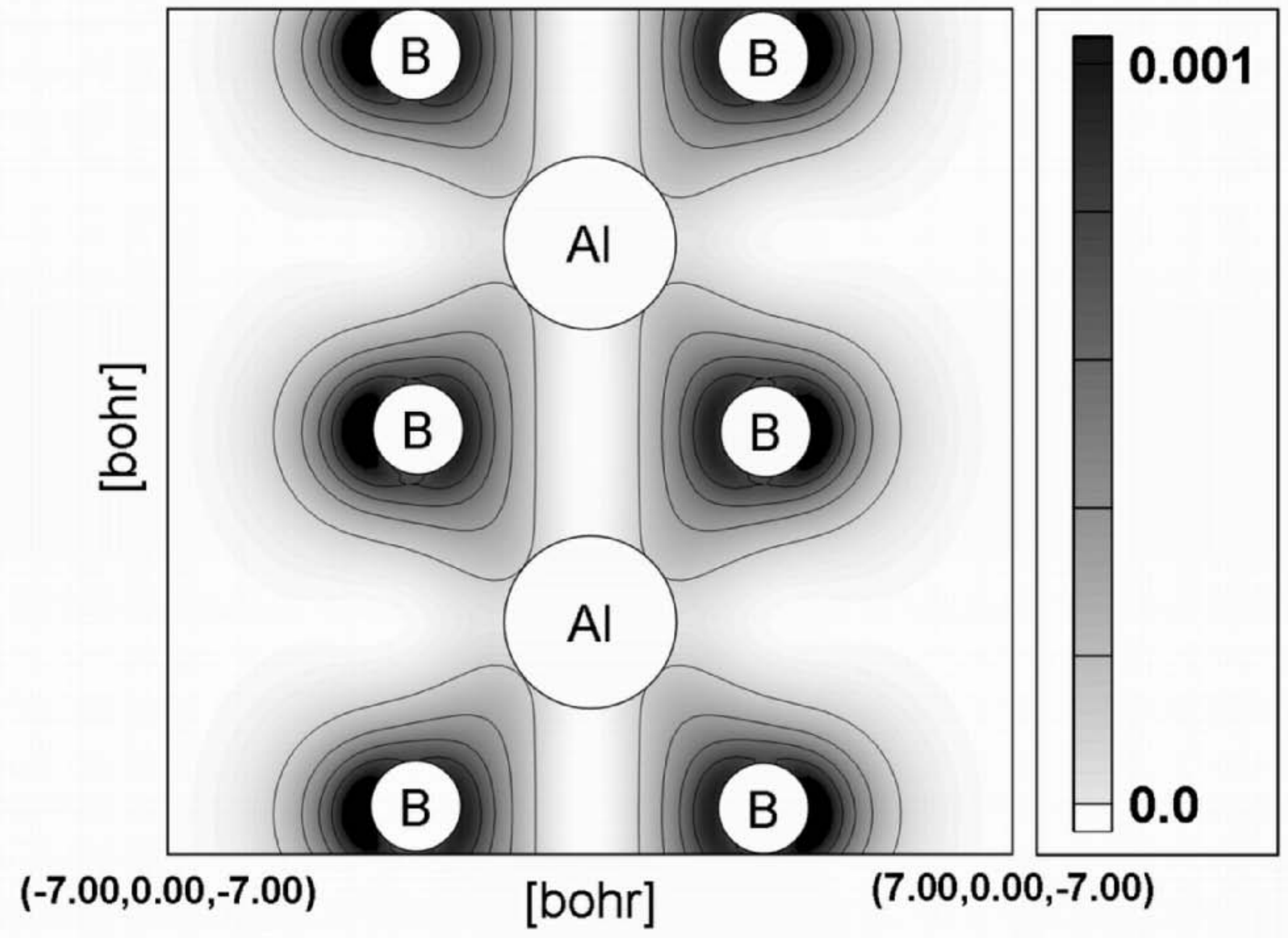




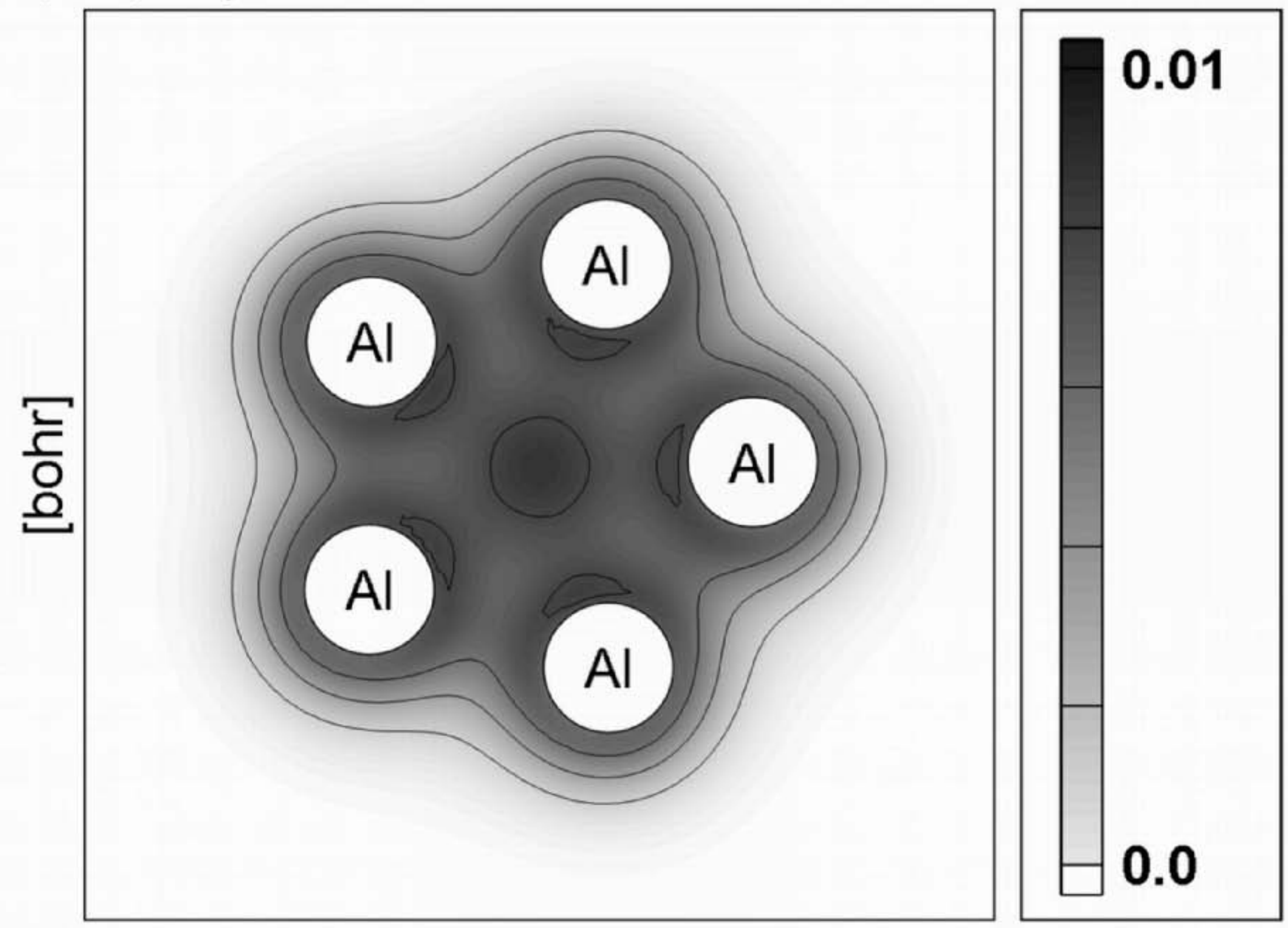




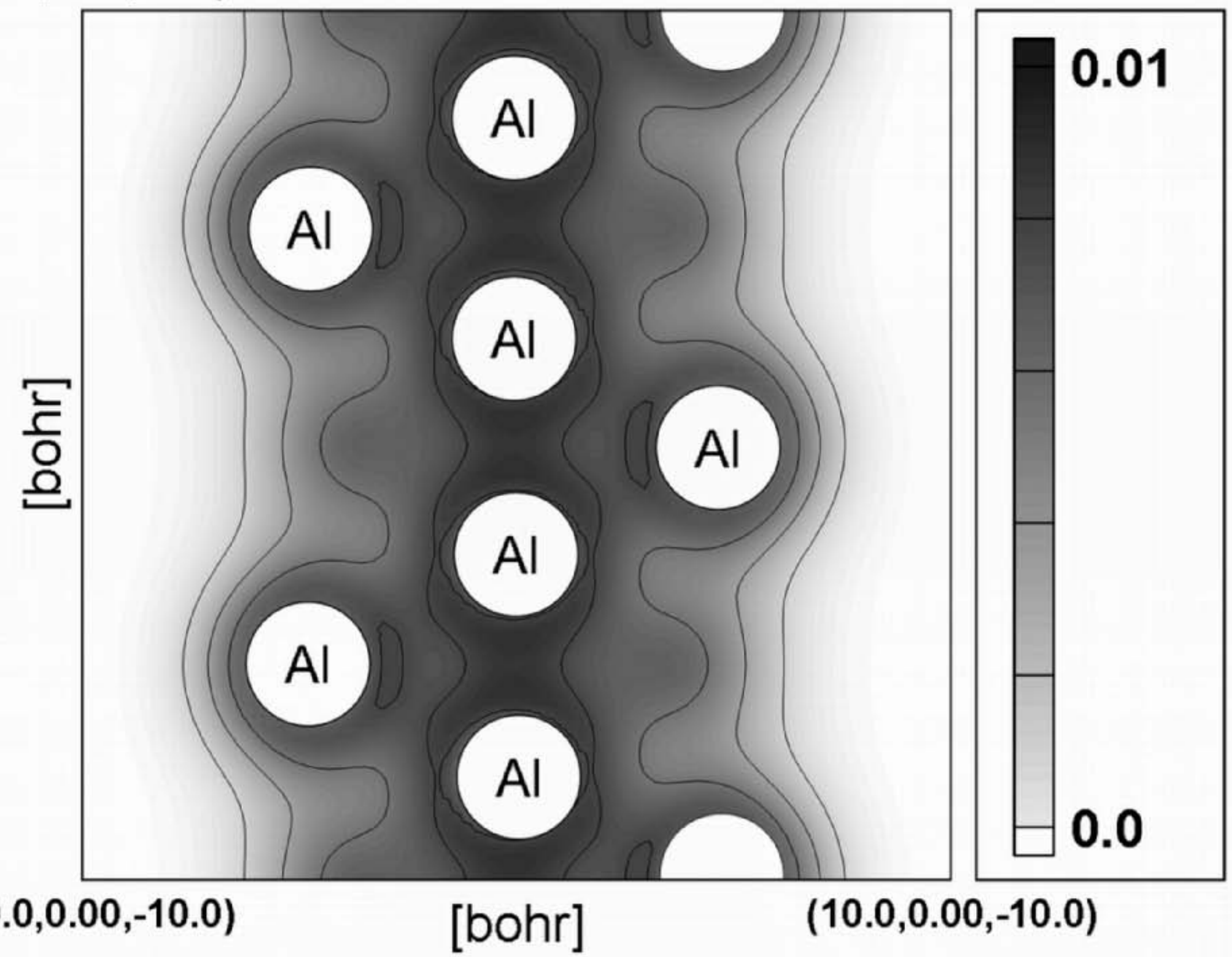




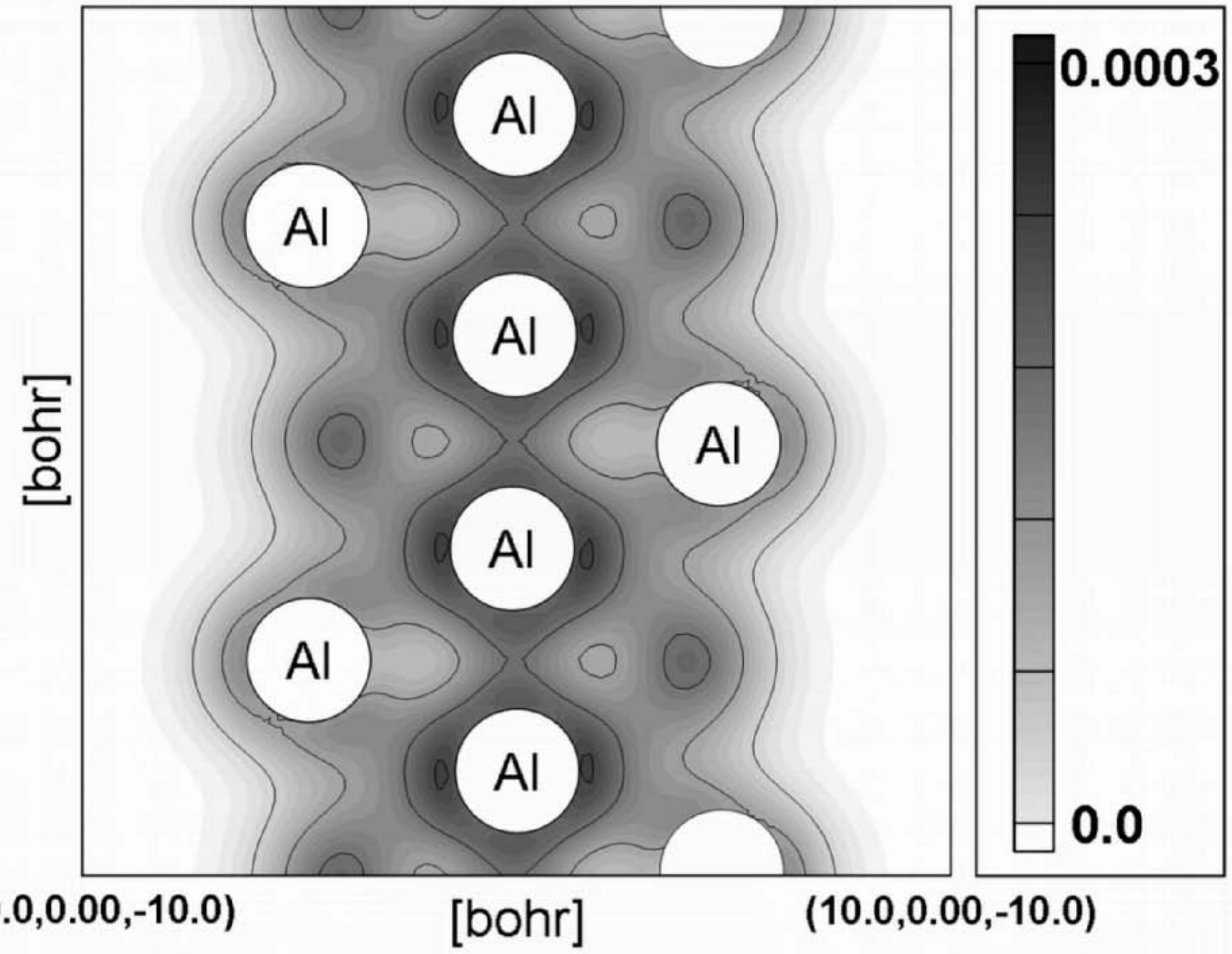




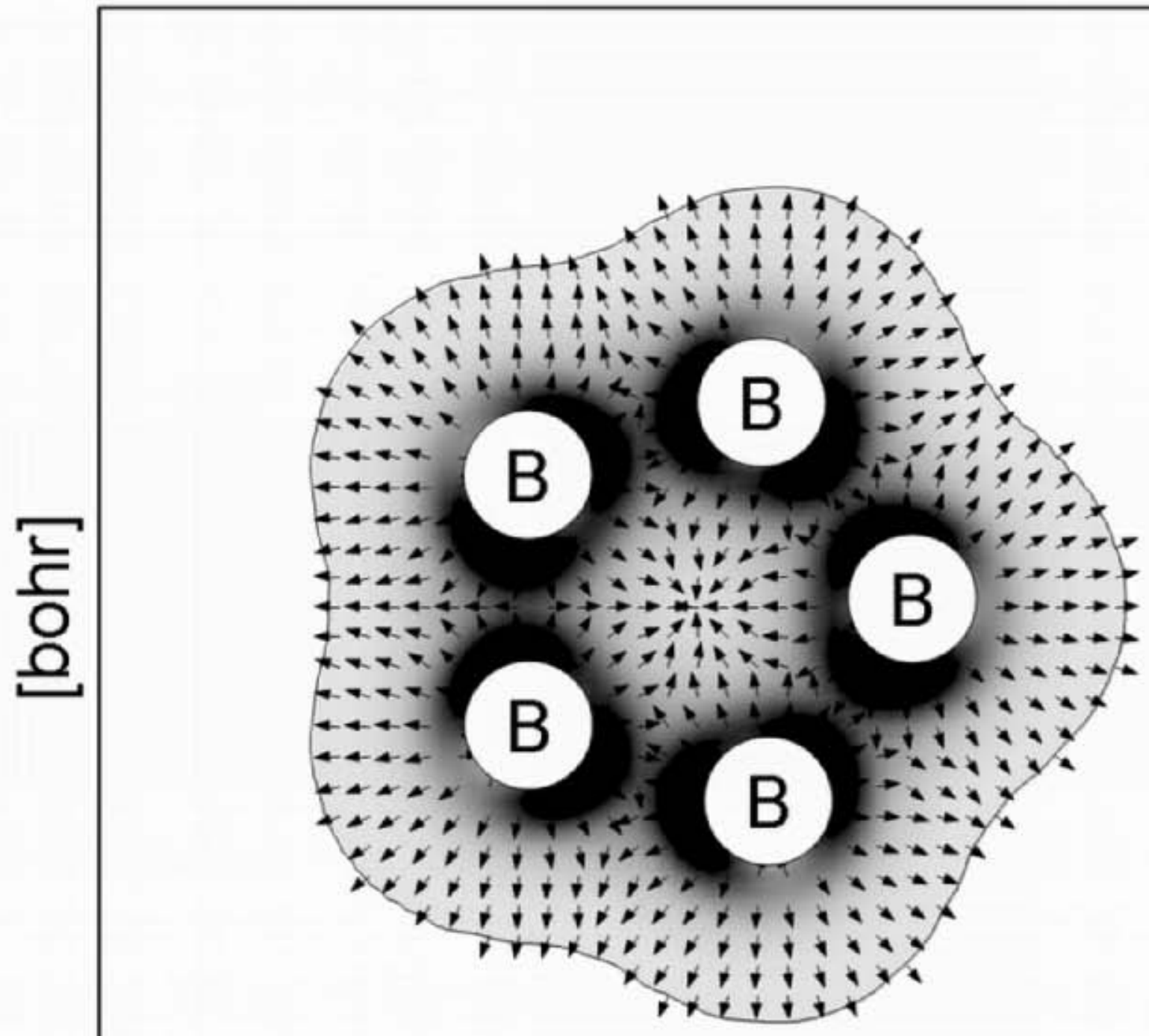

0.01 


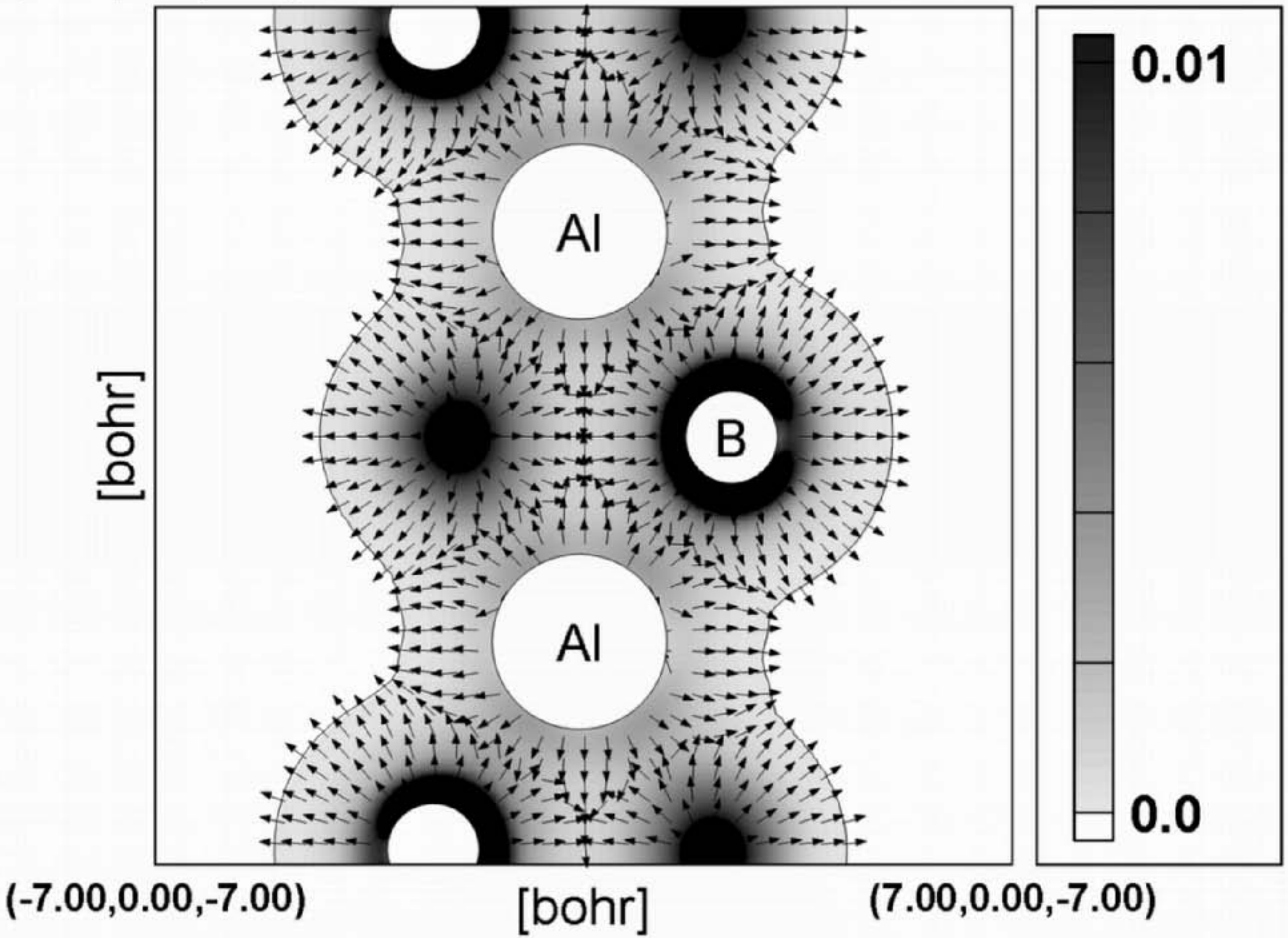





$(-7.00,0.00,-7.00)$

[bohr]

$(7.00,0.00,-7.00)$ 


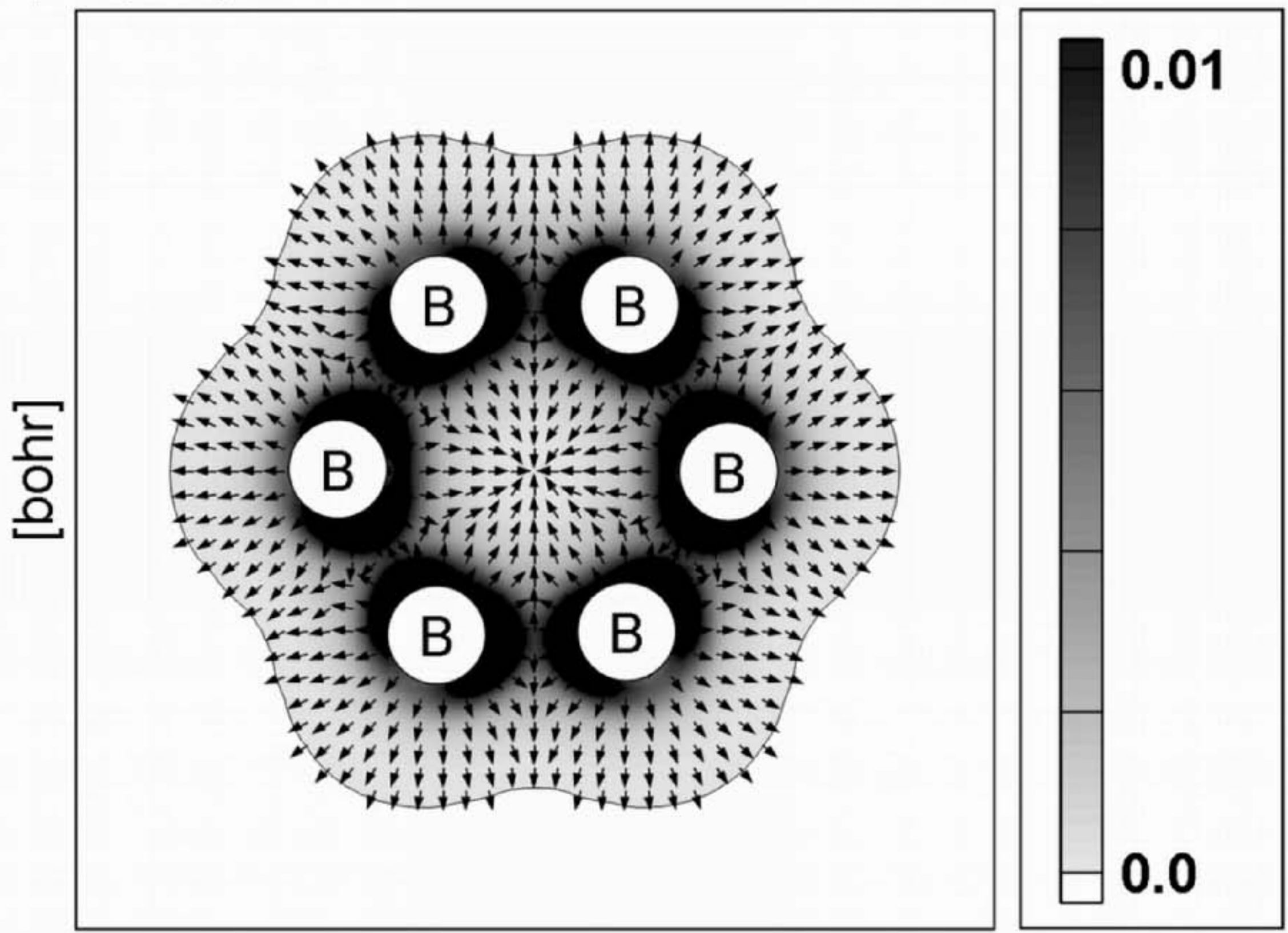



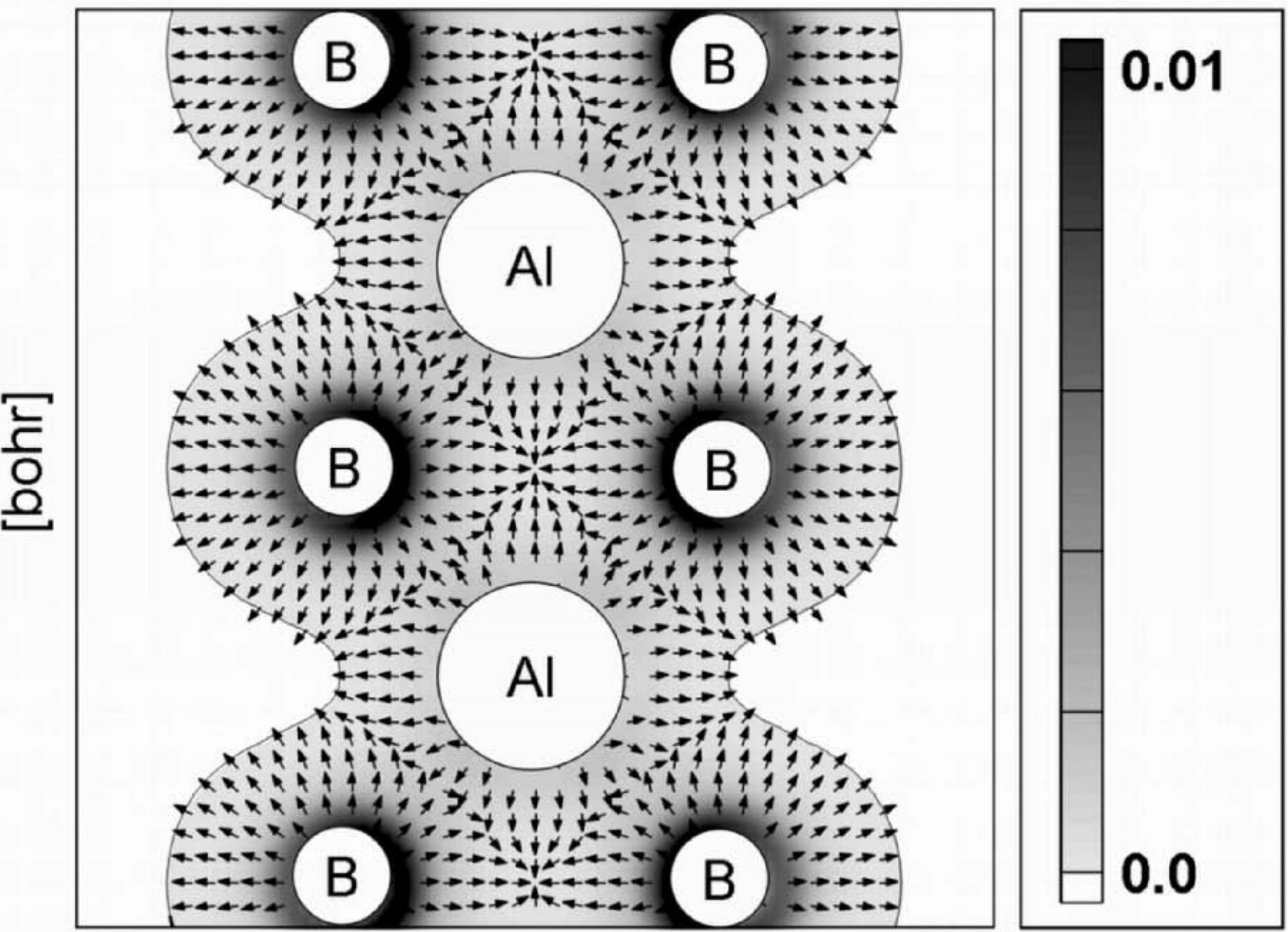

$(-7.00,0.00,-7.00)$

[bohr]

$(7.00,0.00,-7.00)$ 


\section{$(-7.00,0.00,7.00)$}
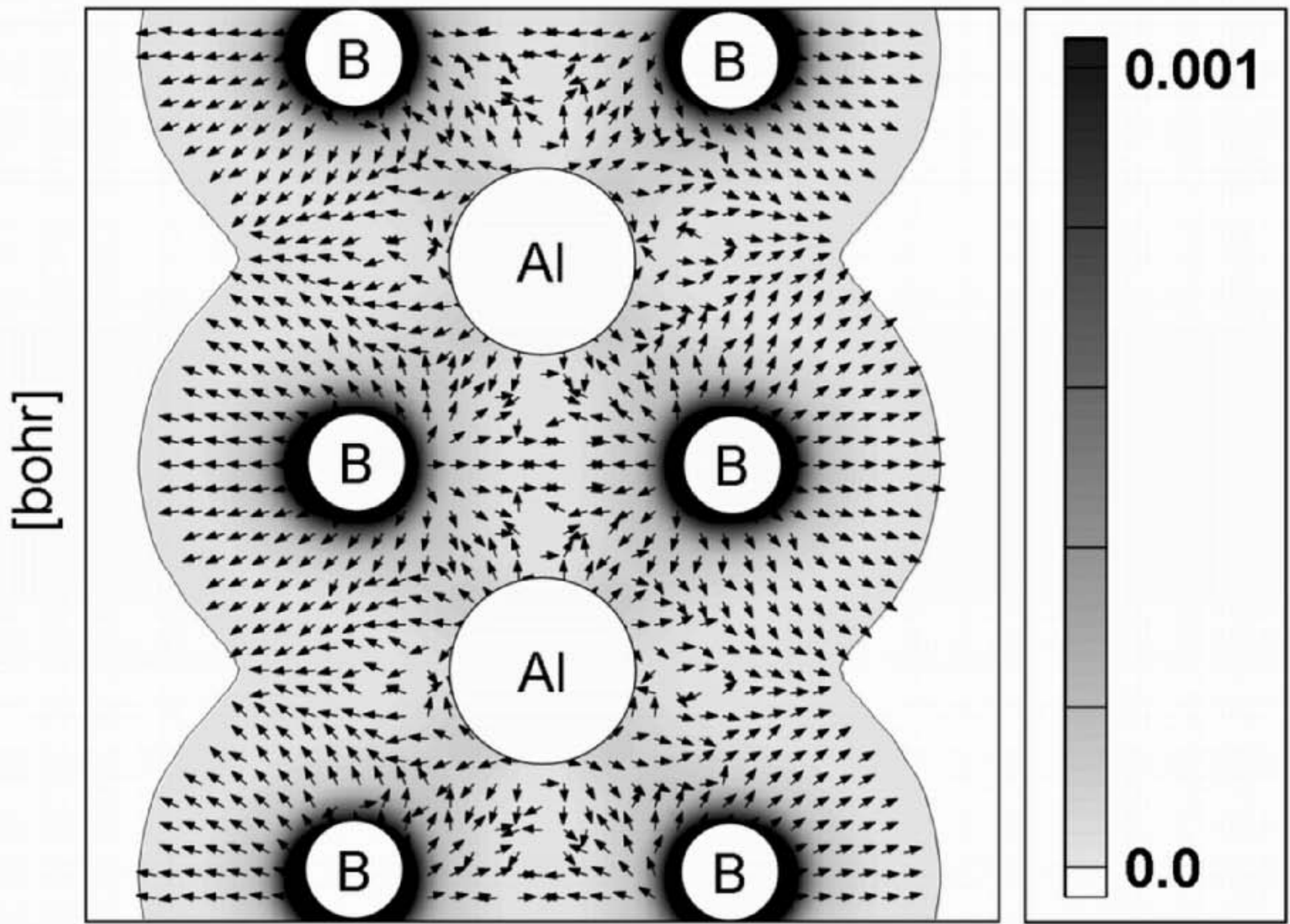

$(-7.00,0.00,-7.00)$ 

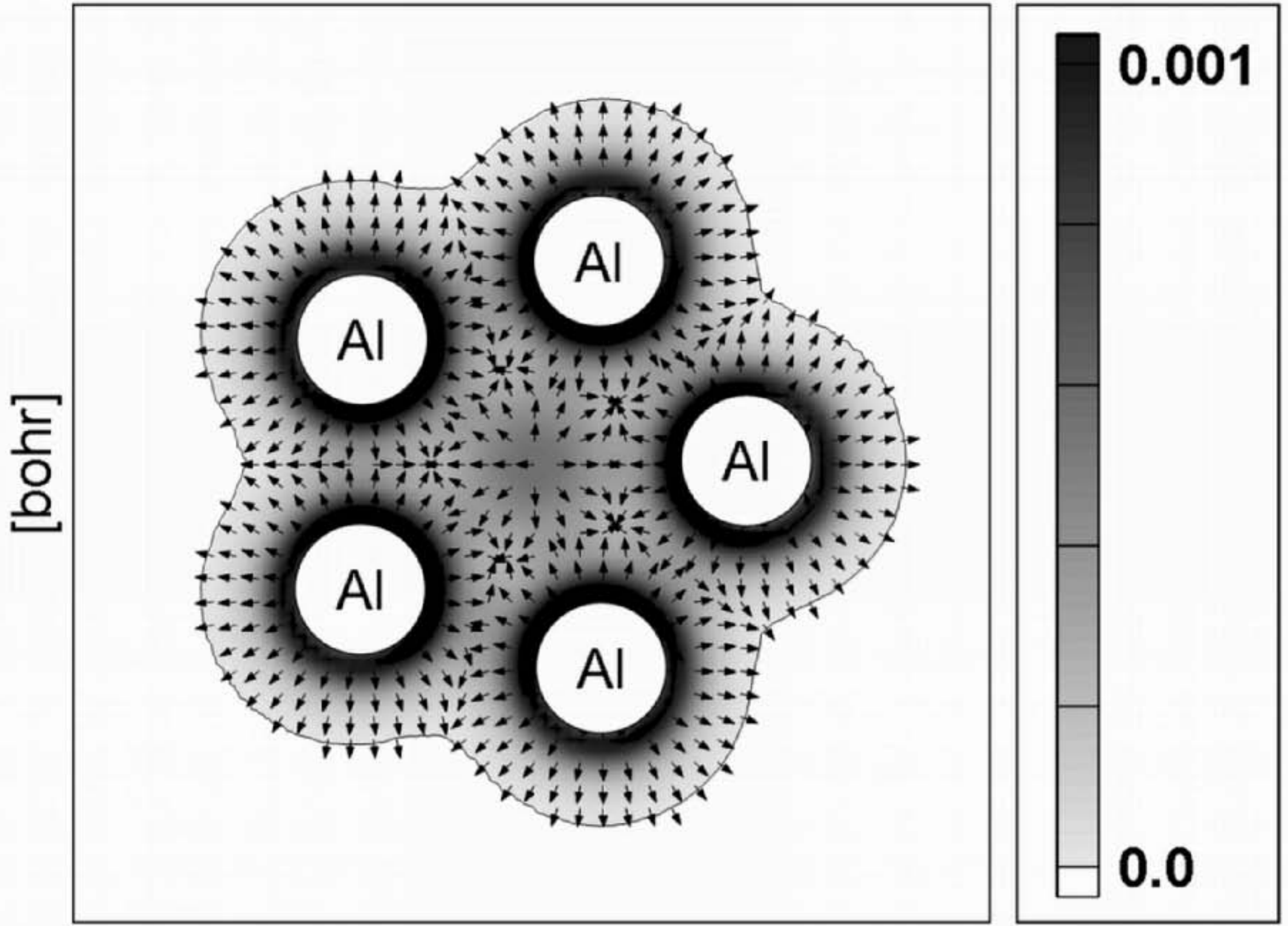


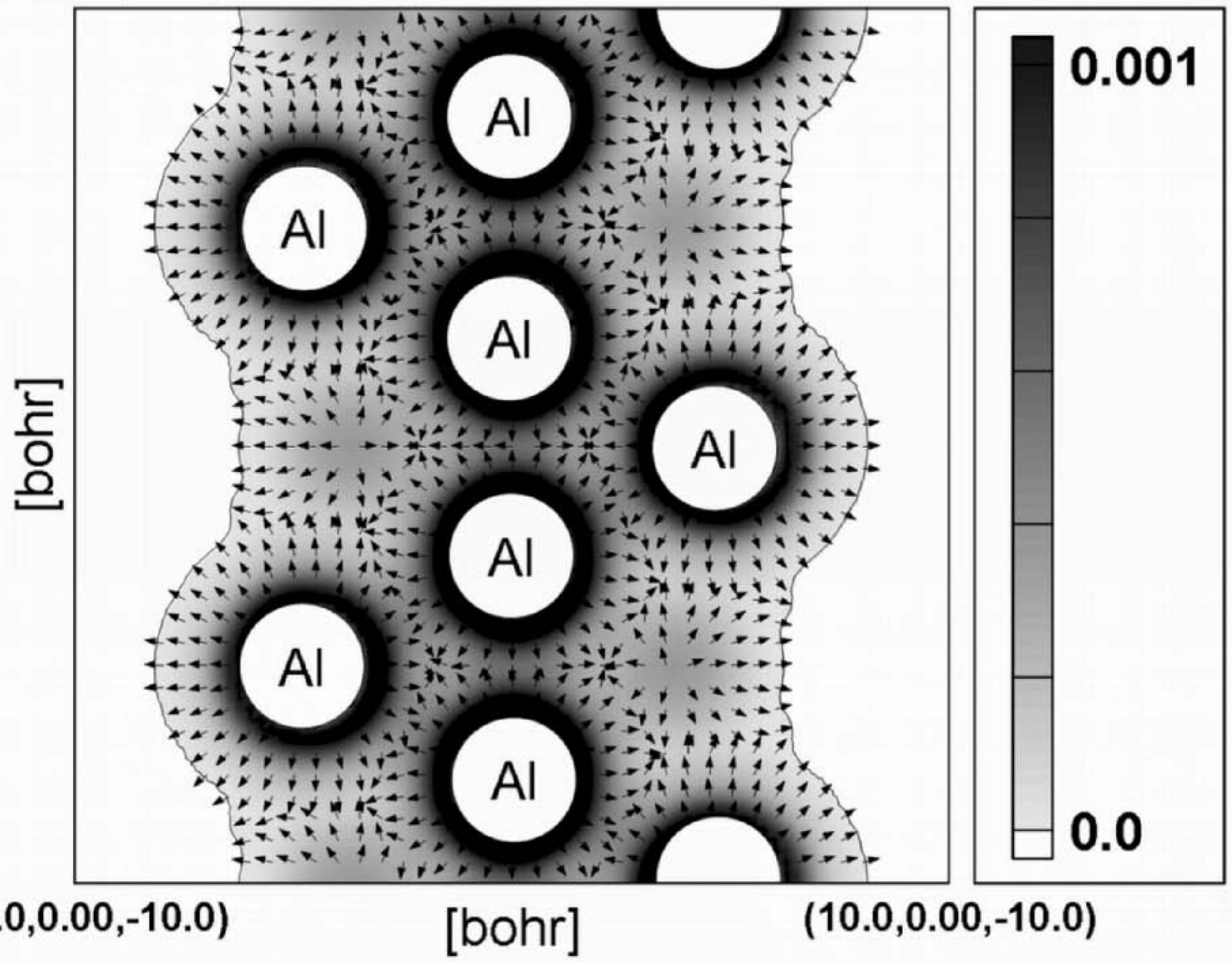




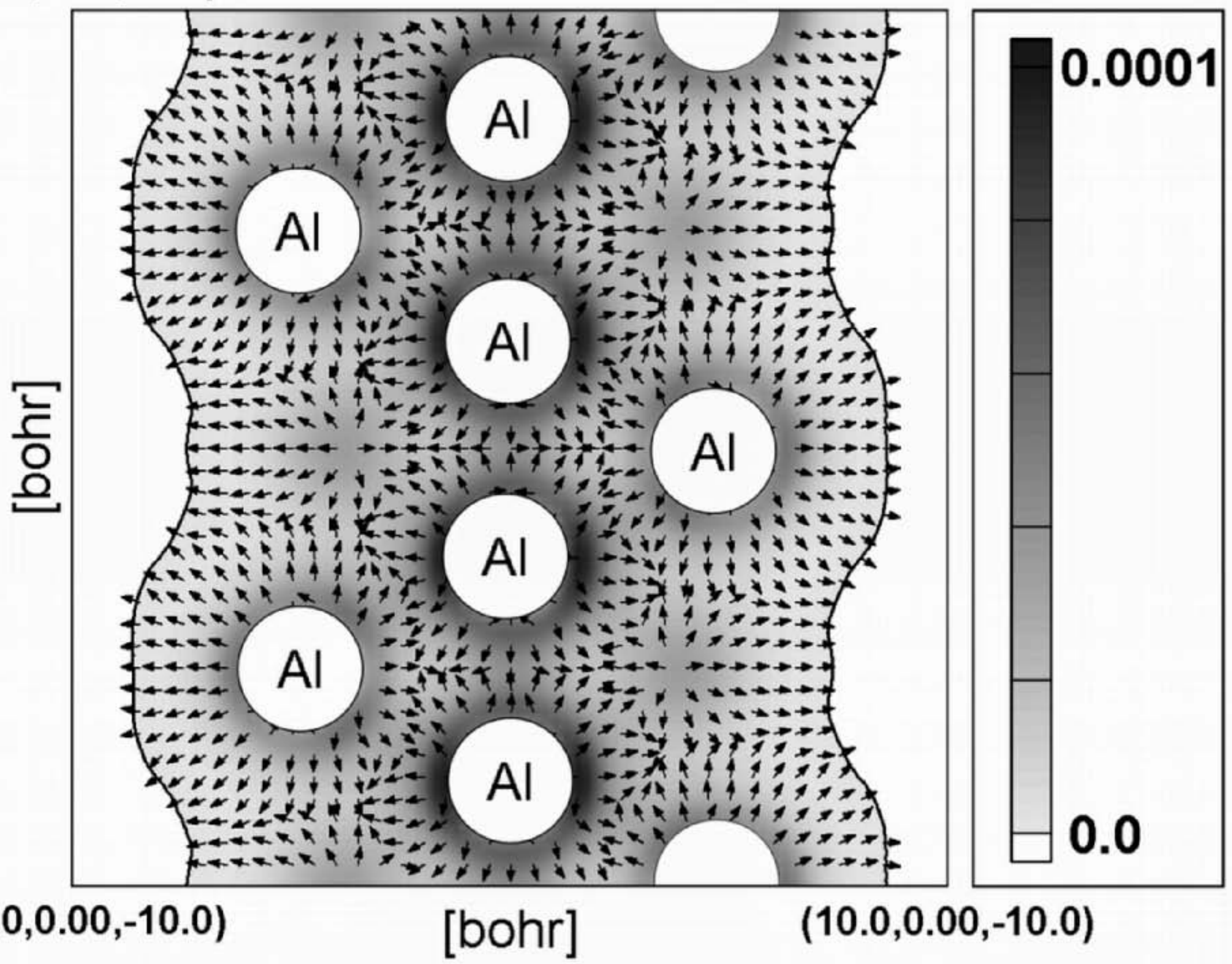

$(-10.0,0.00,-10.0)$ 
$(-7.00,7.00,0.00)$

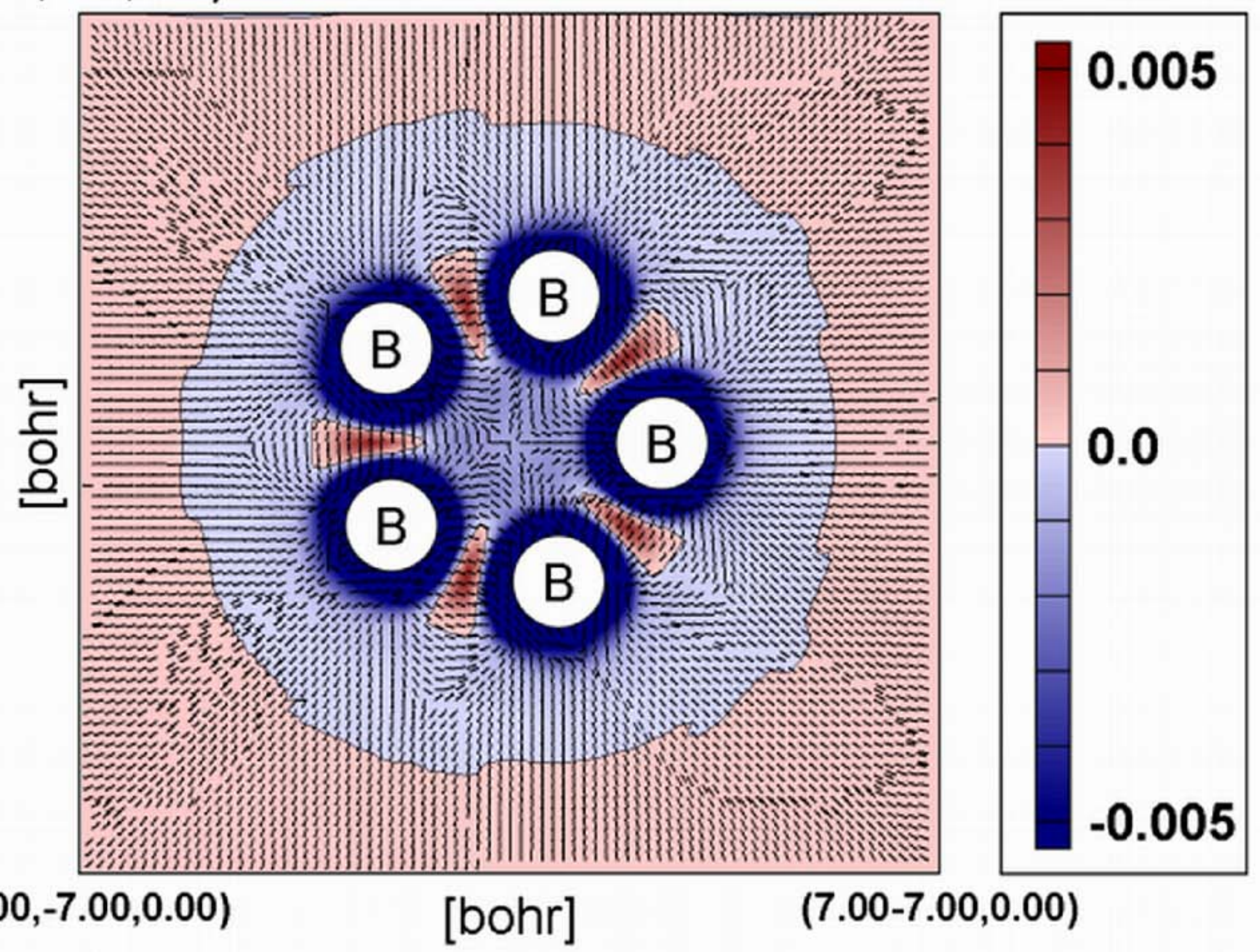


$(-7.00,0.00,7.00)$
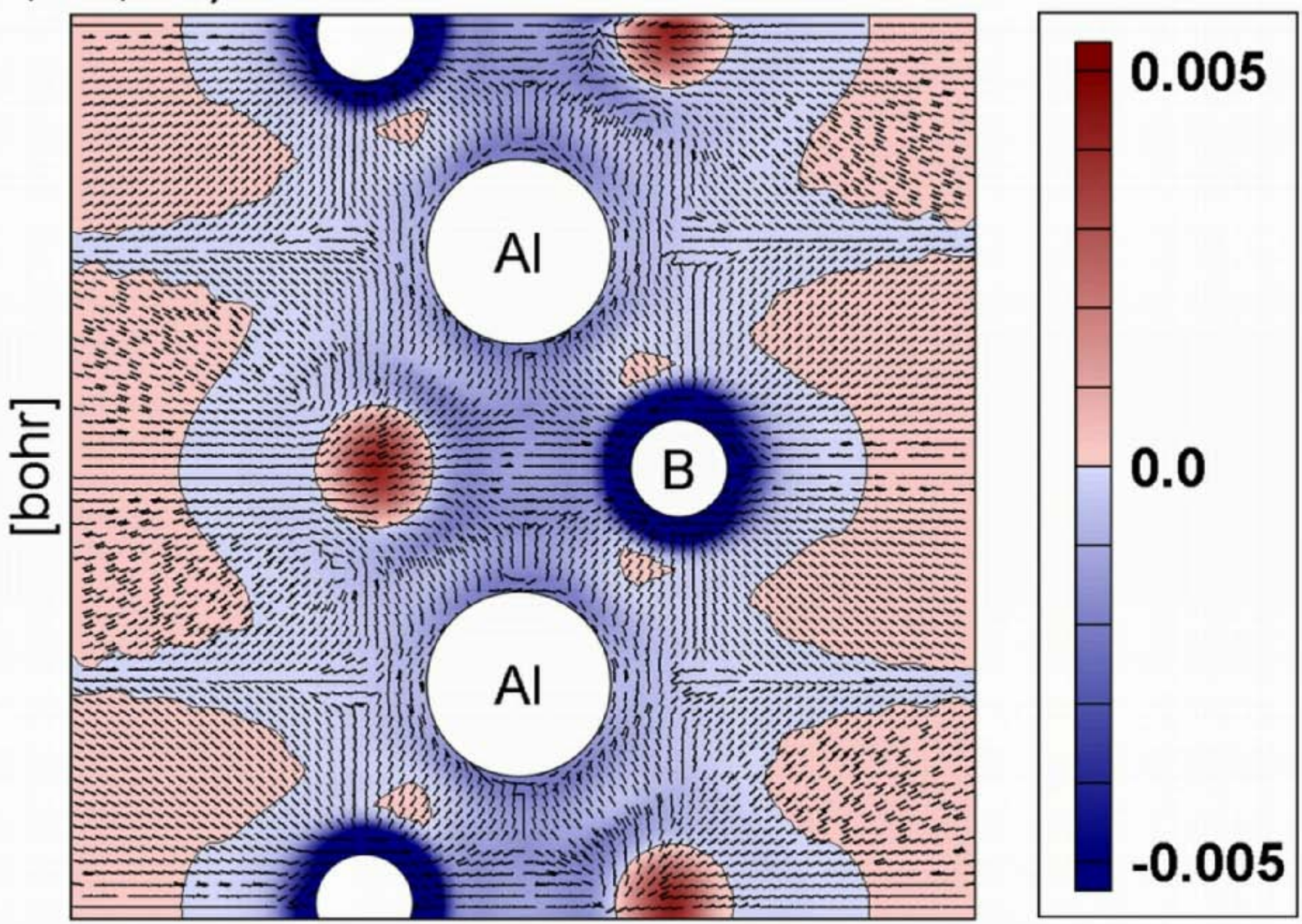

$(-7.00,0.00,-7.00)$

[bohr]

$(7.00,0.00,-7.00)$ 


\section{$(-7.00,7.00,0.00)$}

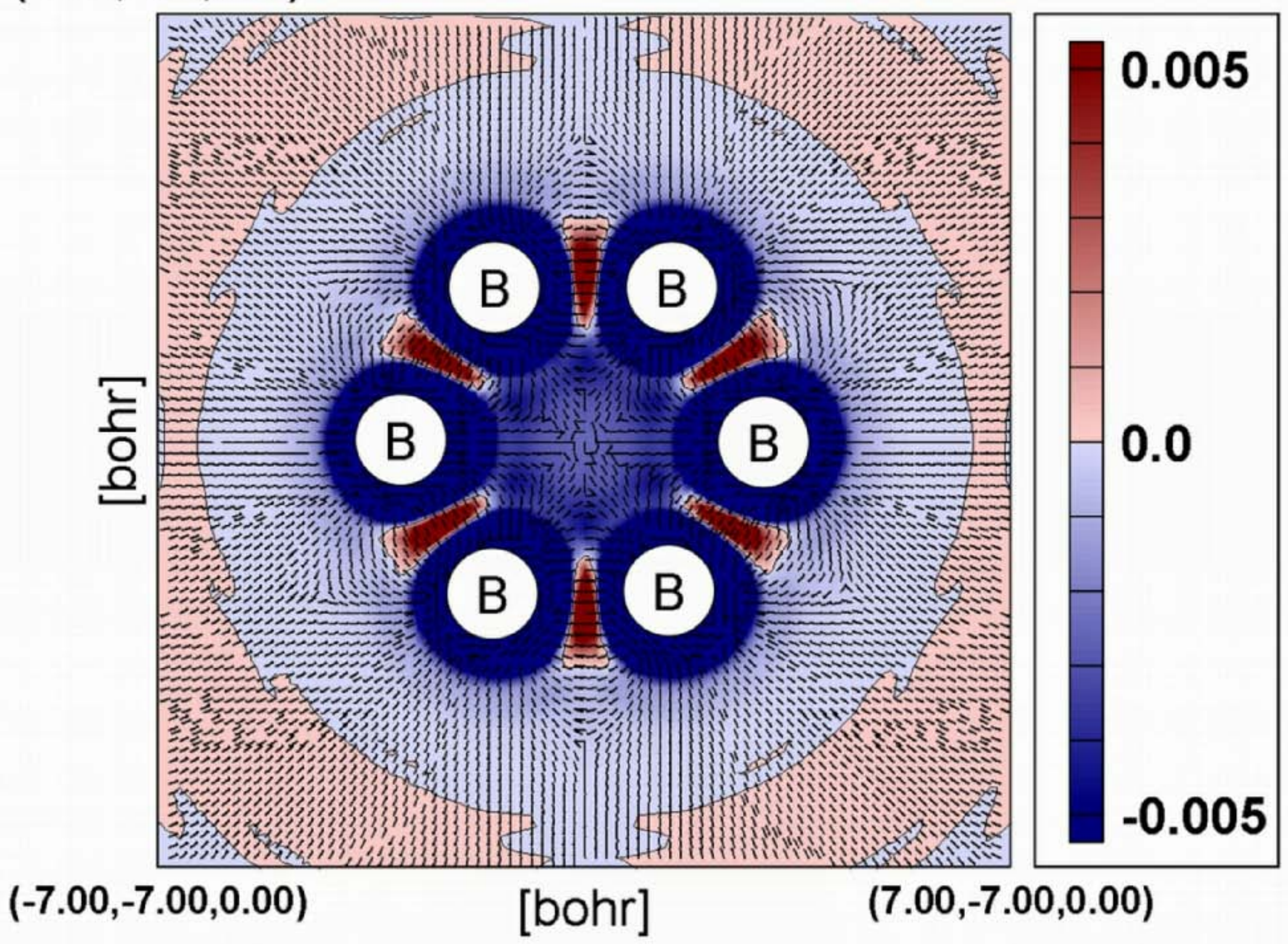




\section{$(-7.00,0.00,7.00)$}

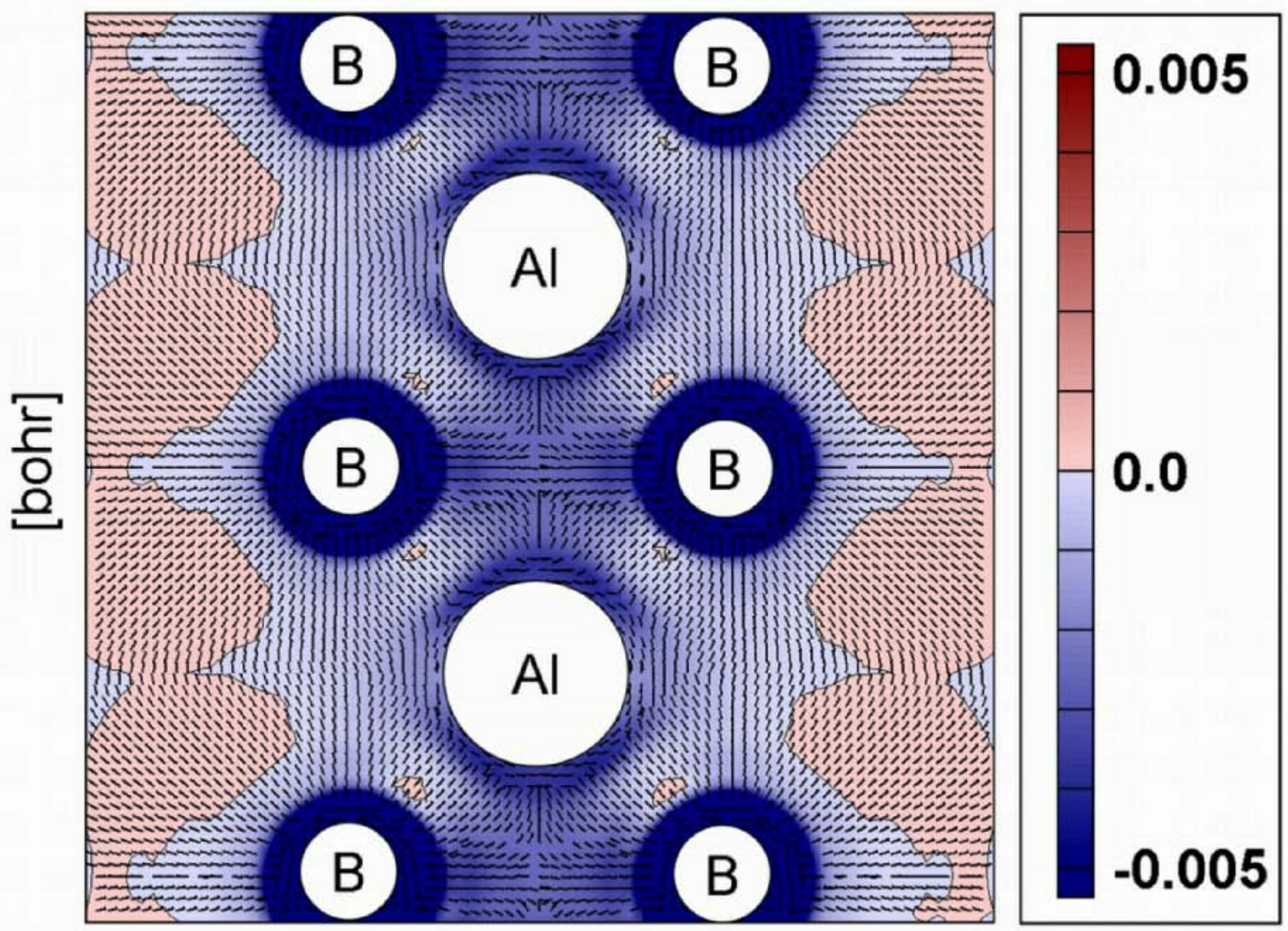

$(-7.00,0.00,-7.00)$

[bohr]

$(7.00,0.00,-7.00)$ 



$(-10.0,-10.0,0.00)$

[bohr]

$(10.0,-10.0,0.00)$ 


\section{$(-10.0,0.00,10.0)$}

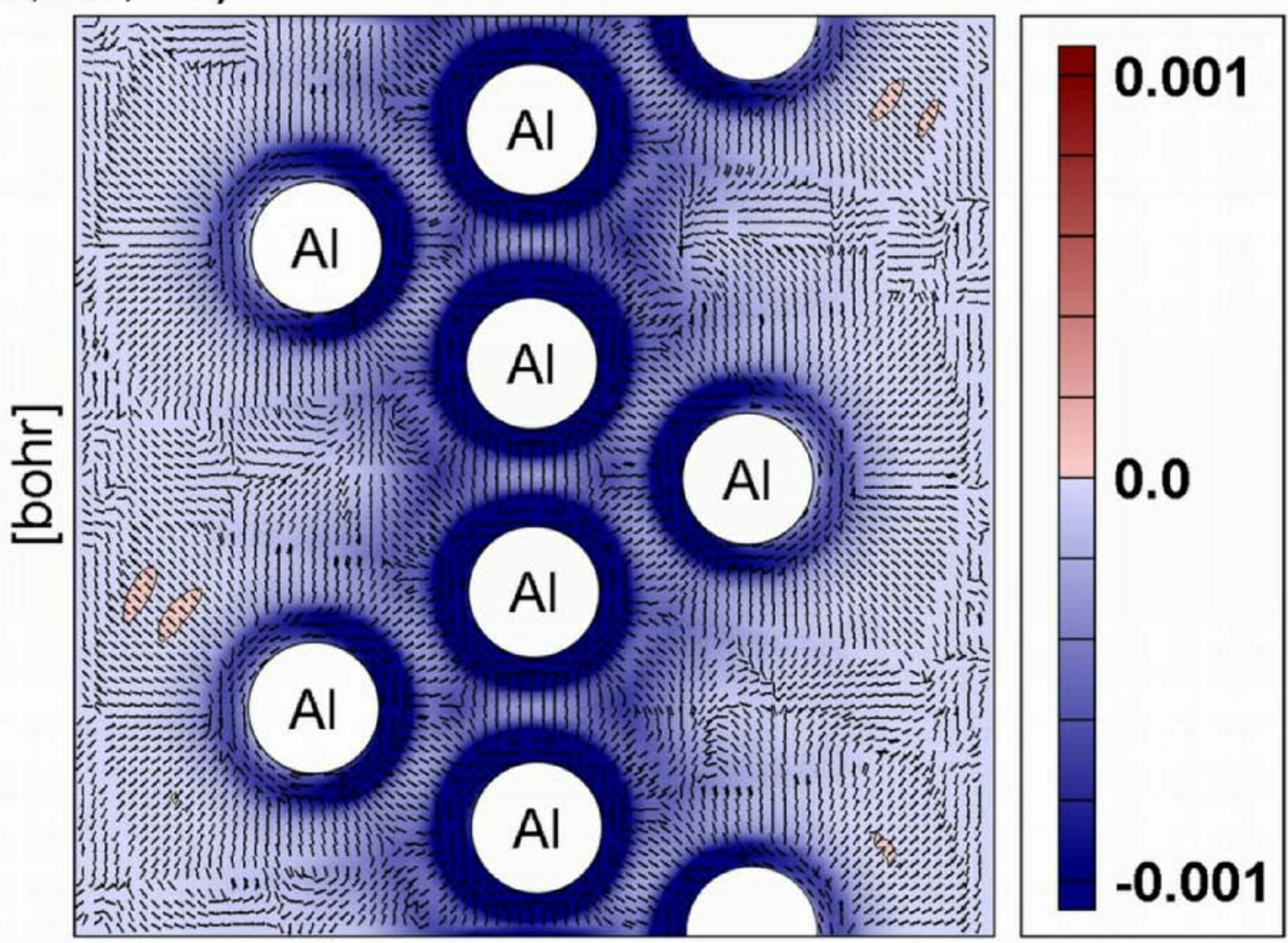

$(-10.0,0.00,-10.0)$

[bohr]

$(10.0,0.00,-10.0)$ 

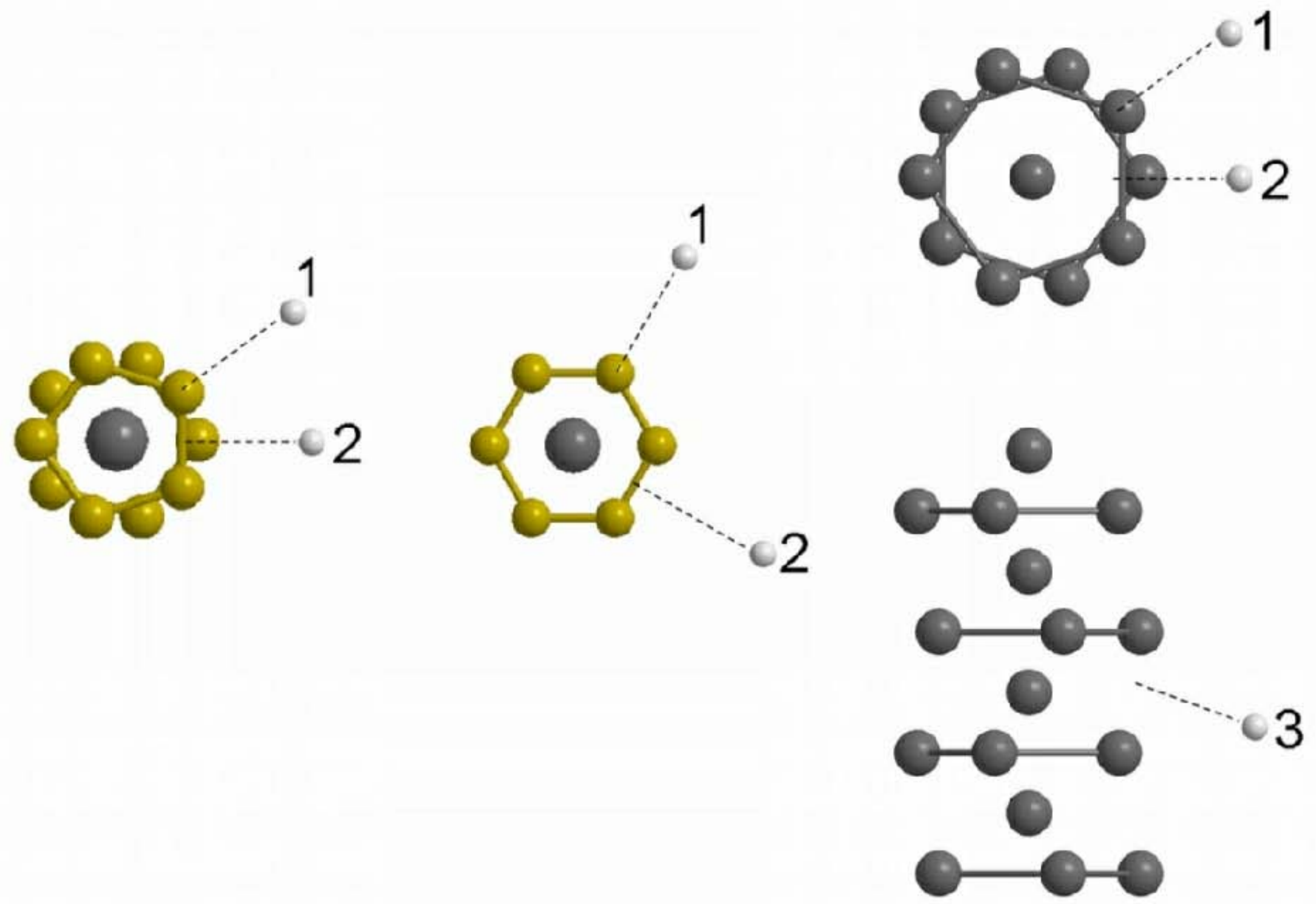

b
C 

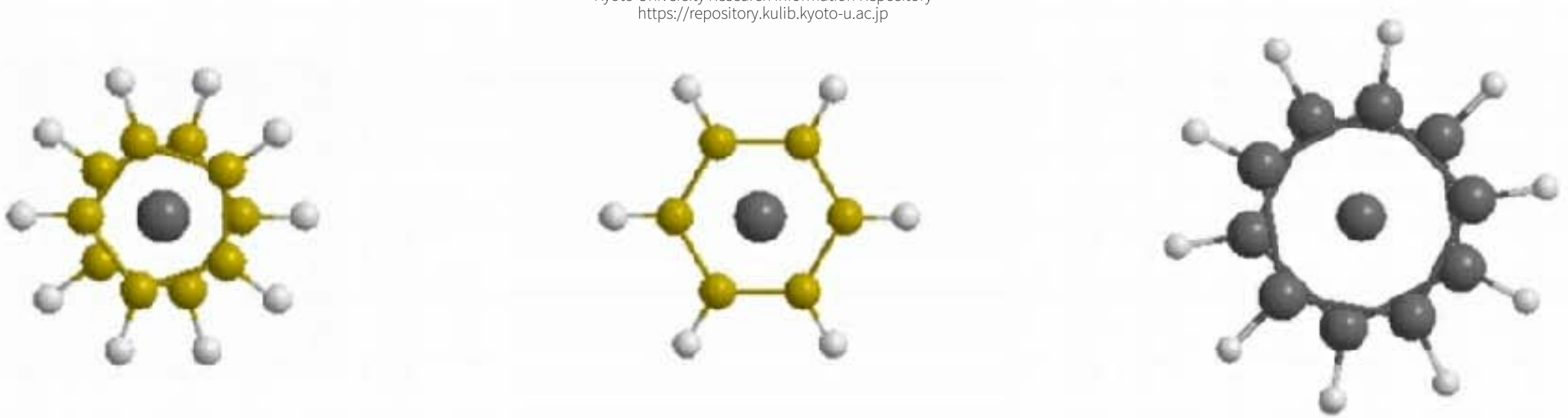

KURENAII 红
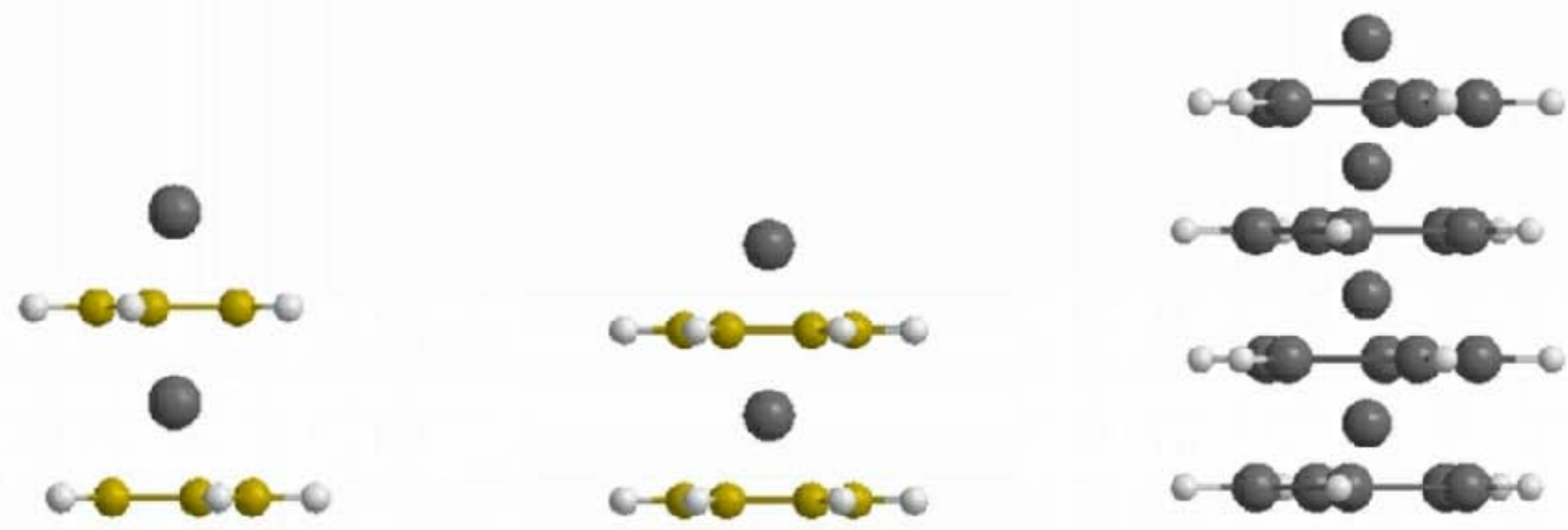

a

b

C 


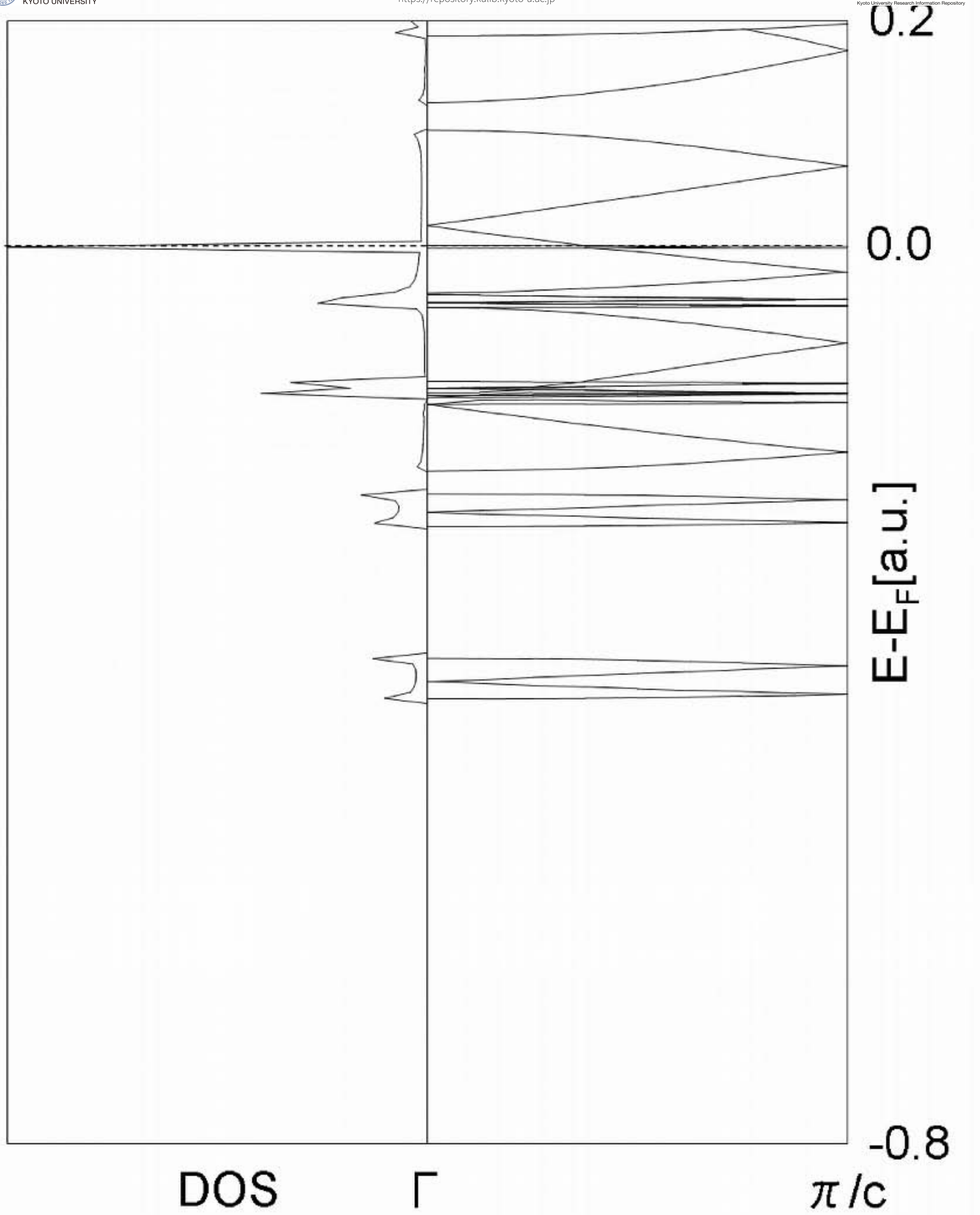




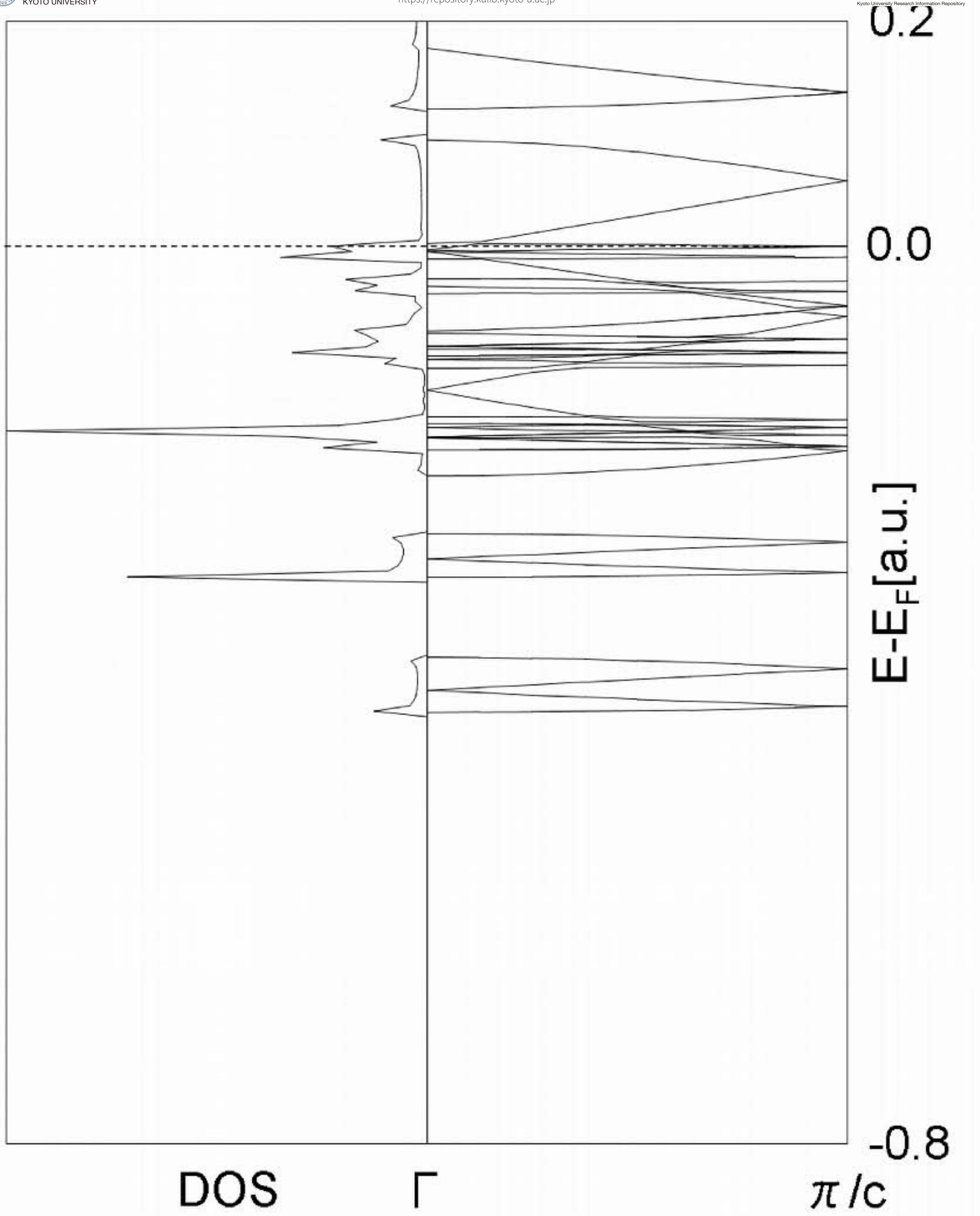




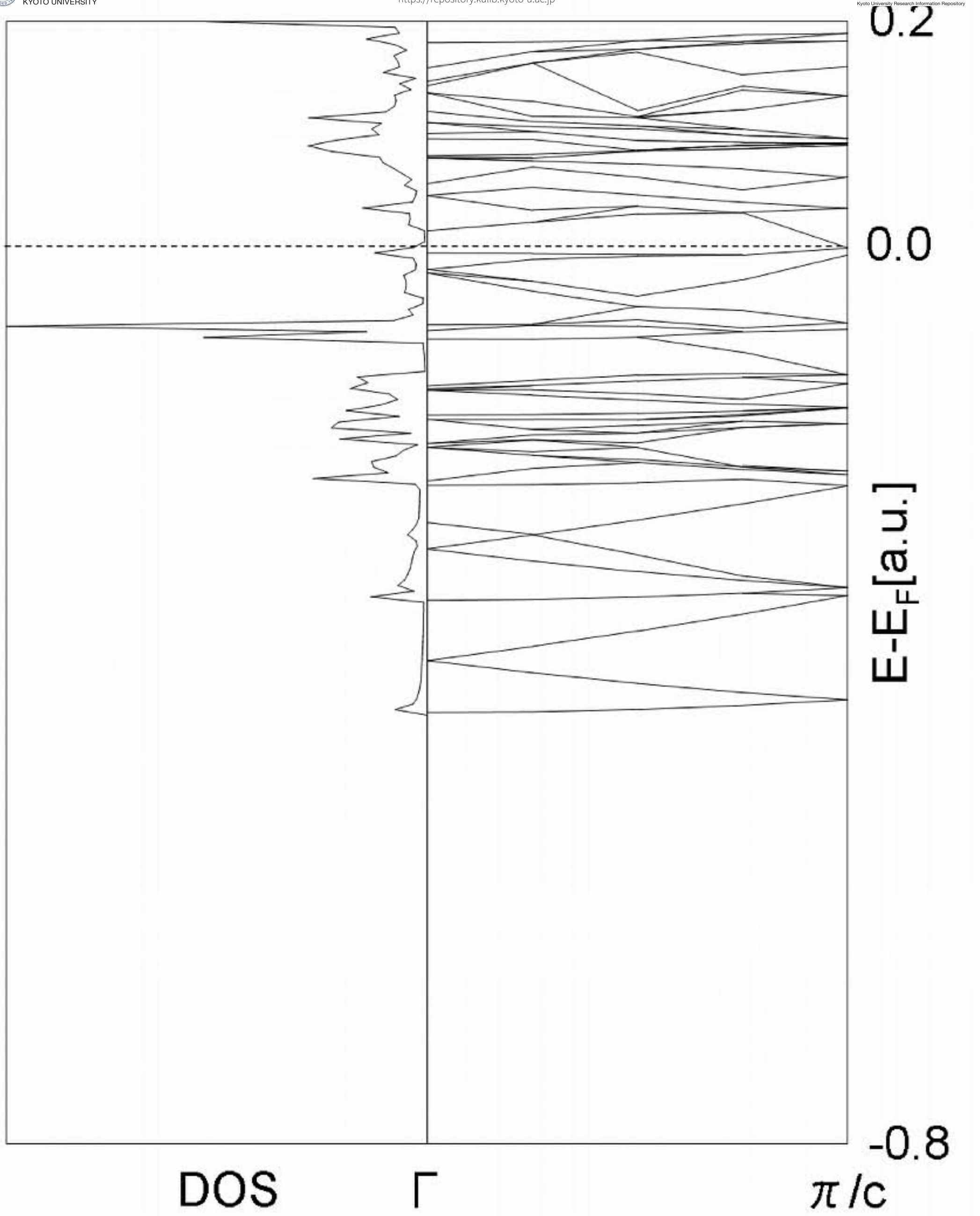




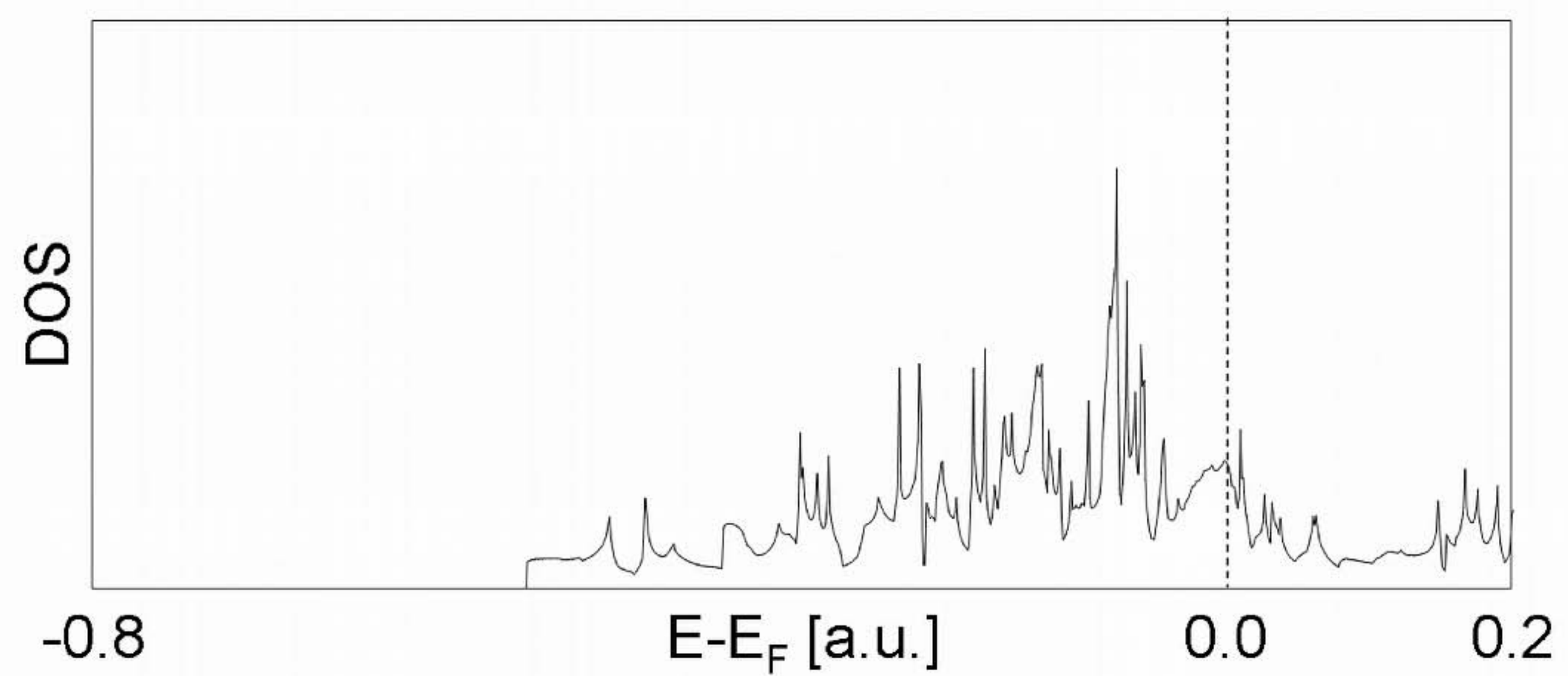




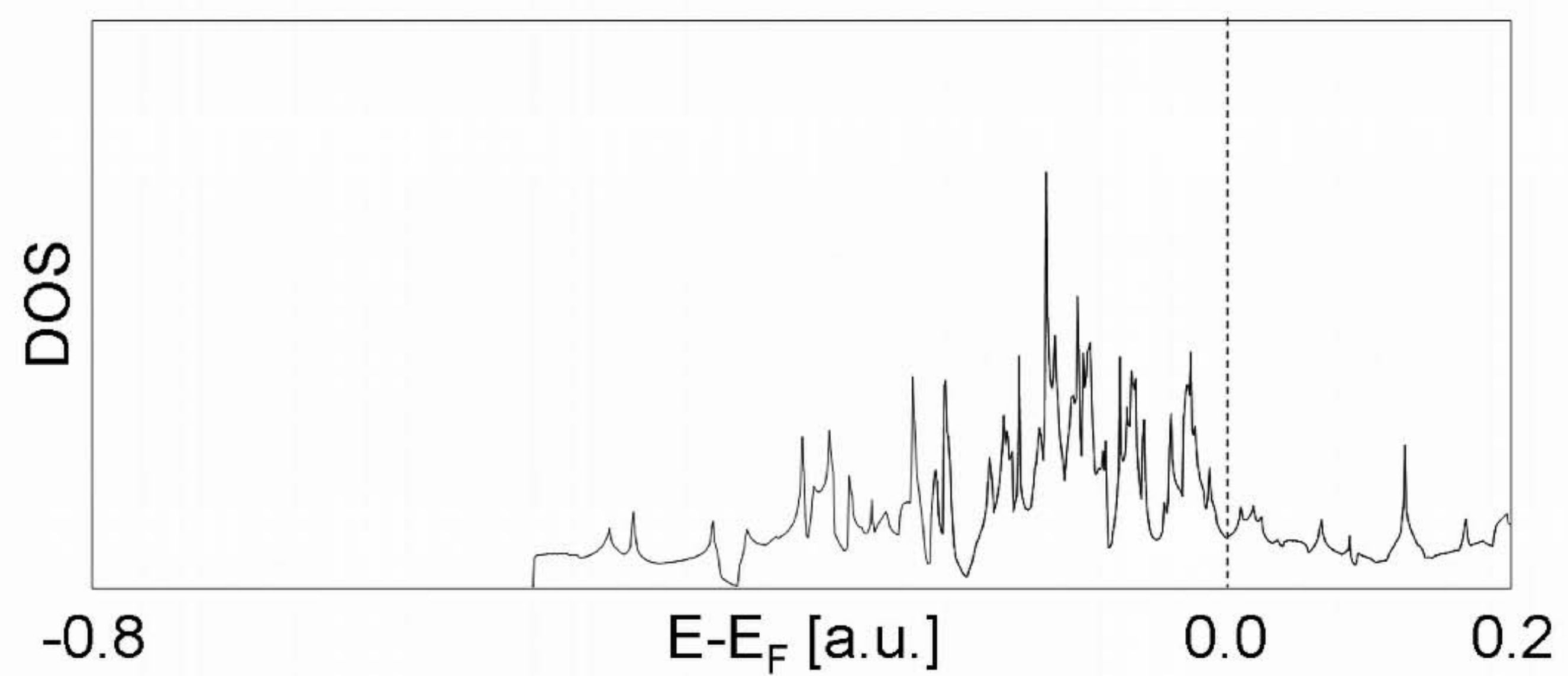




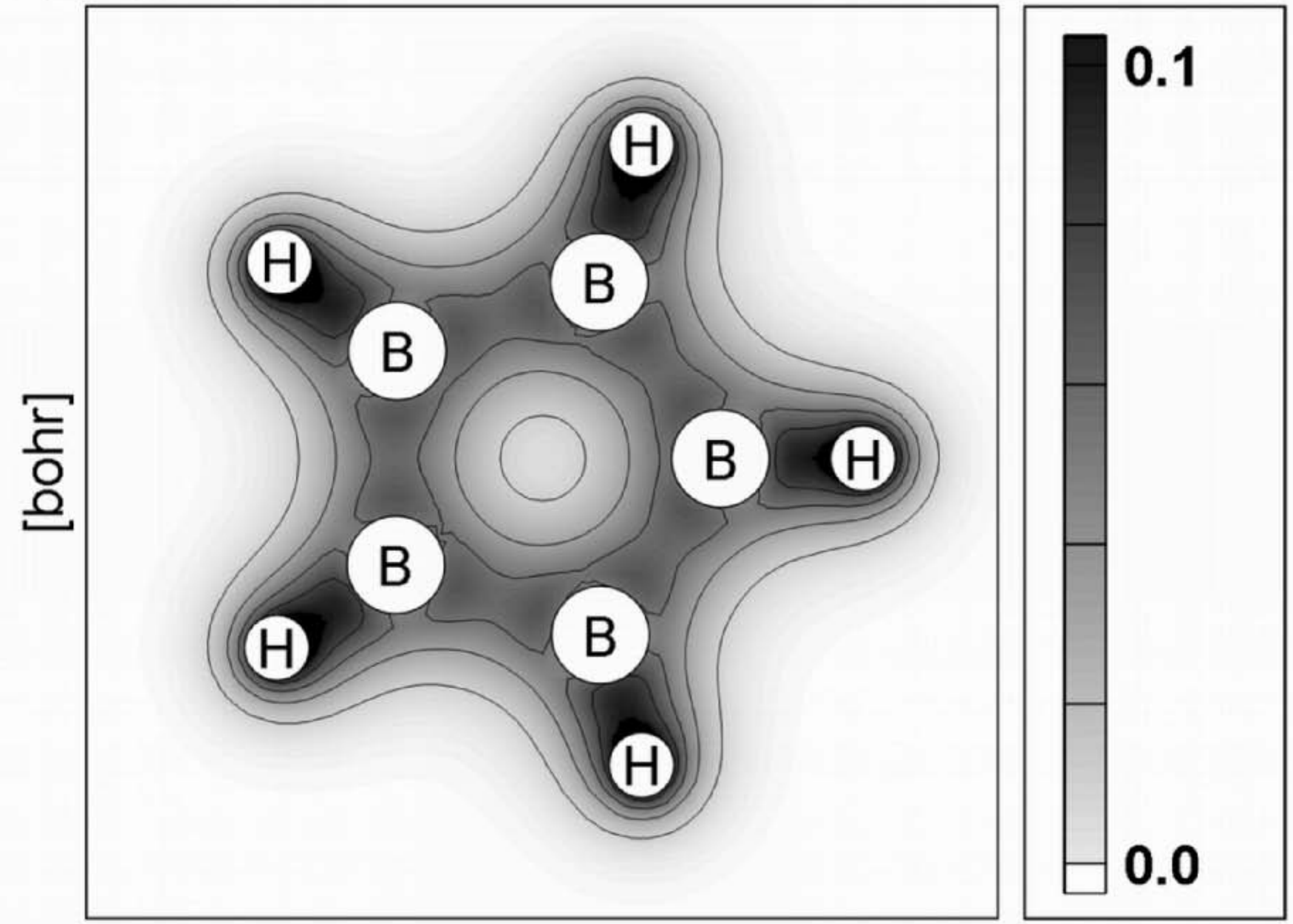




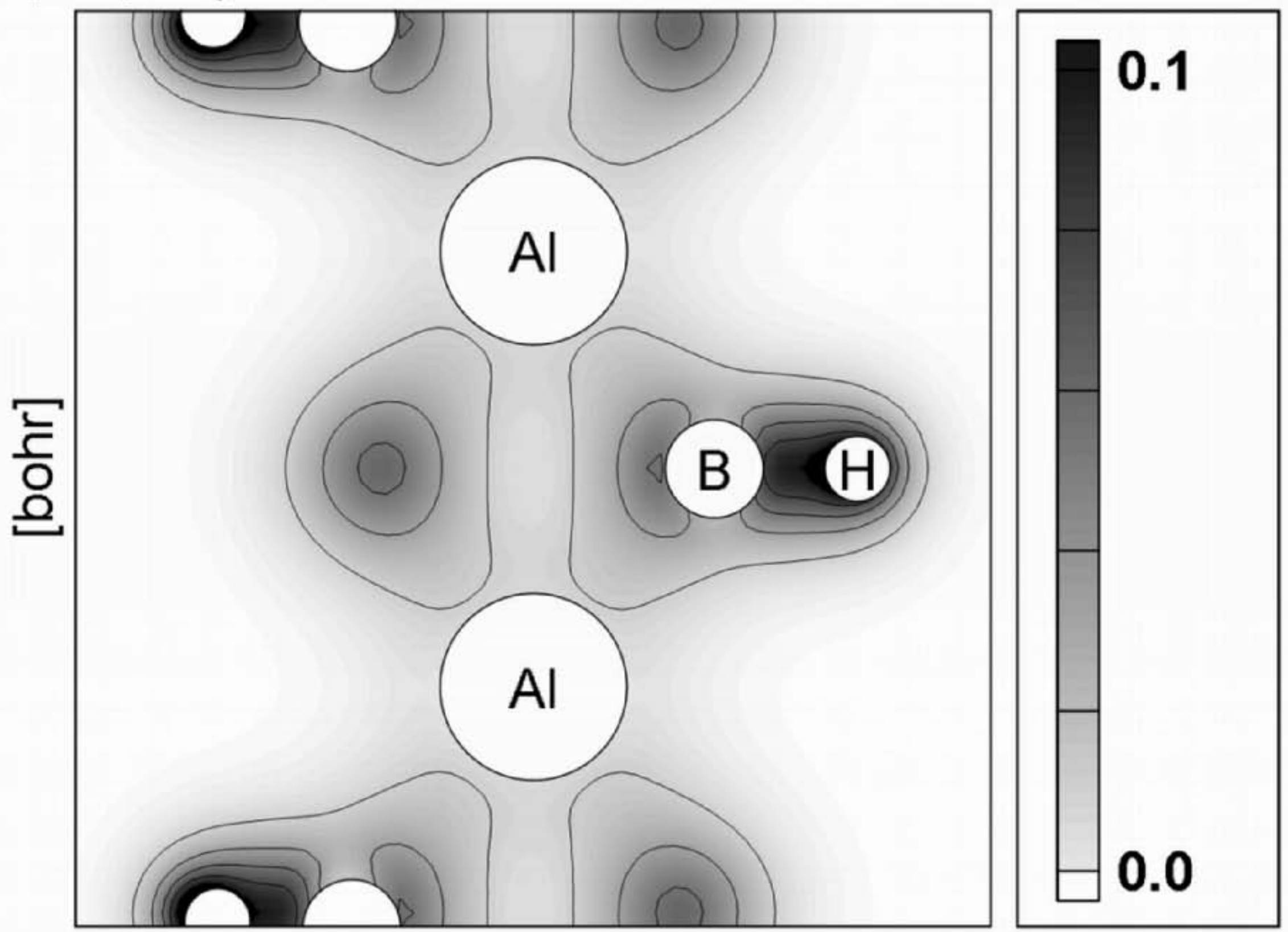




\section{$(-7.00,0.00,7.00)$}

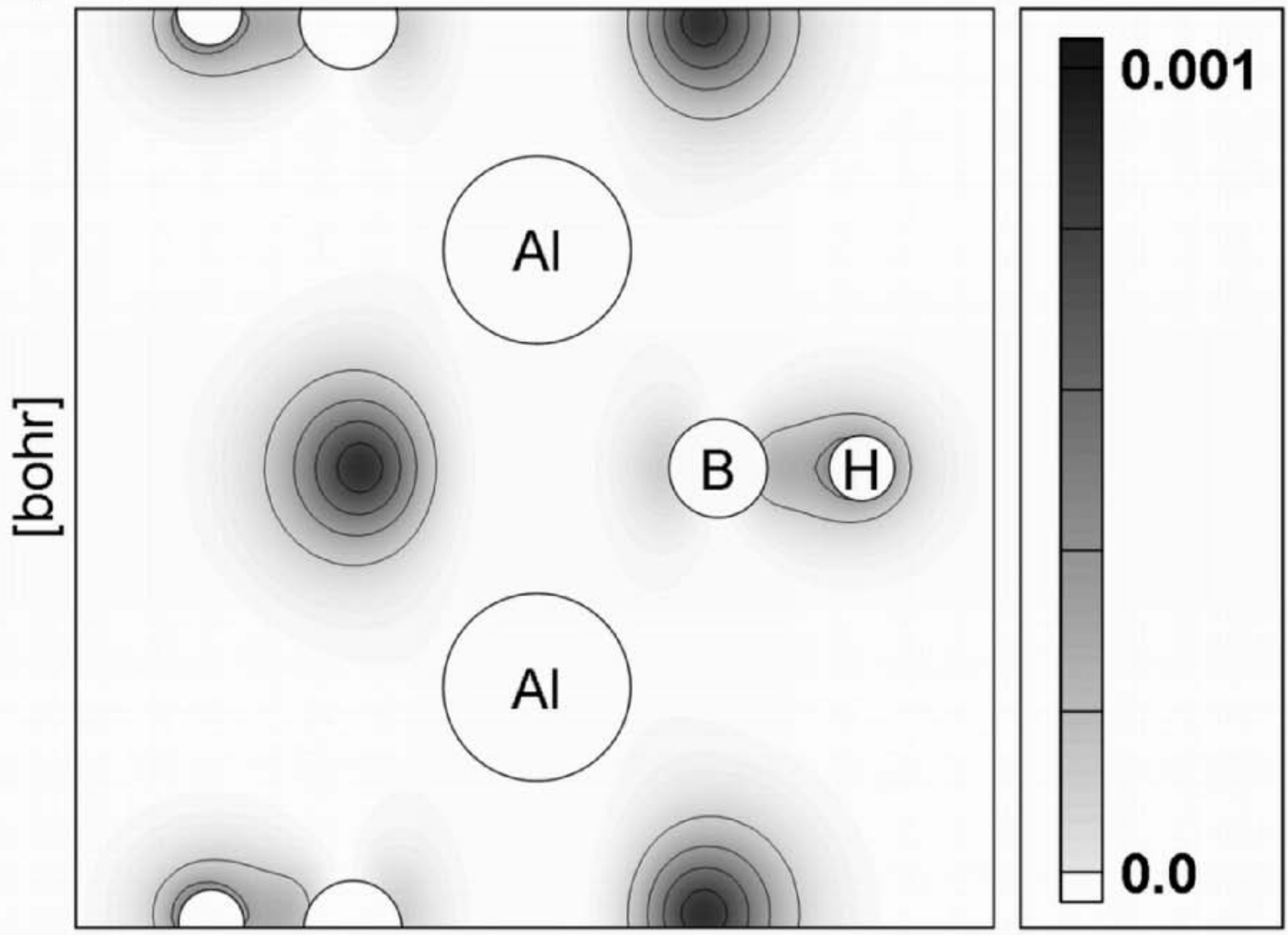

$(-7.00,0.00,-7.00)$

[bohr]

$(7.00,0.00,-7.00)$ 


\section{$(-7.00,7.00,0.00)$}

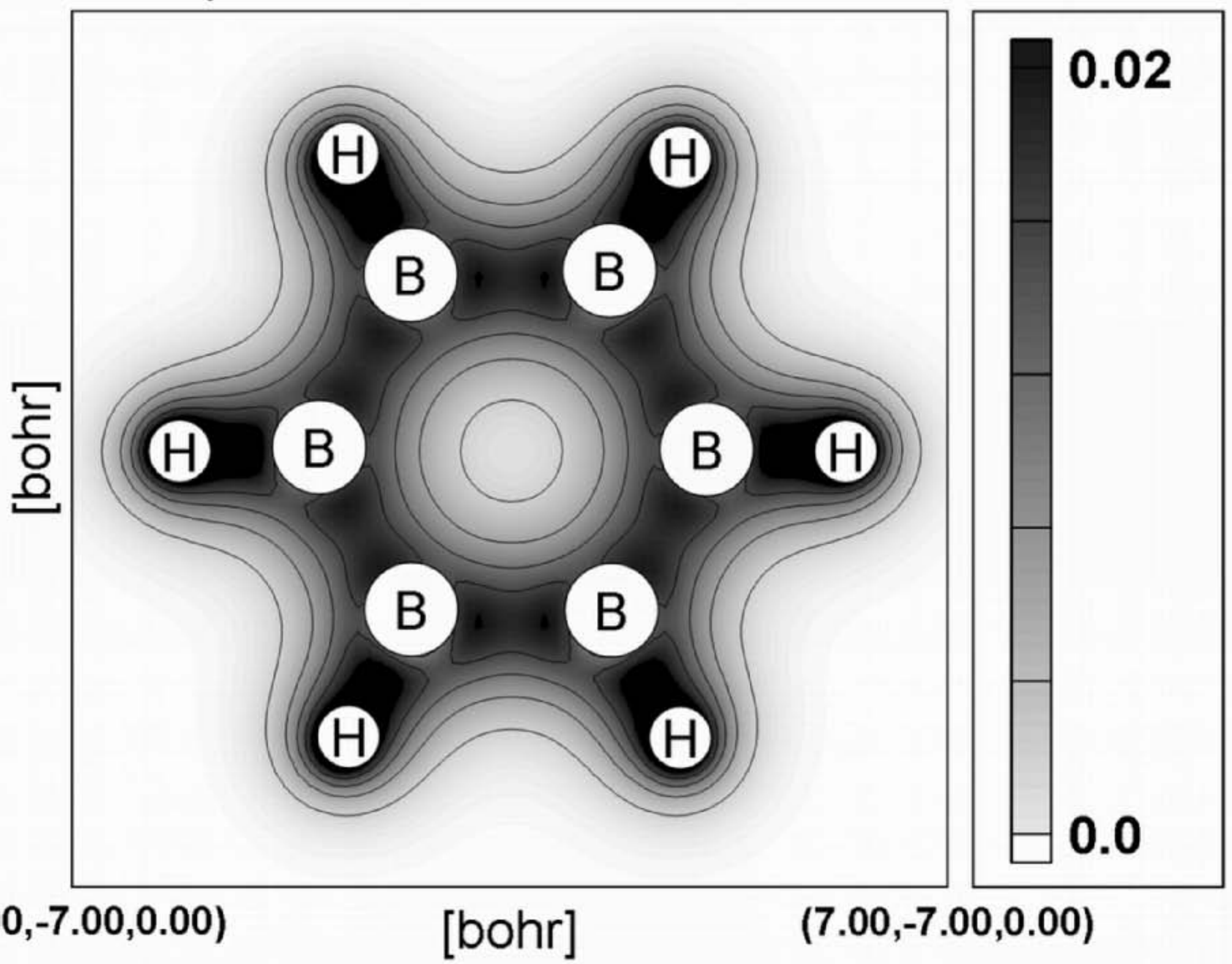

$(-7.00,-7.00,0.00)$

[bohr]

$(7.00,-7.00,0.00)$ 
$(-7.00,0.00,7.00)$

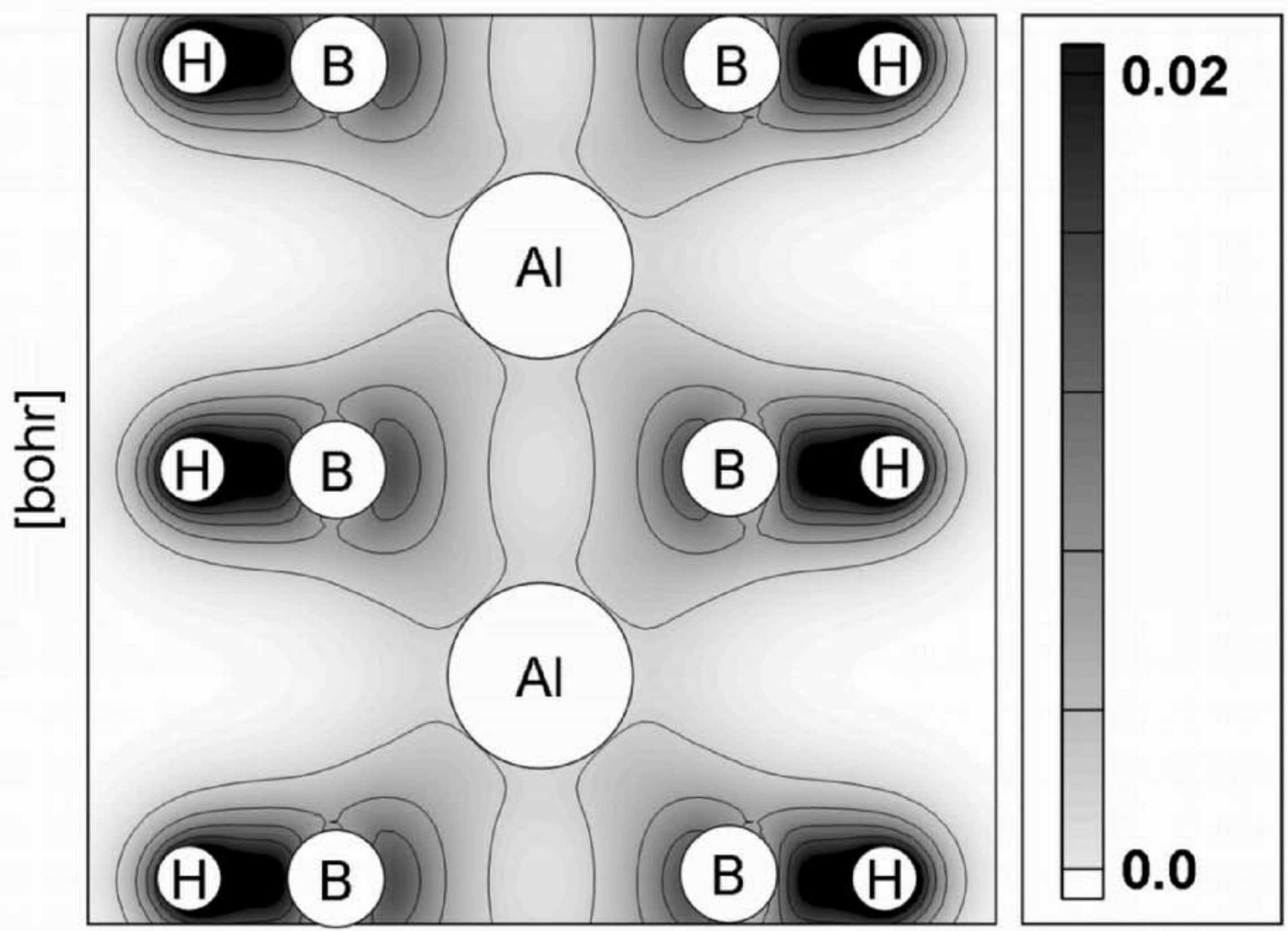

$(-7.00,0.00,-7.00)$

[bohr]

$(7.00,0.00,-7.00)$ 


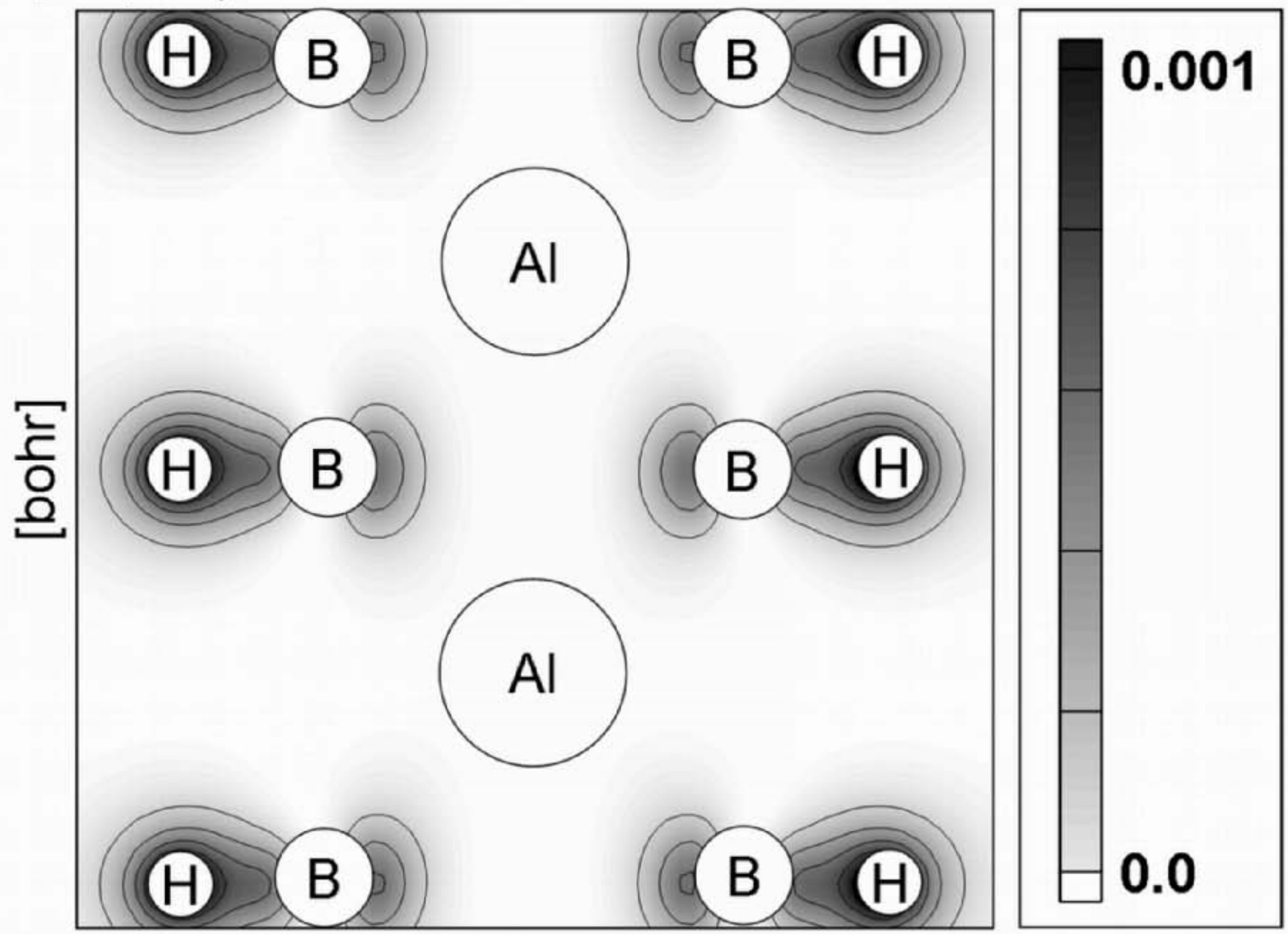




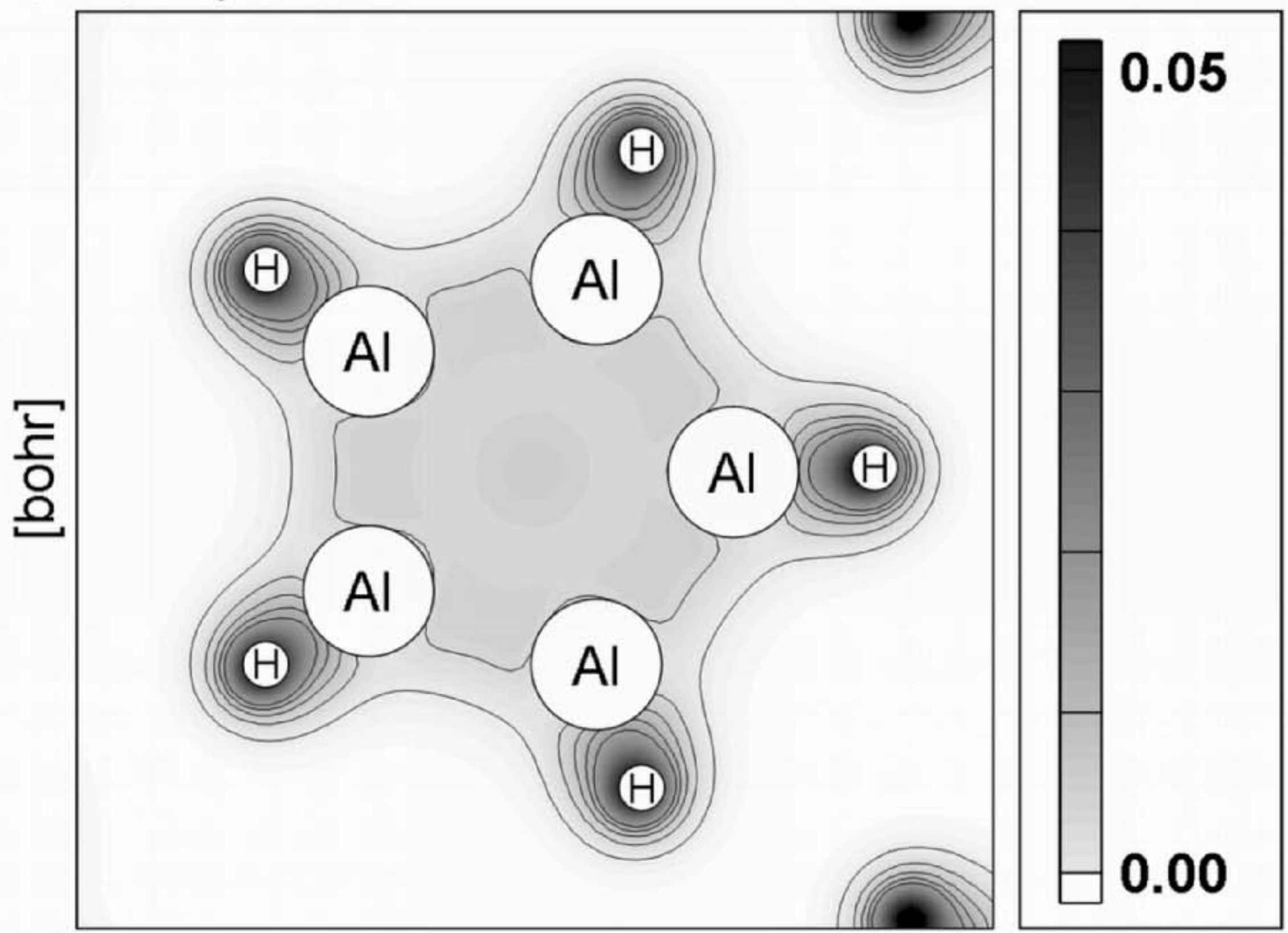




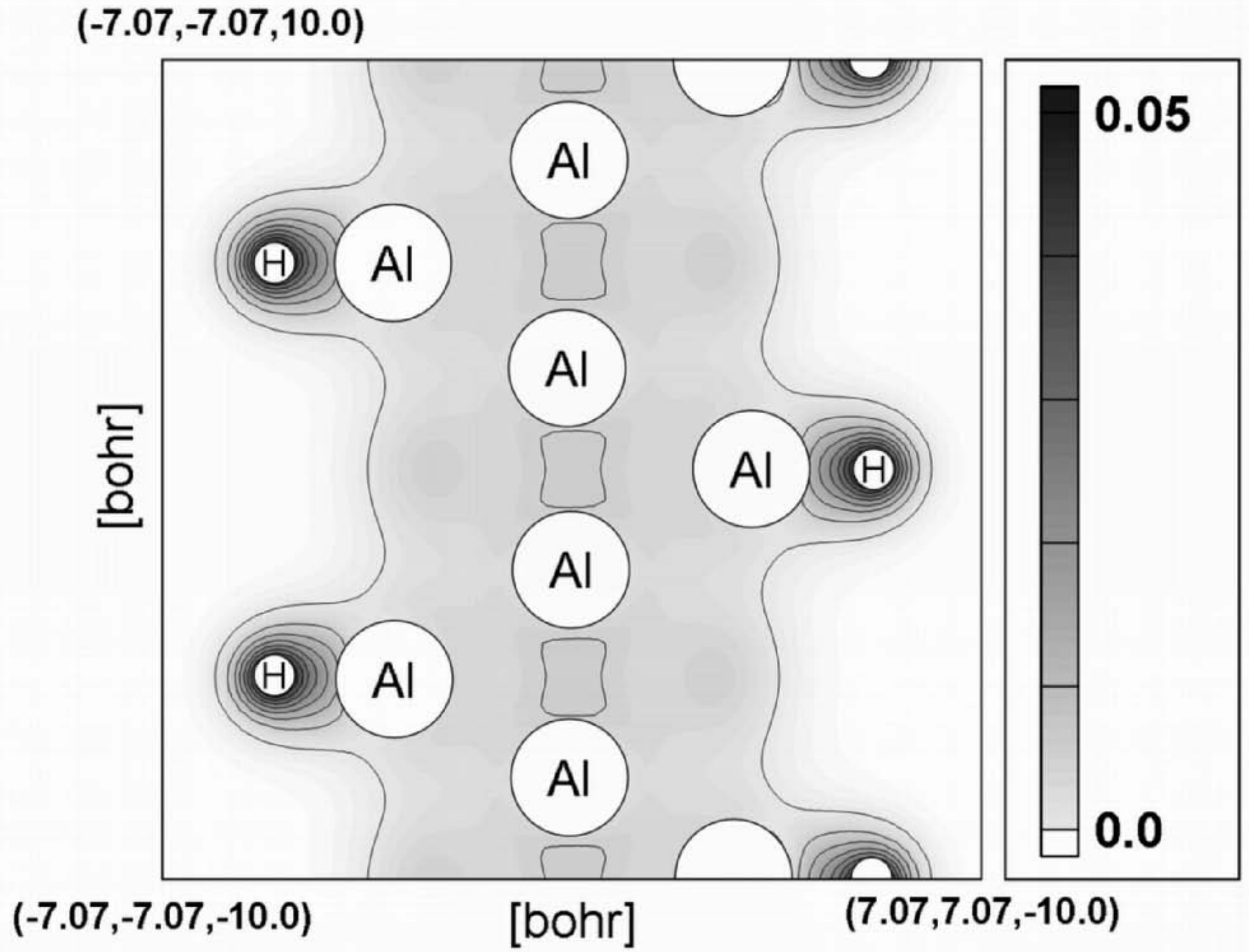




\section{$(-7.07,-7.07,10.0)$}

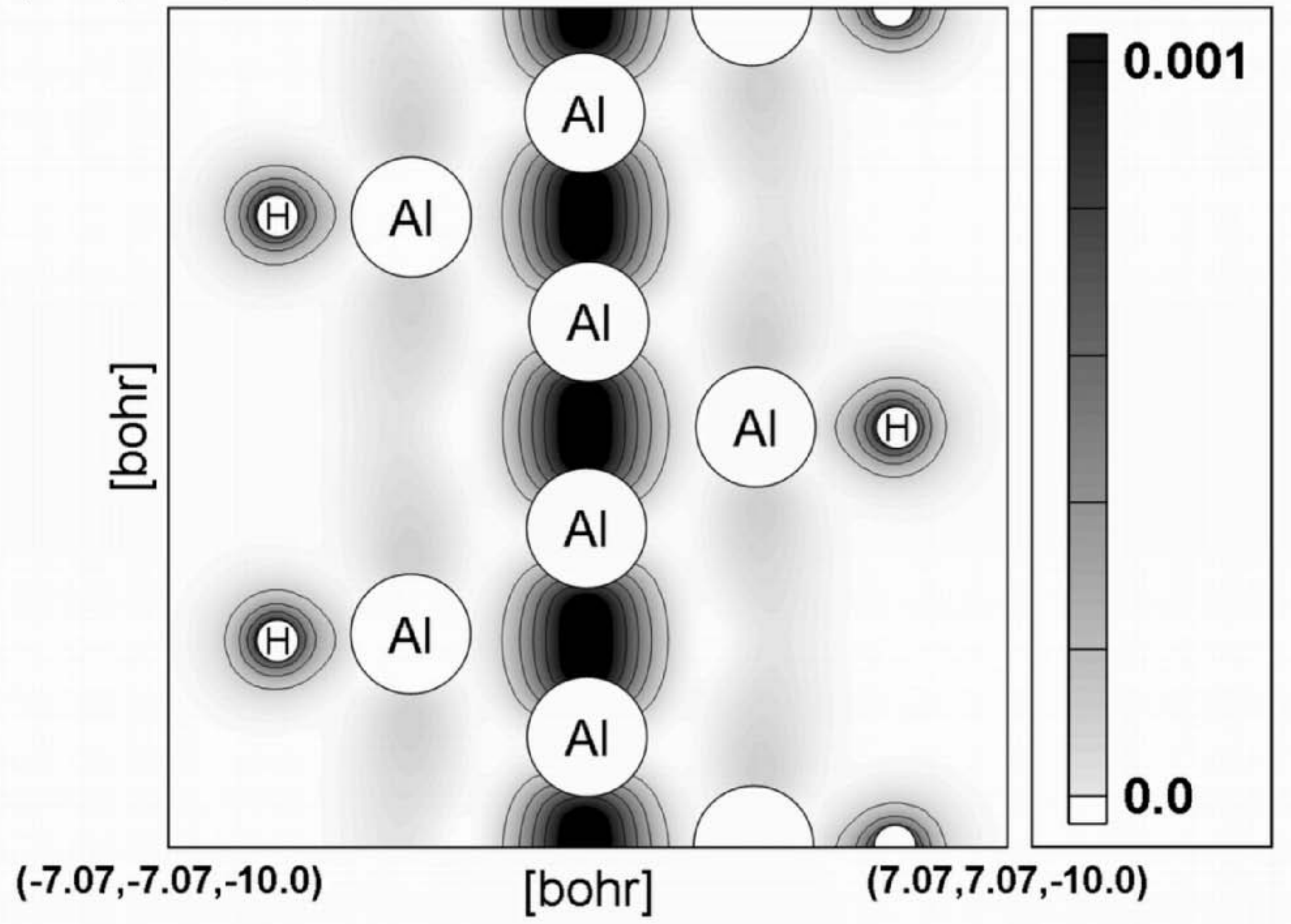



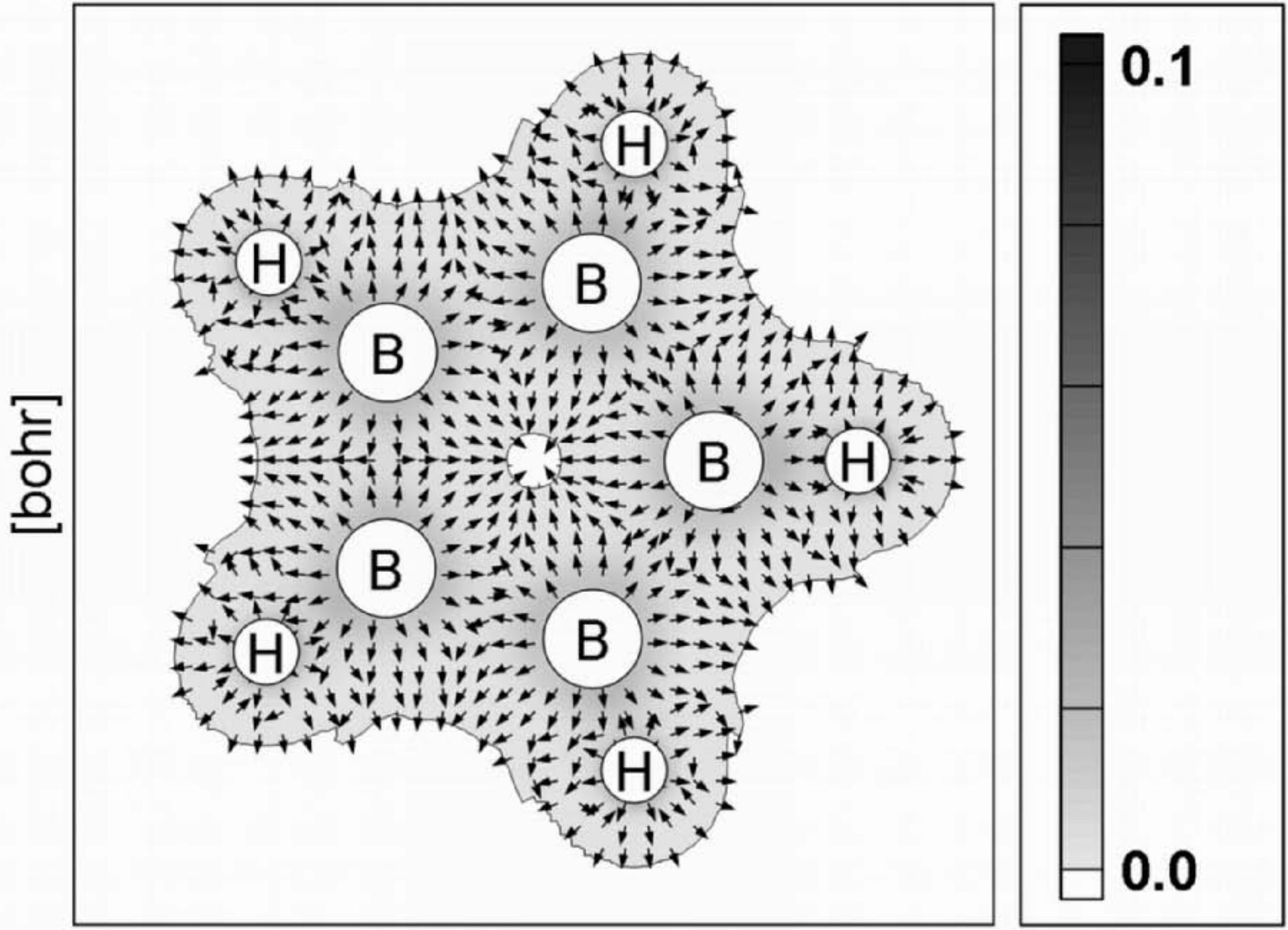

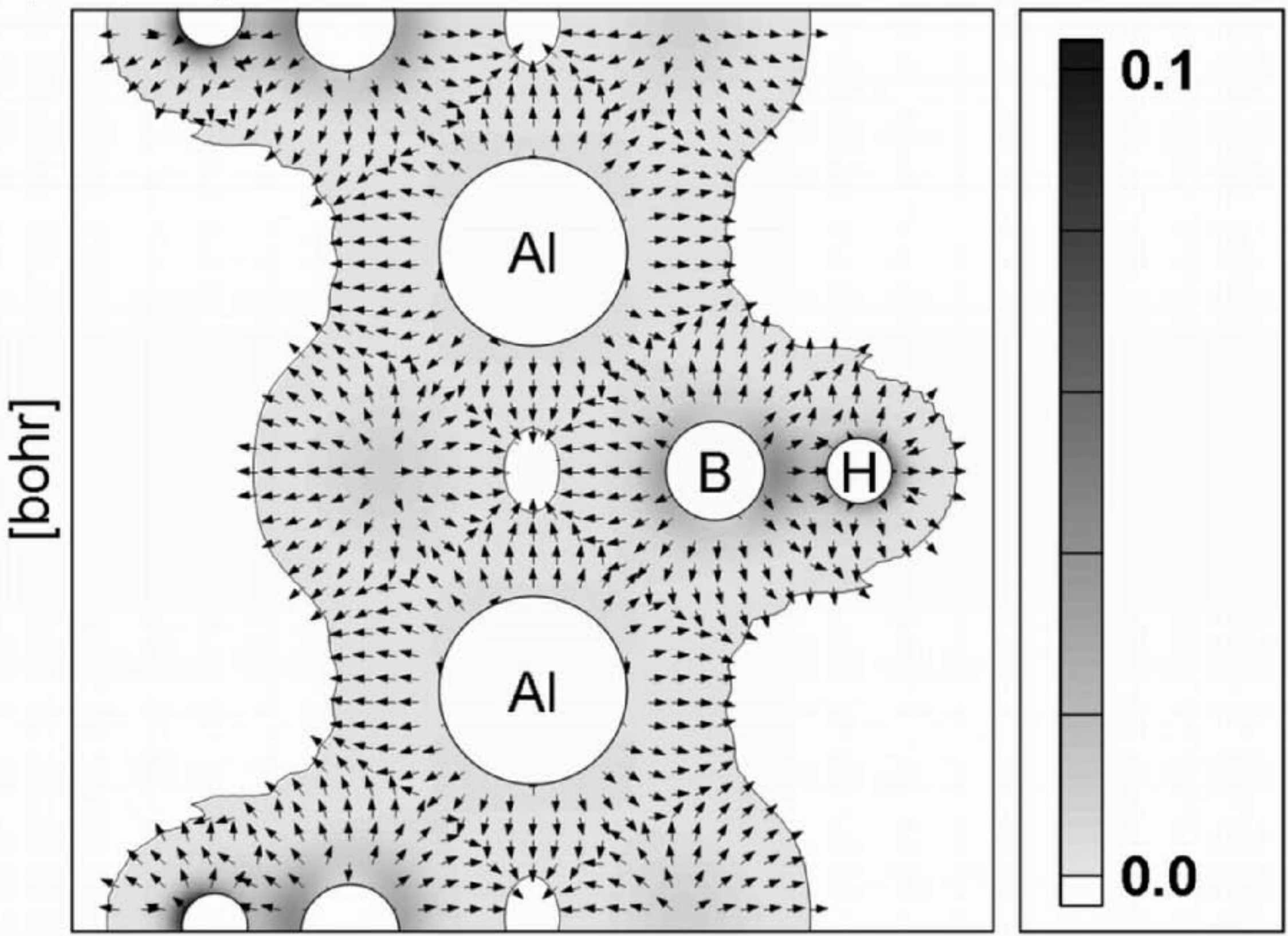

$(-7.00,0.00,-7.00)$

[bohr]

$(7.00,0.00,-7.00)$ 


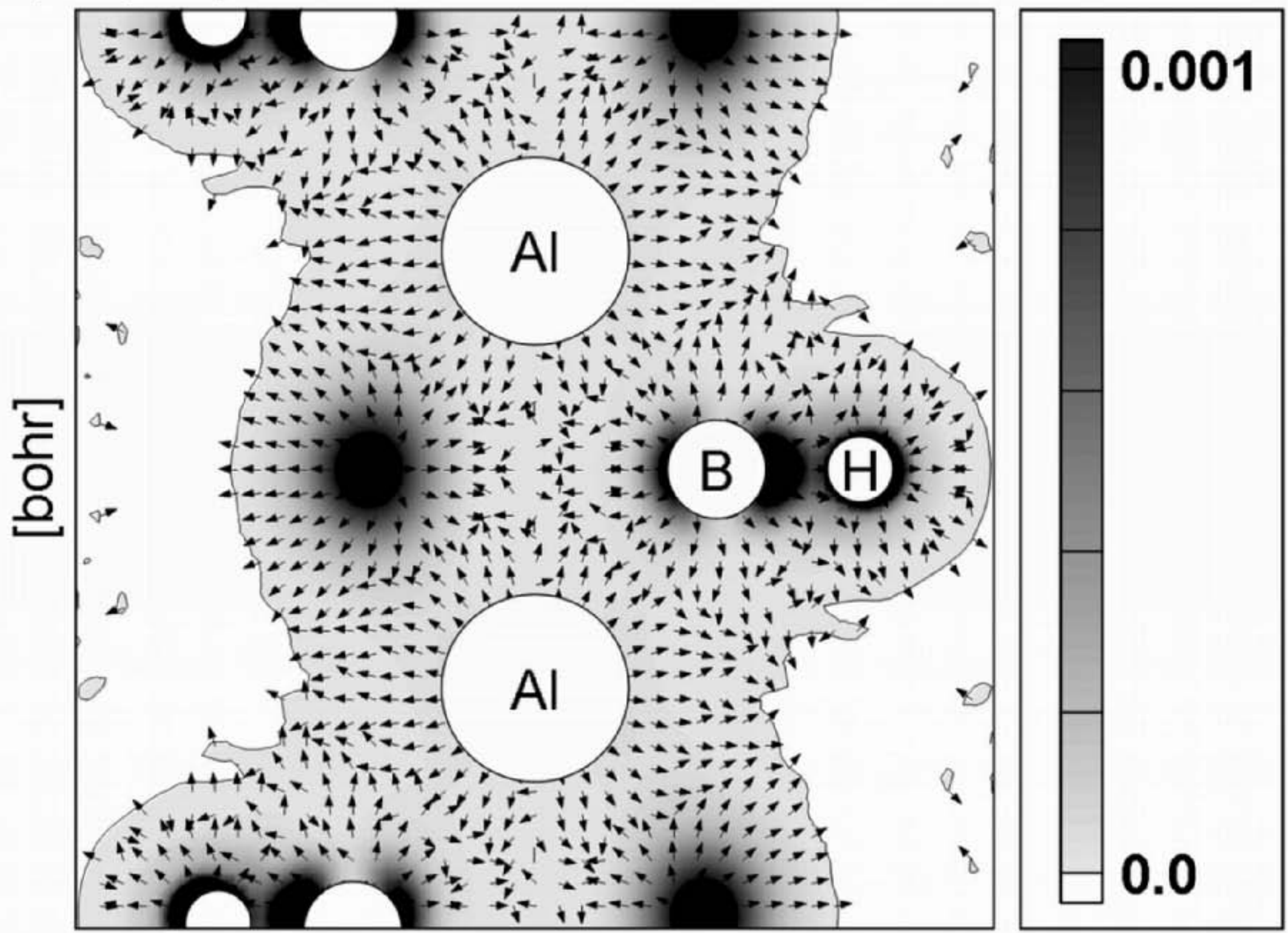

$(-7.00,0.00,-7.00)$

[bohr]

$(7.00,0.00,-7.00)$ 


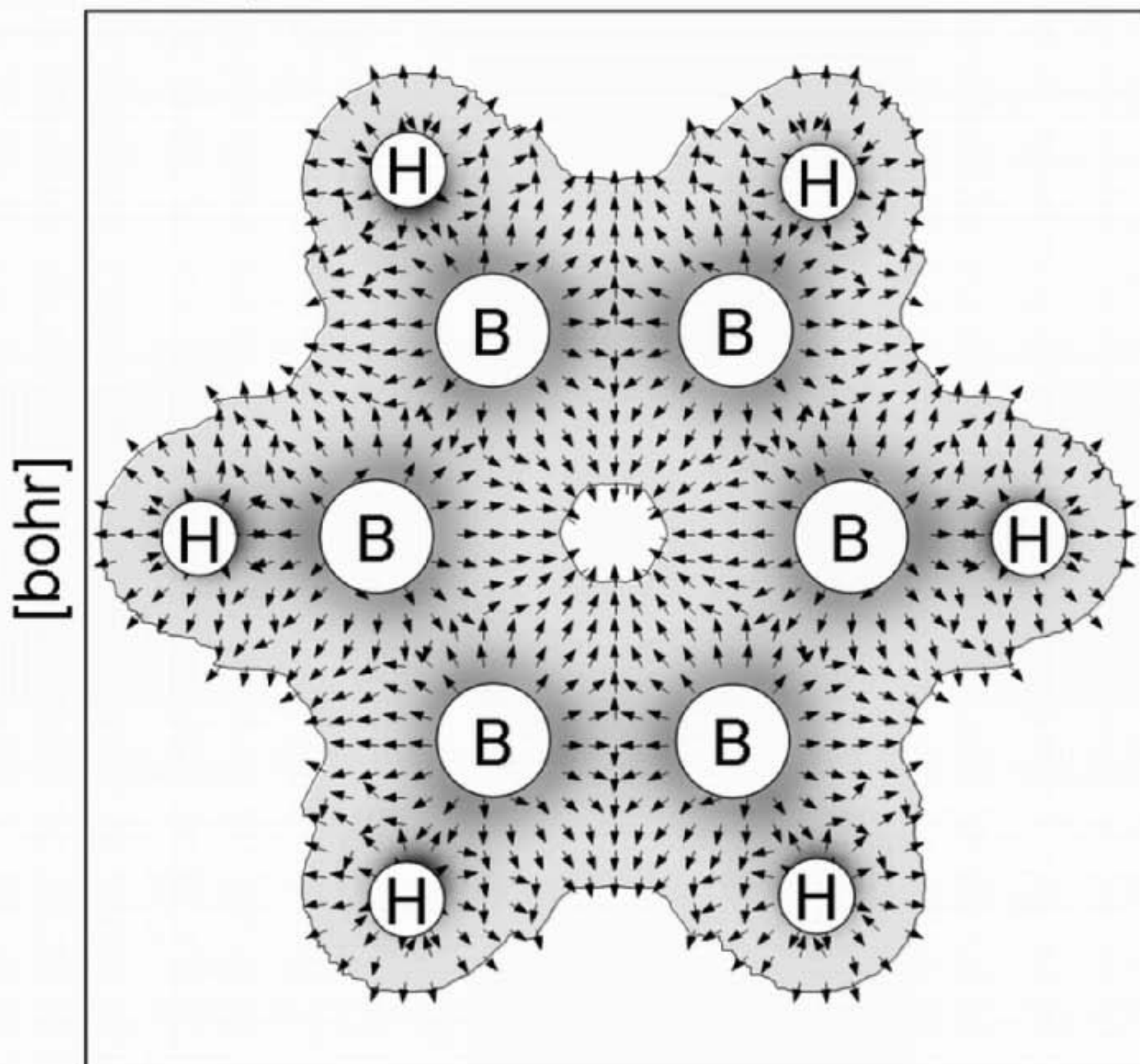

0.05

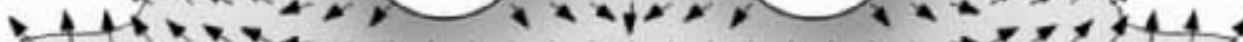

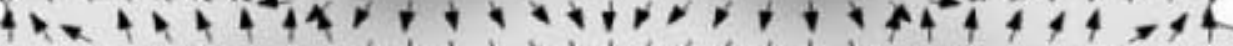

픙

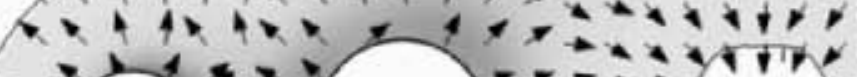

$7+4+4+4$

$B$

15: 
$(-7.00,0.00,7.00)$

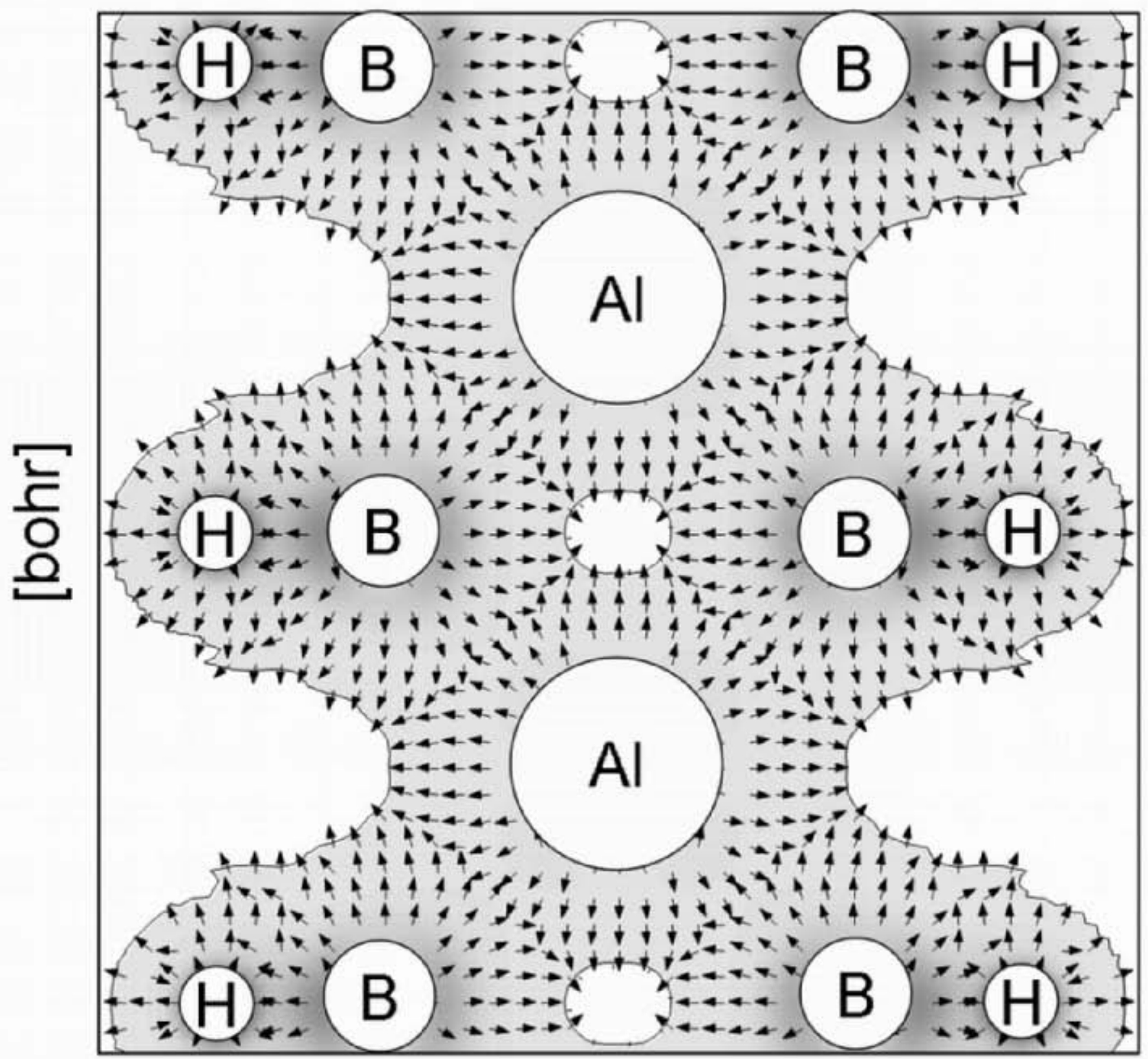

0.05

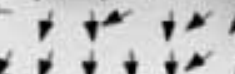

$4 x \rightarrow \rightarrow t^{4}+40+2$

$4+t+i+4$

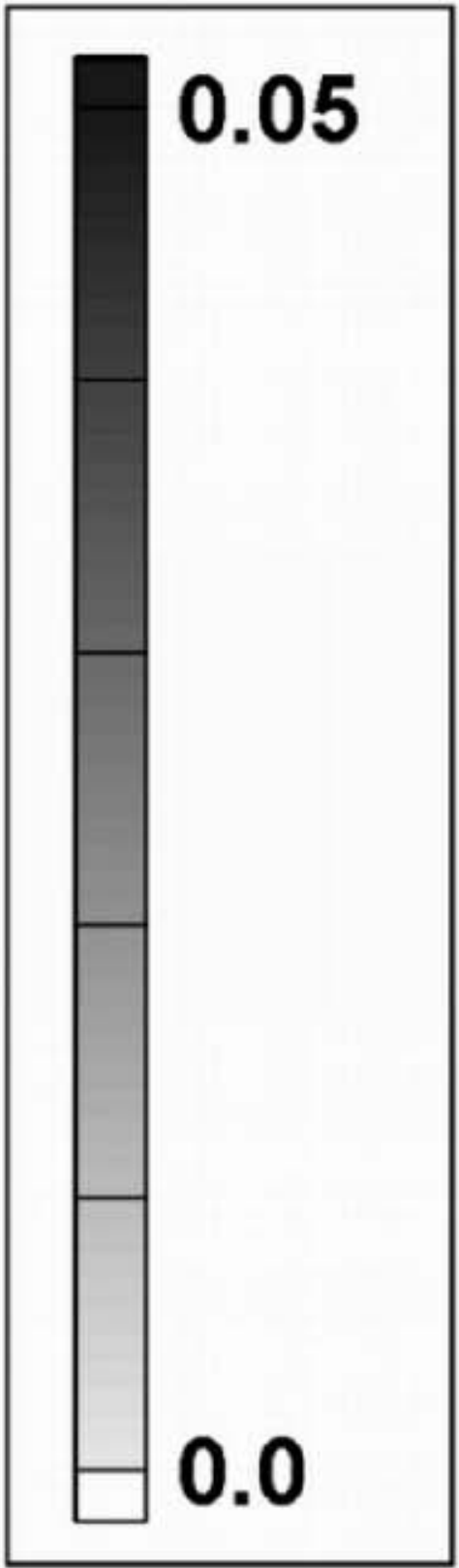

$(-7.00,0.00,-7.00)$

[bohr]

$(7.00,0.00,-7.00)$ 


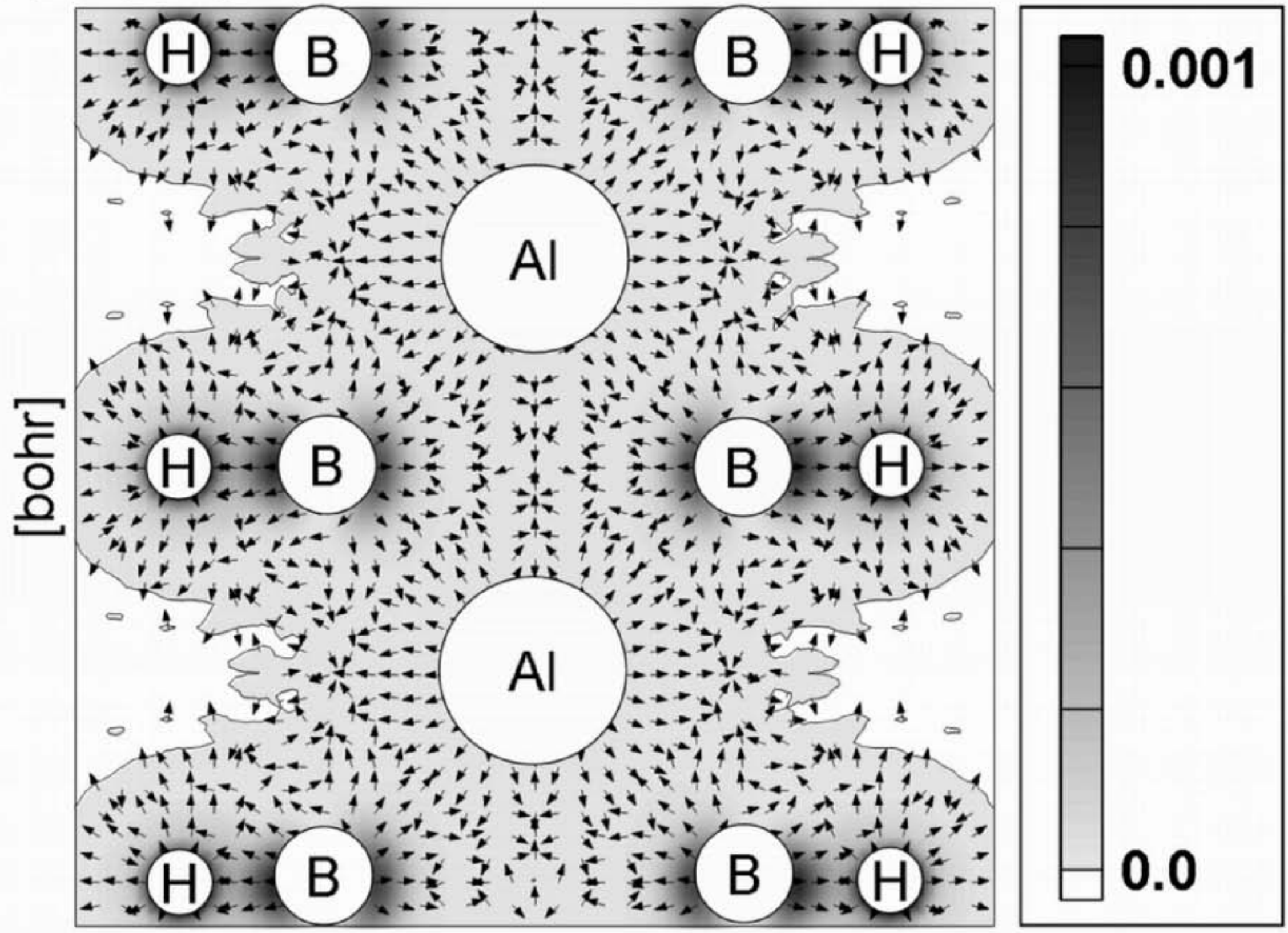




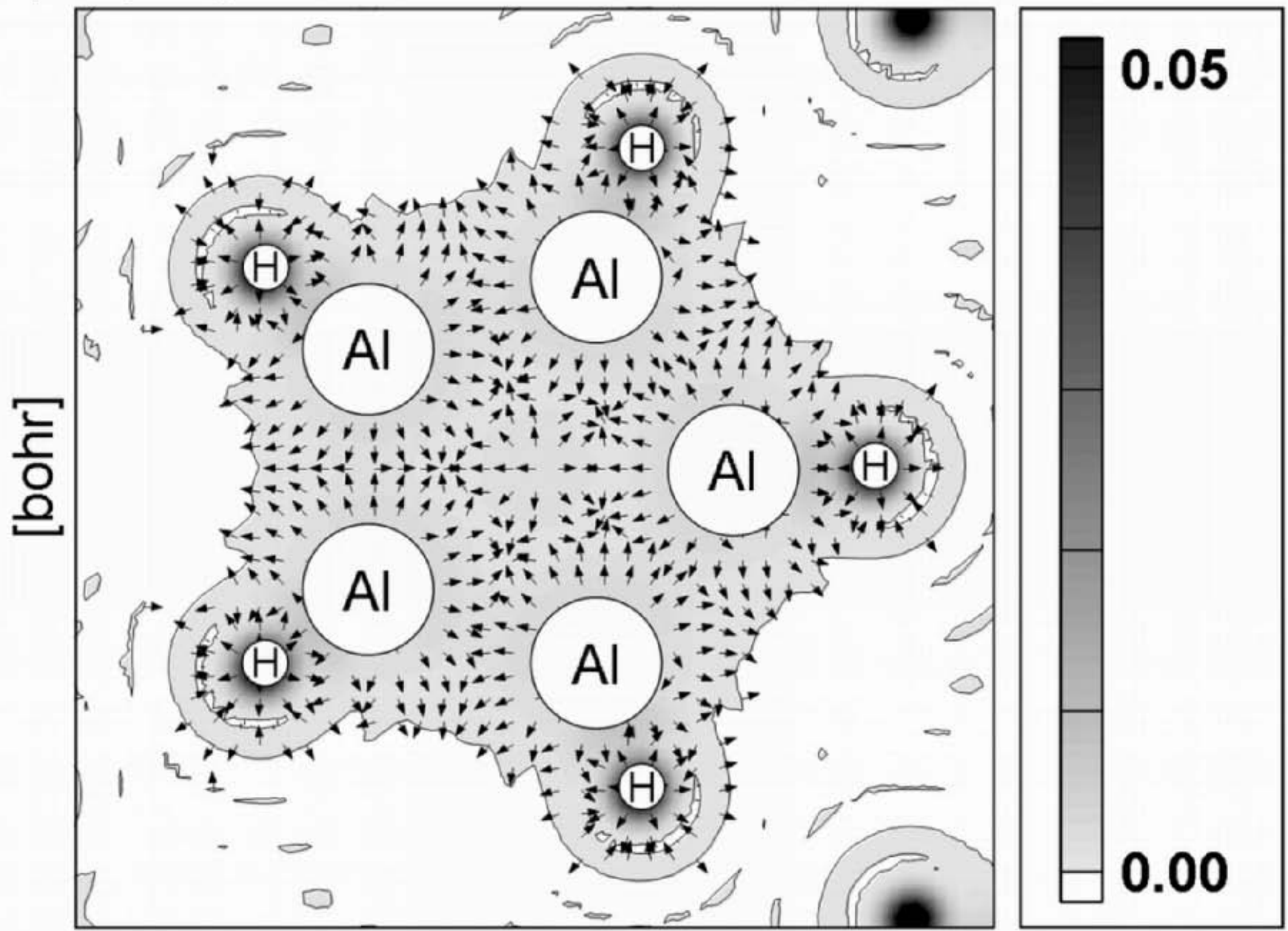

$(-7.07,-7.07,0.00)$

[bohr] 


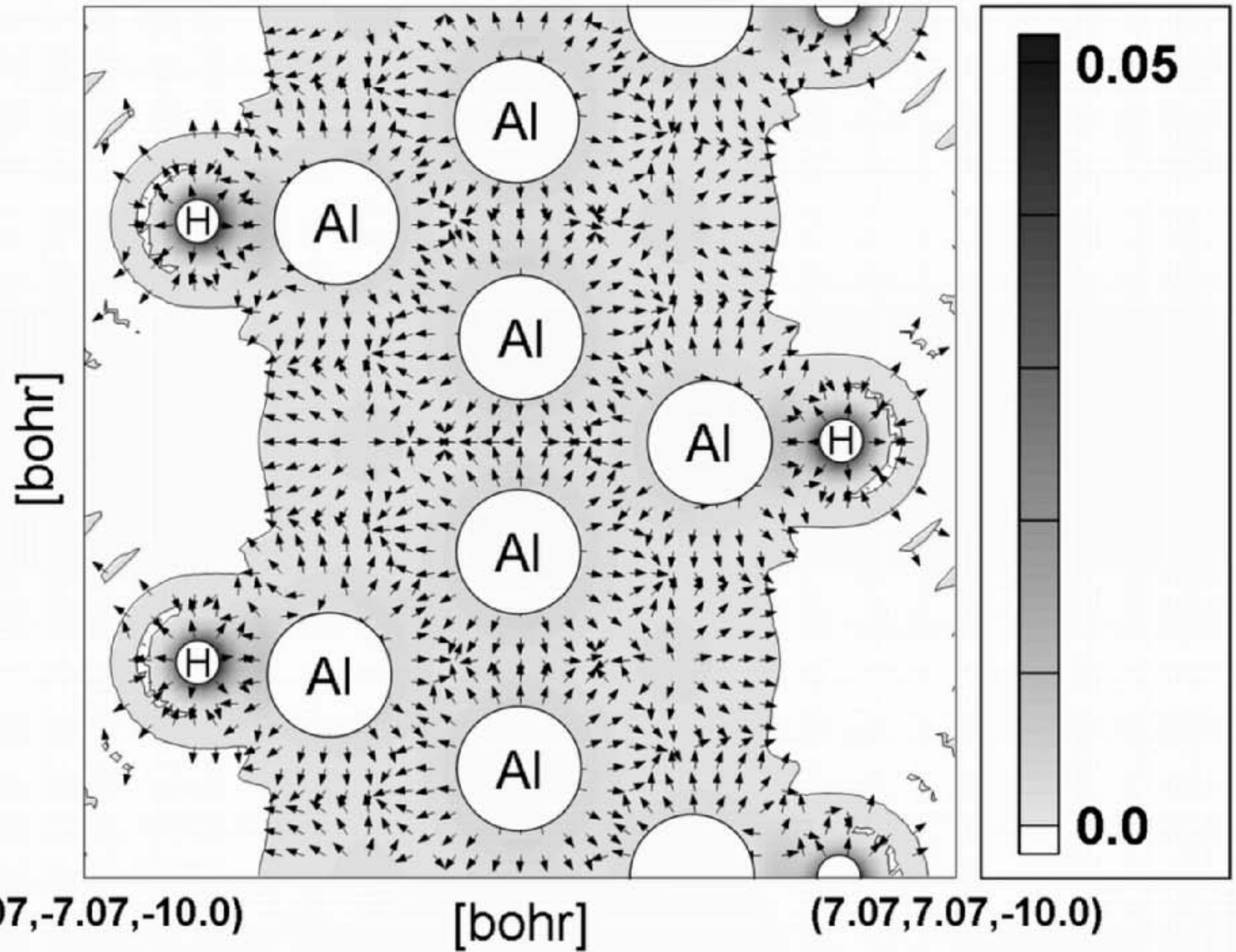

$(-7.07,-7.07,-10.0)$ 


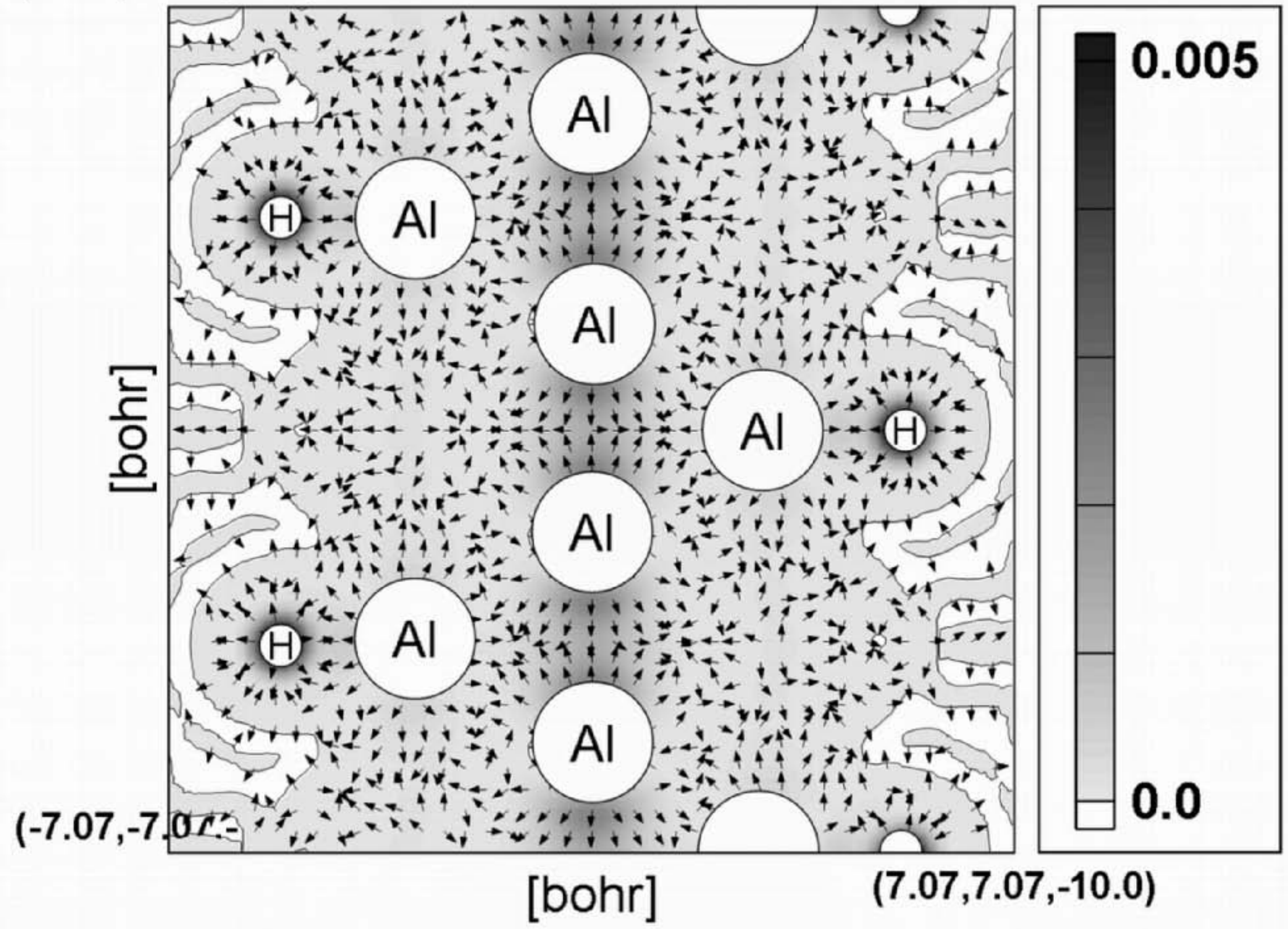




\section{$(-7.00,0.00,7.00)$}

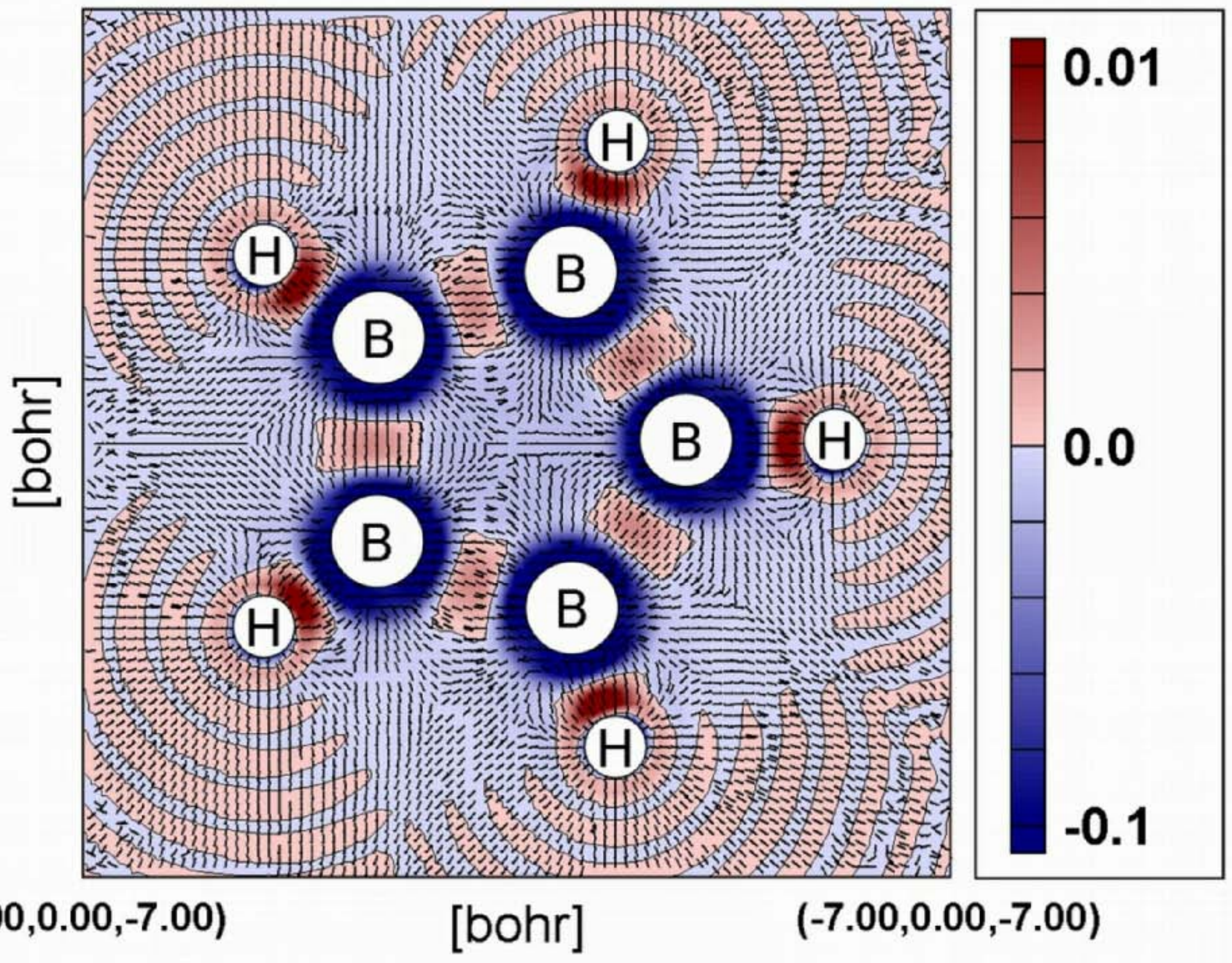

$(7.00,0.00,-7.00)$

[bohr]

$(-7.00,0.00,-7.00)$ 
$(-7.00,0.00,7.00)$

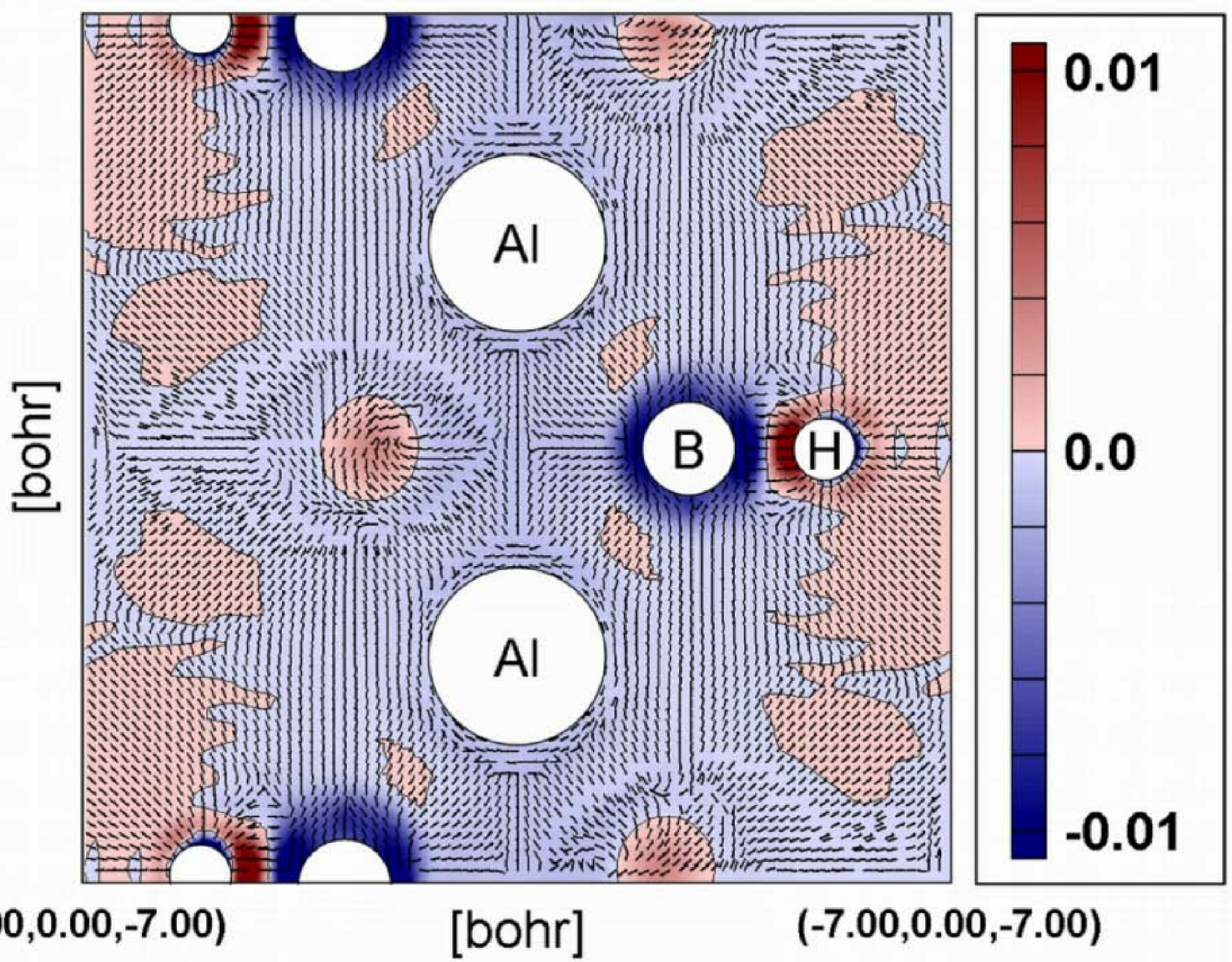

$(7.00,0.00,-7.00)$

[bohr]

$(-7.00,0.00,-7.00)$ 


\section{$(-7.00,0.00,7.00)$}

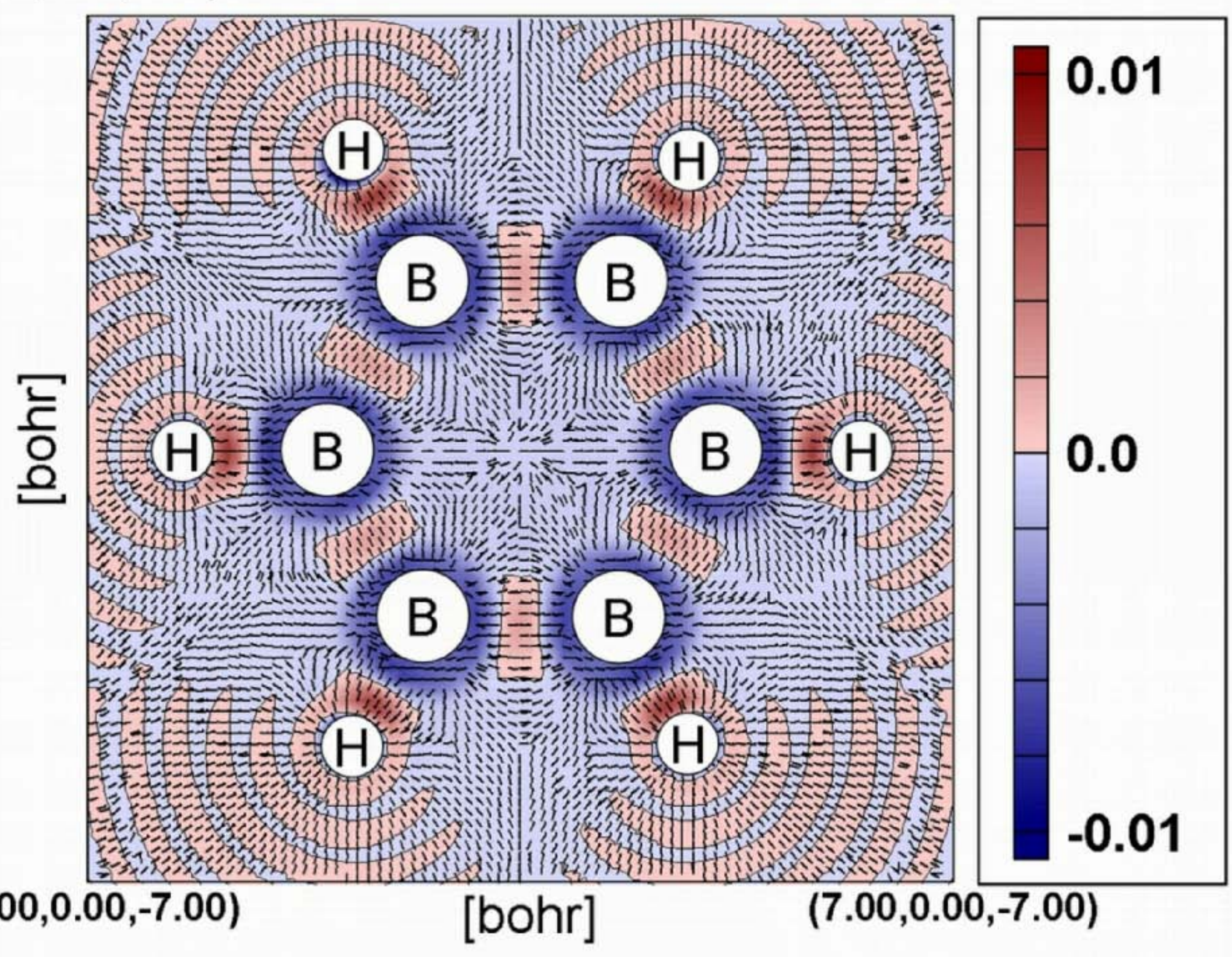



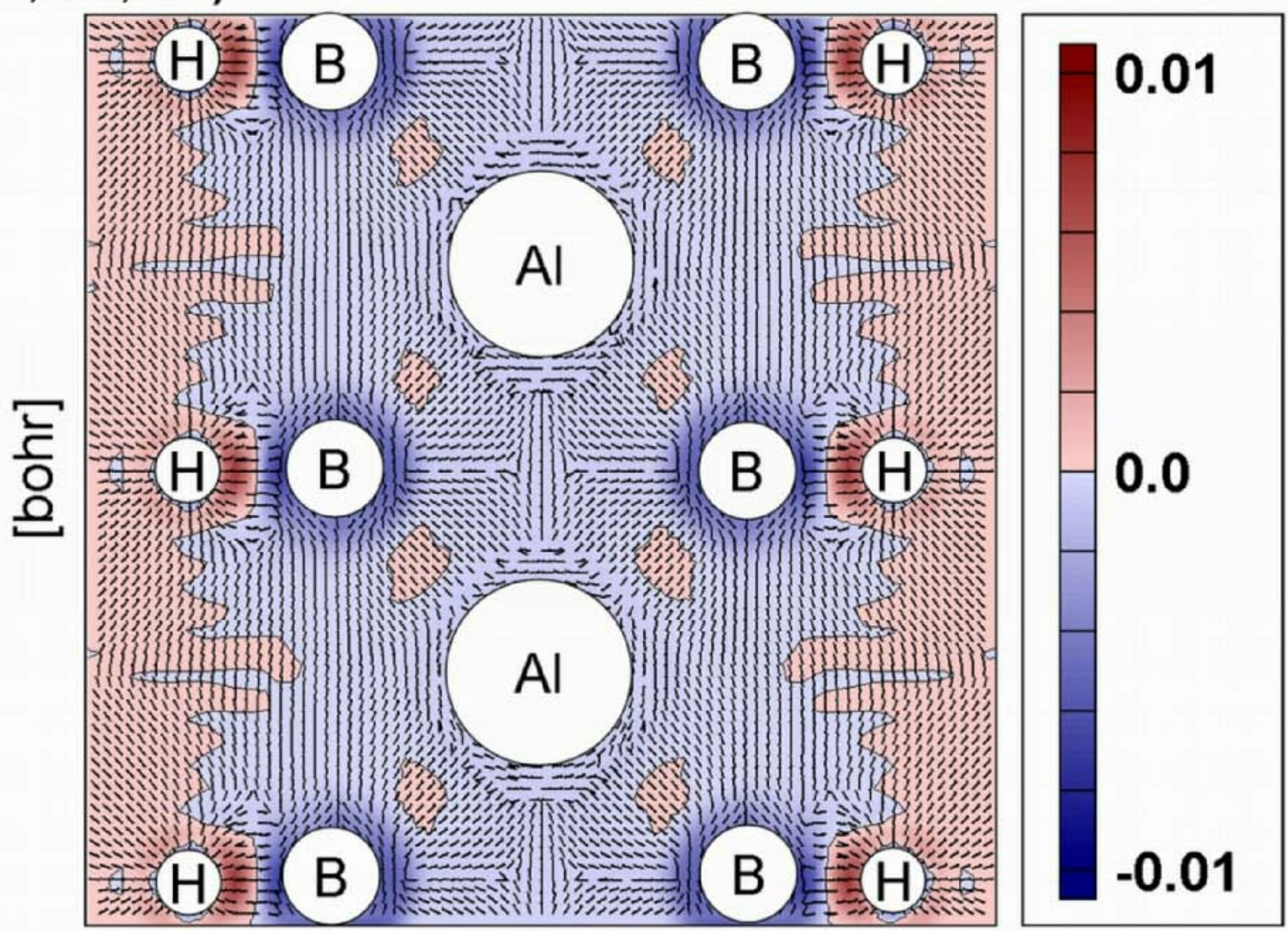

$(-7.00,0.00,-7.00)$

[bohr]

$(7.00,0.00,-7.00)$ 


\section{$(-7.07,-7.07,10.0)$}
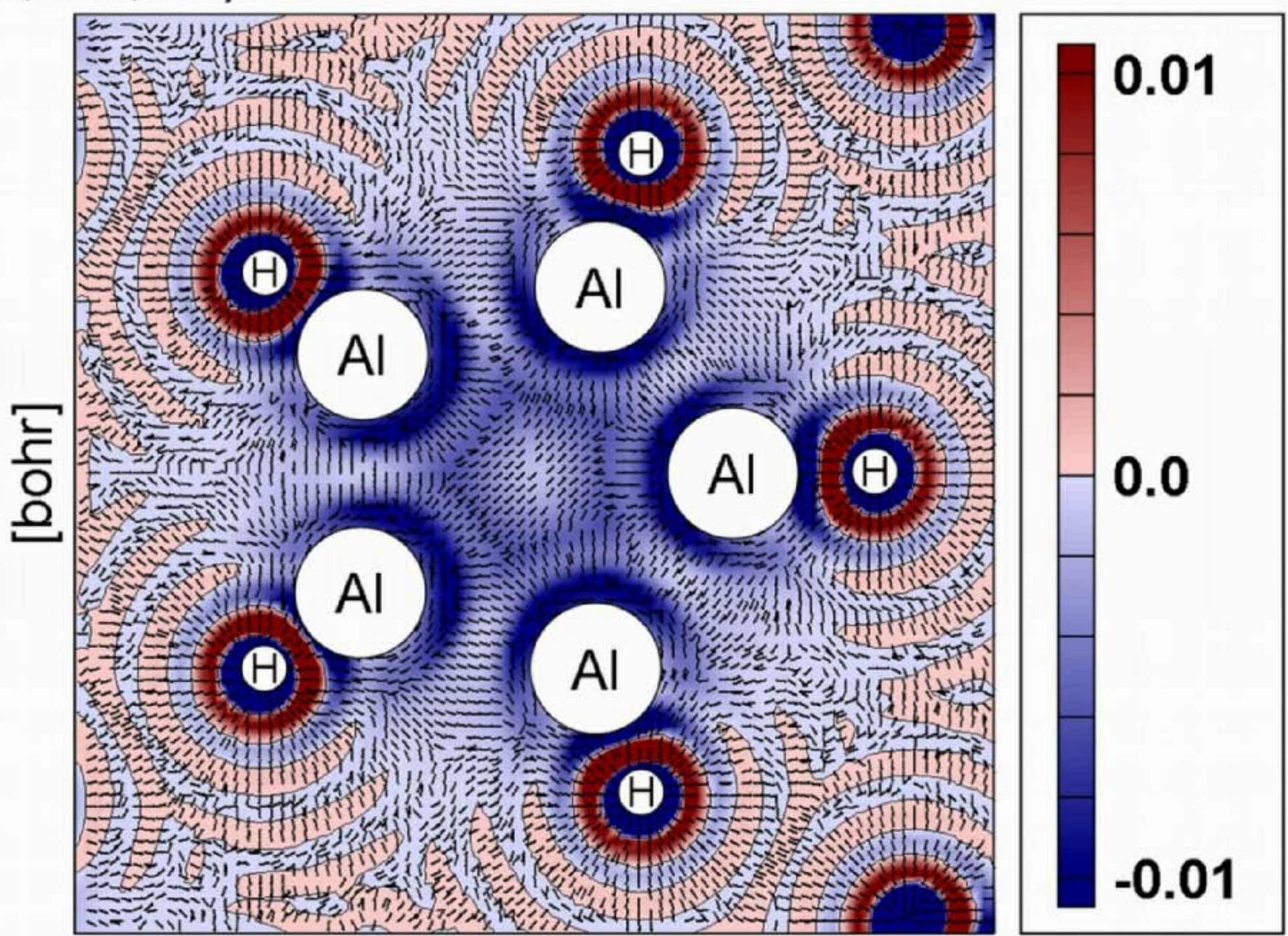

$(-7.07,-7.07,-10.0)$

[bohr]

$(7.07,7.07,-10.0)$ 

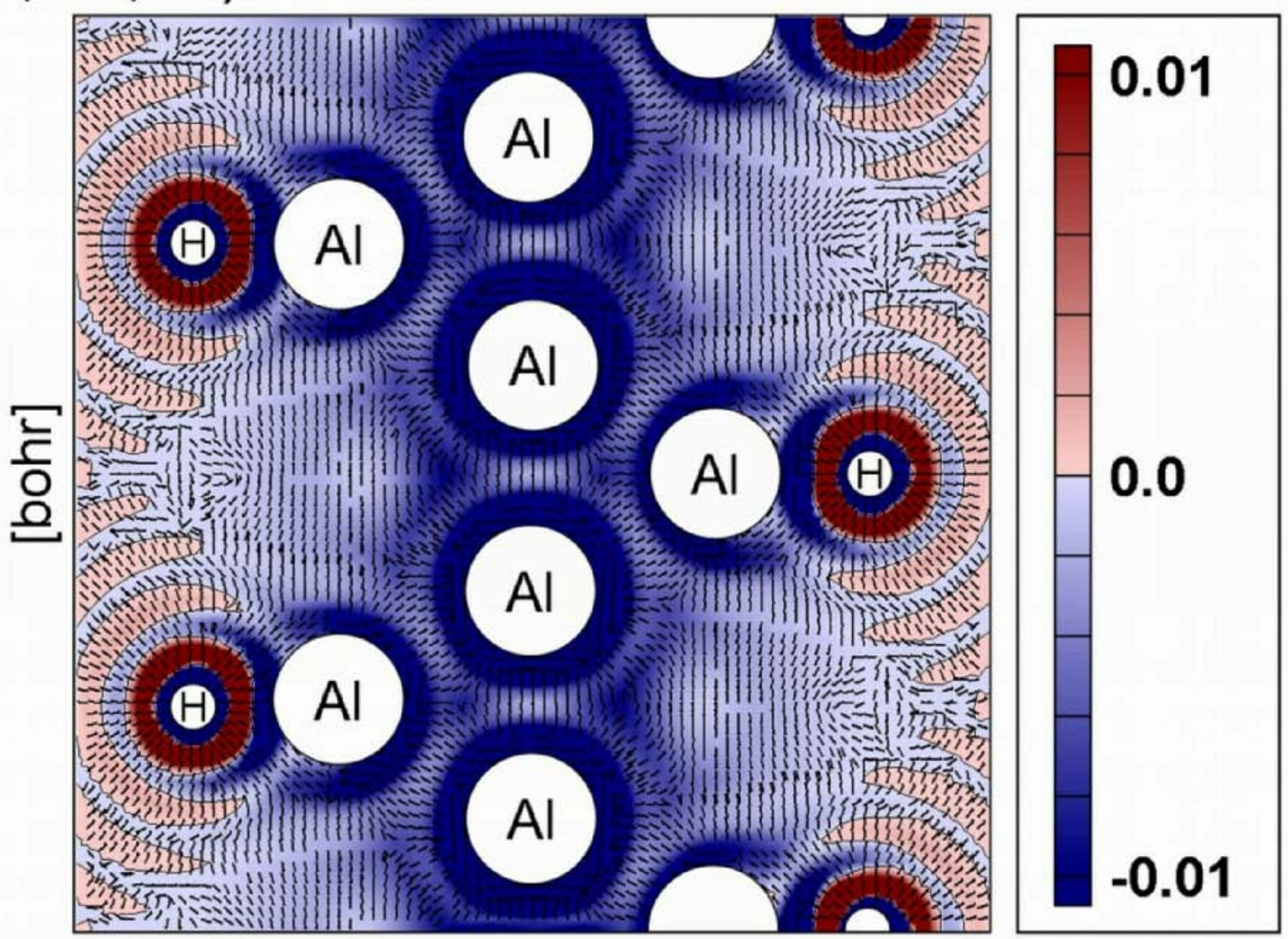

$(-7.07,-7.07,-10.0)$

[bohr]

$(7.07,7.07,-10.0)$ 